\title{
Nestable Tetrakis(spiroborate) Nanocycles
}

Supporting Information

Hiroshi Danjo, ${ }^{* \dagger}$ Yuhki Hashimoto ${ }^{\ddagger}{ }^{\ddagger}$ Yuki Kidena,${ }^{\ddagger}$ Ayumi Nogamine,${ }^{\ddagger}$ Kosuke Katagiri,${ }^{\dagger}$ Masatoshi Kawahata, ${ }^{\S}$ Toshifumi Miyazawa, ${ }^{\dagger}$ and Kentaro Yamaguchi ${ }^{\S}$

${ }^{\dagger}$ Department of Chemistry and ${ }^{\ddagger}$ Graduate School of Natural Science, Konan University, 8-9-1 Okamoto, Higashinada, Kobe 658-8501, Japan

${ }^{\S}$ Kagawa School of Pharmaceutical Sciences, Tokushima Bunri University, 1314-1 Shido, Sanuki, Kagawa 769-2193, Japan

\section{Table of Contents}

- General and Materials $\quad$ S2

- Synthesis $\quad$ S3

- Spectral copies of ${ }^{1} \mathrm{H}$ and ${ }^{13} \mathrm{C}$ NMR $\quad$ S12

- Experimental details of Figures 1,2,4, and $5 \quad$ S27

- ${ }^{1} \mathrm{H}$ NMR spectra of mixtures of $\mathbf{4 b} \cdot\left(\mathrm{Me}_{2} \mathrm{NH}_{2}\right)_{4}, \mathbf{7}$, and $\mathbf{8} \cdot\left(\mathrm{PF}_{6}\right)_{2}$ (Figure S1) S29

- ${ }^{1} \mathrm{H}$ NMR spectra of mixtures of $\mathbf{8} \cdot\left(\mathrm{PF}_{6}\right)_{2}$ and 9·(TBA) $)_{2}$ (Figure S2) S30

- Job plot of $\mathbf{4 b} \cdot\left(\mathrm{Me}_{2} \mathrm{NH}_{2}\right)_{4}$ and $\mathbf{8} \cdot\left(\mathrm{PF}_{6}\right)_{2}$ (Figure S3) S31

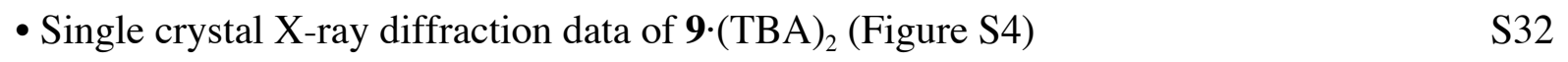

- Single crystal X-ray diffraction data of $[\mathbf{1 1} @ \mathbf{1 0}] \cdot\left(\mathrm{PF}_{6}\right)_{4}$ (Figure S5) S33

- ${ }^{1} \mathrm{H}-{ }^{1} \mathrm{H}$ NOESY spectrum of 11@10@5b (Figure S6) S34

- CSI-Mass spectra of a mixture of $\mathbf{5 b} \cdot\left(\mathrm{Me}_{2} \mathrm{NH}_{2}\right)_{4}, \mathbf{1 0} \cdot\left(\mathrm{PF}_{6}\right)_{4}$, and $\mathbf{1 1}$ (Figure S7) S35

- Single crystal X-ray diffraction data of 10@5b (Figure S8) S36

- References $\quad$ S37 
General. All manipulations were carried out under nitrogen atmosphere. NMR spectra were recorded on a Varian UNITY INOVA $500\left(500 \mathrm{MHz}\right.$ for ${ }^{1} \mathrm{H}$, and $126 \mathrm{MHz}$ for $\left.{ }^{13} \mathrm{C}\right)$. Chemical shifts were reported in $\delta \mathrm{ppm}$ referenced to an internal tetramethylsilane standard for ${ }^{1} \mathrm{H} \mathrm{NMR}$. Residual chloroform ( $\delta 77.0 \mathrm{ppm}$ for ${ }^{13} \mathrm{C}$ ) was used as internal reference for ${ }^{13} \mathrm{C} \mathrm{NMR} .{ }^{1} \mathrm{H}$ and ${ }^{13} \mathrm{C}$ NMR spectra were recorded in $\mathrm{CDCl}_{3}$ at $25{ }^{\circ} \mathrm{C}$ unless otherwise noted. IR spectra were recorded on a JASCO FT/IR-4200. Optical rotations were recorded on a JASCO DIP-1000 polarimeter with a sodium lamp. Nominal (LRMS) spectra were recorded on a JEOL JMS-600H or a JEOL JMS-T100LP, and exact mass (HRMS) spectra were recorded on a Bruker FT-ICR apex-Qe or a JEOL JMS-T100LP. X-ray diffraction images of the crystals were collected using a Rayonix MarMosaic225 CCD or a Rayonix MX225HE CCD area detector with synchrotron radiation at a wavelength of $0.7 \AA$ or $0.8 \AA$ at the BL26B2 or BL38B1 station of SPring-8 (Hyogo, Japan). The distance between the crystal and the detector was 55 or $85 \mathrm{~mm}$. Images were processed using software HKL2000 (HKL Research).

Materials. All reagents were obtained from commercial sources and used without further purification. Compound $\mathbf{1 a},{ }^{1)} 3{ }^{2)} \mathbf{8} \cdot\left(\mathrm{PF}_{6}\right)_{4},{ }^{3)} \mathbf{1 0} \cdot\left(\mathrm{PF}_{6}\right)_{4}{ }^{4)}$ and $\mathbf{1 7 \mathbf { a } ^ { 5 } )}$ were prepared according to the literature procedure. 


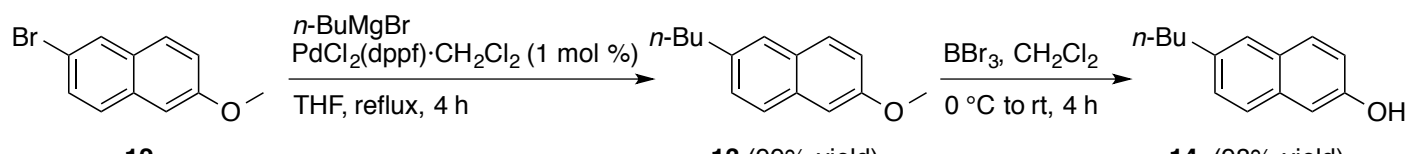

12

$13(99 \%$ yield $)$

14 (98\% yield)
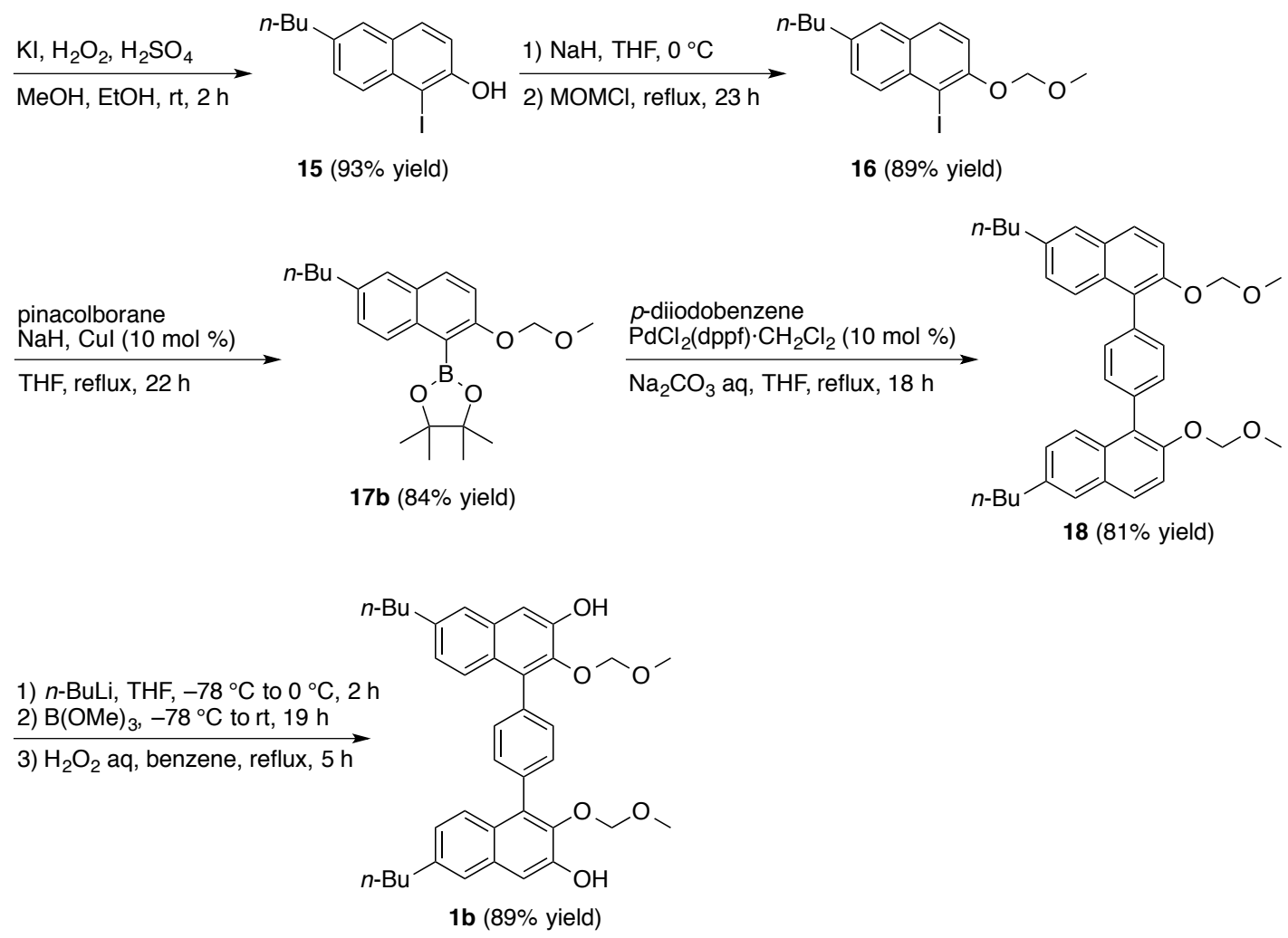

\section{Preparation of $13^{6,7)}$}

An oven-dried $300 \mathrm{~mL}$ 2-necked flask equipped with a condenser was charged with 7 (2.37 g, $10.0 \mathrm{mmol}), \mathrm{PdCl}_{2}(\mathrm{dppf}) \cdot \mathrm{CH}_{2} \mathrm{Cl}_{2}(82.0 \mathrm{mg}, 0.10 \mathrm{mmol})$, and $\mathrm{THF}(104 \mathrm{~mL})$ at nitrogen atmosphere, and the solution was cooled to $0{ }^{\circ} \mathrm{C}$. To this solution was added $n$-Bu-MgBr (19.4 mL of $1.03 \mathrm{M}$ THF solution, $20.0 \mathrm{mmol}$ ) dropwise at $0{ }^{\circ} \mathrm{C}$, and the mixture was refluxed for $4 \mathrm{~h}$. After allowed to room temperature, the reaction was quenched with sat. $\mathrm{NH}_{4} \mathrm{Cl}$ aq., and extracted with EtOAc. The organic layer was washed with sat. $\mathrm{NH}_{4} \mathrm{Cl}$ aq., sat. $\mathrm{NaHCO}_{3}$ aq., brine, and dried over $\mathrm{Na}_{2} \mathrm{SO}_{4}$. After evaporation, the residue was subjected to column chromatography on silica gel $(n$-hexane/EtOAc $=30 / 1)$ to give $\mathbf{1 3}$ as a white solid (2.13 g, 99\% yield); ${ }^{1} \mathrm{H}$ NMR (500 MHz, $\left.\mathrm{CDCl}_{3}\right) \delta(\mathrm{ppm}) 7.67(\mathrm{~d}, J=9.5 \mathrm{~Hz}, 1 \mathrm{H}), 7.66(\mathrm{~d}, J$ $=8.0 \mathrm{~Hz}, 1 \mathrm{H}), 7.53(\mathrm{~s}, 1 \mathrm{H}), 7.29(\mathrm{dd}, J=8.5,1.5 \mathrm{~Hz}, 1 \mathrm{H}), 7.11(\mathrm{dd}, J=8.0,3.0 \mathrm{~Hz}, 1 \mathrm{H}), 7.10$ $(\mathrm{s}, 1 \mathrm{H}), 3.91(\mathrm{~s}, 3 \mathrm{H}), 2.74(\mathrm{t}, J=7.5 \mathrm{~Hz}, 2 \mathrm{H}), 1.69-1.63(\mathrm{~m}, 2 \mathrm{H}), 1.39$ (sextet, $J=7.5 \mathrm{~Hz}$, $2 \mathrm{H}) ; 0.94(\mathrm{t}, J=7.5 \mathrm{~Hz}, 3 \mathrm{H})$; HRMS (ESI+): $\mathrm{m} / z$ Calcd. for $\mathrm{C}_{15} \mathrm{H}_{19} \mathrm{O}[\mathrm{M}+\mathrm{H}]^{+}: 215.14304$ Found: 215.14312.

\section{Preparation of 14}

An oven-dried $200 \mathrm{~mL}$ 2-necked flask was charged with 13 (3.11 g, $14.5 \mathrm{mmol})$ and $\mathrm{CH}_{2} \mathrm{Cl}_{2}$ 
$(154.6 \mathrm{~mL})$ at nitrogen atmosphere, and the solution was cooled to $0{ }^{\circ} \mathrm{C}$. To this solution was added boron tribromide $\left(36.3 \mathrm{~mL}\right.$ of $1.0 \mathrm{M} \mathrm{CH}_{2} \mathrm{Cl}_{2}$ solution, $36.3 \mathrm{mmol}$ ) dropwise at $0{ }^{\circ} \mathrm{C}$, and the mixture was stirred at room temperature for $4 \mathrm{~h}$. After the reaction was quenched with methanol, and the mixture was evaporated, small amount of toluene was added to the residue, and the mixture was again concentrated under reduced pressure. The residue was subjected to column chromatography on silica gel $(n$-hexane/EtOAc $=5 / 1)$ to give $\mathbf{1 4}$ as a white solid (2.85 g, 98\% yield); mp. 96-97 ${ }^{\circ} \mathrm{C} ;{ }^{1} \mathrm{H}$ NMR (500 MHz, $\left.\mathrm{CDCl}_{3}\right) \delta(\mathrm{ppm}) 7.68(\mathrm{~d}, J=9.0 \mathrm{~Hz}$, $1 \mathrm{H}), 7.60(\mathrm{~d}, J=8.5 \mathrm{~Hz}, 1 \mathrm{H}), 7.54(\mathrm{~s}, 1 \mathrm{H}), 7.29(\mathrm{dd}, J=8.5,1.5 \mathrm{~Hz}, 1 \mathrm{H}), 7.11(\mathrm{~d}, J=2.5 \mathrm{~Hz}$, $1 \mathrm{H}), 7.07(\mathrm{dd}, J=9.0,2.5 \mathrm{~Hz}, 1 \mathrm{H}), 4.81(\mathrm{~s}, 1 \mathrm{H}), 2.73(\mathrm{t}, J=7.5 \mathrm{~Hz}, 2 \mathrm{H}), 1.70-1.63(\mathrm{~m}, 2 \mathrm{H})$, 1.39 (sextet, $J=7.5 \mathrm{~Hz}, 2 \mathrm{H}) ; 0.94(\mathrm{t}, J=7.5 \mathrm{~Hz}, 3 \mathrm{H}) ;{ }^{13} \mathrm{C} \mathrm{NMR}\left(126 \mathrm{MHz}, \mathrm{CDCl}_{3}\right) \delta(\mathrm{ppm})$ $152.6,138.1,132.9,129.3,129.1,128.1,126.2$, 126.1 , 117.6, 109.3, 35.6, 33.6, 22.4 14.0; IR (ATR) 3282, 3032, 2958, 2920, 2872, 2848, 1604, 1455, 1363, 1227, $817 \mathrm{~cm}^{-1}$; HRMS (ESI+): $m / z$ Calcd. for $\mathrm{C}_{14} \mathrm{H}_{17} \mathrm{O}[\mathrm{M}+\mathrm{H}]^{+}:$201.12739. Found: 201.12743; Anal. Calcd. for $\mathrm{C}_{14} \mathrm{H}_{16} \mathrm{O}: \mathrm{C}, 83.96 ; \mathrm{H}, 8$.05. Found: C, 83.90; H, 8.22.

\section{Preparation of $15^{8)}$}

A $100 \mathrm{~mL}$ flask was charged with $14(2.84 \mathrm{~g}, 14.2 \mathrm{mmol})$ and methanol $(71.0 \mathrm{~mL})$, and sulfuric acid $(1.1 \mathrm{~mL}, 21.3 \mathrm{mmol})$ was added dropwise to the solution at $0{ }^{\circ} \mathrm{C}$. To this solution were added potassium iodide $(2.36 \mathrm{~g}, 14.2 \mathrm{mmol})$ and $30 \%$ hydrogen peroxide aq. $(1.1 \mathrm{~mL})$ at intact temperature, and the mixture was stirred at room temperature for $3 \mathrm{~h}$. After the reaction was quenched with $0.1 \mathrm{M} \mathrm{NaHSO}_{3}$ aq., and extracted with chloroform. The organic layer was washed with water and brine, and dried over $\mathrm{Na}_{2} \mathrm{SO}_{4}$. After evaporation, the residue was subjected to column chromatography on silica gel (chloroform) to give $\mathbf{1 5}$ as a pale brown solid (4.30 g, 93\% yield); mp. 51-53 ${ }^{\circ} \mathrm{C} ;{ }^{1} \mathrm{H}$ NMR (500 MHz, $\left.\mathrm{CDCl}_{3}\right) \delta(\mathrm{ppm}) 7.82(\mathrm{~d}, J=$ $8.5 \mathrm{~Hz}, 1 \mathrm{H}), 7.65(\mathrm{~d}, J=8.5 \mathrm{~Hz}, 1 \mathrm{H}), 7.51(\mathrm{~s}, 1 \mathrm{H}), 7.39(\mathrm{dd}, J=8.5,1.5 \mathrm{~Hz}, 1 \mathrm{H}), 7.21(\mathrm{~d}, J=$ $8.5 \mathrm{~Hz}, 1 \mathrm{H}), 5.68(\mathrm{~s}, 1 \mathrm{H}), 2.76(\mathrm{t}, J=7.5 \mathrm{~Hz}, 2 \mathrm{H}), 1.71-1.64(\mathrm{~m}, 2 \mathrm{H}), 1.38$ (sextet, $J=7.5 \mathrm{~Hz}$, $2 \mathrm{H}), 0.94(\mathrm{t}, J=7.5 \mathrm{~Hz}, 3 \mathrm{H}) ;{ }^{13} \mathrm{C} \mathrm{NMR}\left(126 \mathrm{MHz}, \mathrm{CDCl}_{3}\right) \delta$ (ppm) 153.3, 139.0, 133.4, 130.35, 130.31, 130.0, 129.9, 127.0, 116.6, 86.3, 35.4, 33.7, 22.6, 14.2; IR (ATR) 3413, 3053, 3026, 2954, 2928, 2855, 1602, 1465, 1344, 1283, 1231, 799, $665 \mathrm{~cm}^{-1}$; HRMS (ESI+): $\mathrm{m} / \mathrm{z}$ Calcd. for $\mathrm{C}_{14} \mathrm{H}_{15} \mathrm{IO}[\mathrm{M}]^{+}: 326.01621$. Found: 326.01663 .

\section{Preparation of 16}

An oven-dried $100 \mathrm{~mL}$ 2-necked flask was charged with sodium hydride $60 \%$ in mineral oil, $1.04 \mathrm{~g}, 26.0 \mathrm{mmol})$ and THF $(240 \mathrm{~mL})$ at nitrogen atmosphere, and the suspension was cooled to $0{ }^{\circ} \mathrm{C}$. To this suspension was added $15(4.24 \mathrm{~g}, 13.0 \mathrm{mmol})$ in THF $(130 \mathrm{~mL})$ and chloromethyl methyl ether $(2.47 \mathrm{~mL}, 32.5 \mathrm{mmol})$ dropwise at $0{ }^{\circ} \mathrm{C}$, and the mixture was stirred at room temperature for $30 \mathrm{~min}$. After allowed to room temperature, the reaction was quenched with sat. $\mathrm{NH}_{4} \mathrm{Cl}$ aq., and extracted with EtOAc. The organic layer was washed with 
sat. $\mathrm{NH}_{4} \mathrm{Cl}$ aq., sat. $\mathrm{NaHCO}_{3}$ aq., brine, and dried over $\mathrm{Na}_{2} \mathrm{SO}_{4}$. After evaporation, the residue was subjected to column chromatography on silica gel $(n$-hexane/EtOAc $=20 / 1)$ to give $\mathbf{1 6}$ as a pale orange oil (4.26 g, 89\% yield); ${ }^{1} \mathrm{H}$ NMR $\left(500 \mathrm{MHz}, \mathrm{CDCl}_{3}\right) \delta$ (ppm) 8.06 (d, $J=8.5$ $\mathrm{Hz}, 1 \mathrm{H}), 7.71(\mathrm{~d}, J=9.0 \mathrm{~Hz}, 1 \mathrm{H}), 7.51(\mathrm{~s}, 1 \mathrm{H}), 7.39(\mathrm{dd}, J=8.5,1.5 \mathrm{~Hz}, 1 \mathrm{H}), 7.32$ (d, $J=9.0$ $\mathrm{Hz}, 1 \mathrm{H}), 5.34(\mathrm{~s}, 2 \mathrm{H}), 3.57(\mathrm{~s}, 3 \mathrm{H}), 2.76(\mathrm{t}, J=7.5 \mathrm{~Hz}, 2 \mathrm{H}), 1.71-1.64$ (m, 2H), 1.38 (sextet, $J$ $=7.5 \mathrm{~Hz}, 2 \mathrm{H}), 0.94(\mathrm{t}, J=7.5 \mathrm{~Hz}, 3 \mathrm{H}) ;{ }^{13} \mathrm{C} \mathrm{NMR}\left(126 \mathrm{MHz}, \mathrm{CDCl}_{3}\right) \delta$ (ppm) 154.1, 139.4, 134.0, 131.3 , 130.6, 129.62, 129.60, 126.5, 116.1, 95.6, 89.5, 56.6, 35.2, 33.4, 22.3 , 14.0; IR (ATR) 3035, 2955, 2927, 2856, 1597, 1495, 1475, 1239, 1150, 1015, 816, $514 \mathrm{~cm}^{-1}$; HRMS (ESI+): $m / z$ Calcd. for $\mathrm{C}_{16} \mathrm{H}_{19} \mathrm{INaO}_{2}[\mathrm{M}+\mathrm{Na}]^{+}: 393.03219$. Found: 393.03242 .

\section{Preparation of $17 b^{9)}$}

A $50 \mathrm{~mL}$ 2-necked round-bottomed flask equipped with a condenser was charged with $\mathrm{NaH}$ (0.295 mg, $7.37 \mathrm{mmol}), \mathrm{CuI}(80.9 \mathrm{mg}, 0.42 \mathrm{mmol})$, and THF (17 mL) at nitrogen atmosphere. To this suspension was added $16(4.21 \mathrm{~mL}$ of $1.0 \mathrm{M}$ THF solution, $4.21 \mathrm{mmol})$ dropwise at room temperature. After addition, 4,4,5,5-tetramethyl-1,3,2-dioxaborolane $(6.50 \mathrm{~mL}$ of $1.0 \mathrm{M}$ THF solution, $6.50 \mathrm{mmol}$ ) was added at room temperature, and the mixture was refluxed for $14 \mathrm{~h}$. After allowed to room temperature, the mixture was poured onto sat. $\mathrm{NH}_{4} \mathrm{Cl}$ aq. $(50 \mathrm{~mL})$, and extracted with EtOAc (ca. $50 \mathrm{~mL}$ ). The organic layer was washed with $\mathrm{H}_{2} \mathrm{O}$ and brine, and dried over $\mathrm{Na}_{2} \mathrm{SO}_{4}$. After evaporation, the residue was subjected to column chromatography on silica gel $(n$-hexane/EtOAc $=20 / 1)$ to give $\mathbf{1 7 b}$ as a pale yellow oil $(1.29$ g, 84\% yield); ${ }^{1} \mathrm{H}$ NMR (500 MHz, $\left.\mathrm{CDCl}_{3}\right) \delta(\mathrm{ppm}) 7.88(\mathrm{~d}, J=8.5 \mathrm{~Hz}, 1 \mathrm{H}), 7.74(\mathrm{~d}, J=9.0$ $\mathrm{Hz}, 1 \mathrm{H}), 7.52(\mathrm{~s}, 1 \mathrm{H}), 7.30(\mathrm{dd}, J=8.5,2.0 \mathrm{~Hz}, 1 \mathrm{H}), 7.28(\mathrm{~d}, J=9.0 \mathrm{~Hz}, 1 \mathrm{H}), 5.23(\mathrm{~s}, 2 \mathrm{H})$, $3.52(\mathrm{~s}, 3 \mathrm{H}), 2.72(\mathrm{t}, J=7.5 \mathrm{~Hz}, 2 \mathrm{H}), 1.68-1.62(\mathrm{~m}, 2 \mathrm{H}), 1.46(\mathrm{~s}, 12 \mathrm{H}), 1.36$ (sextet, $J=7.5$ $\mathrm{Hz}, 2 \mathrm{H}), 0.93(\mathrm{t}, J=7.5 \mathrm{~Hz}) ;{ }^{13} \mathrm{C} \mathrm{NMR}\left(126 \mathrm{MHz}, \mathrm{CDCl}_{3}\right) \delta(\mathrm{ppm}) 158.6,138.3,135.4,130.9$, $129.8,128.2,126.9,126.6,116.3,95.6,83.9,56.2,35.5,33.5,25.0,22.3,14.0$; IR (ATR) 3069, 2977, 2955, 2930, 2856, 1591, 1504, 1305, 1235, 1137, 1030, 854, 756, $667 \mathrm{~cm}^{-1}$; HRMS (ESI+): $m / z$ Calcd. for $\mathrm{C}_{22} \mathrm{H}_{31} \mathrm{BNaO}_{4}[\mathrm{M}+\mathrm{Na}]^{+}:$393.22076. Found: 393.22102.

\section{Preparation of 18}

A $100 \mathrm{~mL}$ 2-necked round-bottomed flask equipped with a condenser was charged with p-diiodobenzene (306.8 mg, $0.93 \mathrm{mmol}$ ), $\mathrm{PdCl}_{2}(\mathrm{dppf}) \cdot \mathrm{CH}_{2} \mathrm{Cl}_{2}$ (76.3 $\left.\mathrm{mg}, 0.093 \mathrm{mmol}\right), \mathbf{1 7 b}$ $(1.03 \mathrm{~g}, 2.79 \mathrm{mmol})$, and THF (34 mL) at nitrogen atmosphere. To this solution was added 2.0 $\mathrm{M} \mathrm{Na}_{2} \mathrm{CO}_{3}$ aq. ( $7 \mathrm{~mL}$ ), and the mixture was refluxed for $20 \mathrm{~h}$. After allowed to room temperature, the mixture was poured onto sat. $\mathrm{NH}_{4} \mathrm{Cl}$ aq. $(50 \mathrm{~mL})$, and extracted with EtOAc (ca. $50 \mathrm{~mL}$ ). The organic layer was washed with sat. $\mathrm{NaHCO}_{3}$ aq. and brine, and dried over $\mathrm{Na}_{2} \mathrm{SO}_{4}$. After evaporation, the residue was passed through a $\mathrm{SH}$ silica gel (purchased from Fuji Silysia Chemical Ltd.). After evaporation, the residue was subjected to column chromatography on silica gel $(n$-hexane/EtOAc $=20 / 1)$ to give $\mathbf{1 8}$ as a white solid $(422 \mathrm{mg}$, 
$81 \%$ yield, as a ca. $1: 1$ mixture of rotamers); mp. $119-120{ }^{\circ} \mathrm{C} ;{ }^{1} \mathrm{H} \mathrm{NMR}\left(500 \mathrm{MHz}, \mathrm{CDCl}_{3}\right) \delta$ (ppm) $7.81(\mathrm{~d}, J=9.0 \mathrm{~Hz}, 2 \mathrm{H}), 7.67(\mathrm{~d}, J=8.5 \mathrm{~Hz}, 1 \mathrm{H}), 7.63(\mathrm{~s}, 2 \mathrm{H}), 7.60(\mathrm{~d}, J=8.5 \mathrm{~Hz}, 1 \mathrm{H})$, $7.52(\mathrm{~s}, 2 \mathrm{H}), 7.51(\mathrm{~s}, 2 \mathrm{H}), 7.47(\mathrm{~d}, J=9.0 \mathrm{~Hz}, 1 \mathrm{H}), 7.45(\mathrm{~d}, J=9.0 \mathrm{~Hz}, 1 \mathrm{H}), 7.31(\mathrm{dd}, J=9.0$, $1.5 \mathrm{~Hz}, 1 \mathrm{H}), 7.25$ (dd, $J=9.0,1.5 \mathrm{~Hz}, 1 \mathrm{H}), 5.16$ (s, 2H), 5.09 (s, 2H), 3.39 (s, 3H), 3.31 (s, $3 \mathrm{H}), 2.77(\mathrm{t}, J=8.0 \mathrm{~Hz}$ ) and $2.76(\mathrm{t}, J=8.0 \mathrm{~Hz}$, total $4 \mathrm{H}$ ), 1.69 (quint, $J=7.5 \mathrm{~Hz}$ ) and 1.68 (quint, $J=7.5 \mathrm{~Hz}$, total $4 \mathrm{H}), 1.43-1.37(\mathrm{~m}, 4 \mathrm{H}), 0.952(\mathrm{t}, J=7.0 \mathrm{~Hz})$ and $0.946(\mathrm{t}, J=7.0 \mathrm{~Hz}$, total $6 \mathrm{H}) ;{ }^{13} \mathrm{C} \mathrm{NMR}\left(126 \mathrm{MHz}, \mathrm{CDCl}_{3}\right) \delta(\mathrm{ppm}) 150.9,138.73,138.70,135.11,135.08$, $132.01,131.98,130.70,130.68,130.3$, 128.5 , 128.0 , 127.9, 127.7 , 127.6, 126.3 , 125.6, 125.5, 118.0, 117.9, 96.2, 96.1, 56.2, 56.1, 35.5, 33.5, 22.4, 14.0; IR (ATR) 3066, 3031, 2955, 2928, 2853, 1593, 1478, 1376, 1336, 1231, 1147, 1077, 1000, 903, $824 \mathrm{~cm}^{-1}$; HRMS (ESI+): $\mathrm{m} / \mathrm{z}$ Calcd. for $\mathrm{C}_{38} \mathrm{H}_{42} \mathrm{NaO}_{4}[\mathrm{M}+\mathrm{Na}]^{+}:$585.29753. Found: 585.29761; Anal. Calcd. for $\mathrm{C}_{38} \mathrm{H}_{42} \mathrm{O}_{4}: \mathrm{C}$, 81.10; H, 7.52. Found: C, 80.92; H, 7.61.

\section{Preparation of $1 b^{10)}$}

A branched test tube was charged with $18(225.1 \mathrm{mg}, 0.40 \mathrm{mmol})$ and THF $(2 \mathrm{~mL})$ at nitrogen atmosphere, and the solution was cooled to $-78{ }^{\circ} \mathrm{C}$. To this solution was added $n$-BuLi $(0.58 \mathrm{~mL}$ of $1.65 \mathrm{M} n$-hexane solution, $0.96 \mathrm{mmol})$ dropwise at $-78{ }^{\circ} \mathrm{C}$, and the mixture was stirred at $0{ }^{\circ} \mathrm{C}$ for $2 \mathrm{~h}$. After cooled to $-78^{\circ} \mathrm{C}$, trimethoxyborane $(140 \mu \mathrm{L}, 1.2$ mmol) was added dropwise at $-78{ }^{\circ} \mathrm{C}$, and mixture was stirred at room temperature for $24 \mathrm{~h}$. After the volatiles were removed under reduced pressure, to the residue was added benzene ( 2 $\mathrm{mL})$ and $30 \% \mathrm{H}_{2} \mathrm{O}_{2}$ aq. (120 $\left.\mu \mathrm{L}\right)$, and the mixture was refluxed for $5 \mathrm{~h}$. After allowed to room temperature, the reaction was quenched by $10 \% \mathrm{NaHSO}_{3}$ aq., and the organic layer was separated from aqueous layer. The organic layer was washed with water and brine, and dried with $\mathrm{Na}_{2} \mathrm{SO}_{4}$. After evaporation, the reside was subjected to column chromatography on silica gel $(n$-hexane/EtOAc $=5 / 1)$ to give $\mathbf{1 b}$ as a pale orange solid $(215.7 \mathrm{mg}, 89 \%$ yield , as a ca. 1:1 mixture of rotamers); mp. $140-142{ }^{\circ} \mathrm{C} ;{ }^{1} \mathrm{H}$ NMR $\left(500 \mathrm{MHz}, \mathrm{CDCl}_{3}\right) \delta(\mathrm{ppm}) 7.54-7.43$ $(\mathrm{m}, 8 \mathrm{H}), 7.36(\mathrm{~s}, 2 \mathrm{H}), 7.17(\mathrm{~d}, J=9.5 \mathrm{~Hz})$ and $7.13(\mathrm{~d}, J=8.5 \mathrm{~Hz}$, total $2 \mathrm{H}), 7.03(\mathrm{~s})$ and 7.01 $(\mathrm{s}$, total $2 \mathrm{H}), 4.86(\mathrm{~s})$ and $4.80(\mathrm{~s}$, total $4 \mathrm{H}), 3.50(\mathrm{~s})$ and $3.48(\mathrm{~s}$, total $6 \mathrm{H}), 2.80-2.71(\mathrm{~m}, 4 \mathrm{H})$, $1.72-1.64(\mathrm{~m}, 4 \mathrm{H}), 1.42-1.35(\mathrm{~m}, 4 \mathrm{H}), 0.95(\mathrm{t}, J=7.5 \mathrm{~Hz}, 6 \mathrm{H}) ;{ }^{13} \mathrm{C} \mathrm{NMR}\left(126 \mathrm{MHz}, \mathrm{CDCl}_{3}\right)$ $\delta$ (ppm) 147.9, 147.8, 143.03, 142.99, 140.4, 140.3, 135.14, 135.07, 132.4, 131.0, 130.7, 130.6, 126.4 , 126.3 , 125.7, 125.6, 125.5, 125.3, 125.2, 110.6, 100.2, 100.1, 57.62, 57.57, 35.6, 33.5, 22.4, 14.0; IR (ATR) 3382, 3065, 2957, 2929, 2856, 1629, 1502, 1463, 1430, 1395, 1240, 1155, 967, 899, $819 \mathrm{~cm}^{-1}$; HRMS (ESI+): $\mathrm{m} / z$ Calcd. for $\mathrm{C}_{38} \mathrm{H}_{42} \mathrm{O}_{6} \mathrm{Na}[\mathrm{M}+\mathrm{Na}]^{+}$: 617.28736. Found: 617.28737; Anal. Calcd. for $\mathrm{C}_{38} \mathrm{H}_{42} \mathrm{O}_{6} \cdot 0.5 \mathrm{H}_{2} \mathrm{O}: \mathrm{C}, 75.60 ; \mathrm{H}, 7.18$. Found: C, 75.50; H, 7.04. 


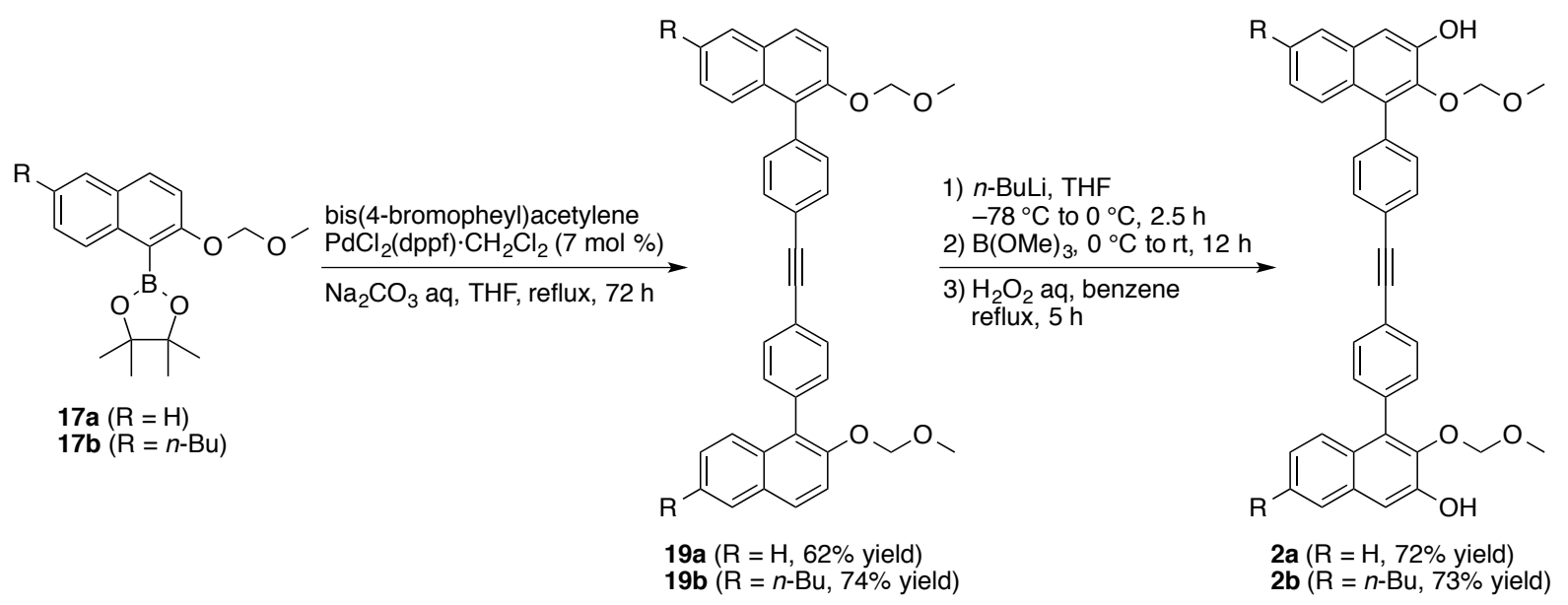

\section{Preparation of 19a: a General Procedure}

A $200 \mathrm{~mL}$ 2-necked round-bottomed flask was charged with bis(4-bromophenyl)acetylene (0.94 g, $2.8 \mathrm{mmol}), \mathrm{PdCl}_{2}(\mathrm{dppf}) \cdot \mathrm{CH}_{2} \mathrm{Cl}_{2}(190 \mathrm{mg}, 0.20 \mathrm{mmol}), \mathbf{1 7 a}(2.20 \mathrm{~g}, 7.0 \mathrm{mmol})$, and THF (90 mL) under nitrogen atmosphere. To this solution was added $2.0 \mathrm{M} \mathrm{Na}_{2} \mathrm{CO}_{3}$ aq. (17.5 $\mathrm{mL}$ ), and the mixture was refluxed for $72 \mathrm{~h}$. After allowed to room temperature, the mixture was poured onto $\mathrm{H}_{2} \mathrm{O}(200 \mathrm{~mL})$, and extracted with EtOAc (ca. $\left.300 \mathrm{~mL}\right)$. The organic layer was washed with brine, and dried over $\mathrm{Na}_{2} \mathrm{SO}_{4}$. After evaporation, the residue was subjected to column chromatography on silica gel $(n$-hexane/EtOAc $=5 / 1)$ and recrystallized from $\mathrm{CHCl}_{3} / n$-hexene to give 19a as a white solid $(8.34 \mathrm{~g}, 62 \%$ yield $)$; mp. $188-189^{\circ} \mathrm{C} ;{ }^{1} \mathrm{H} \mathrm{NMR}$ $\left(500 \mathrm{MHz}, \mathrm{CDCl}_{3}\right) \delta(\mathrm{ppm}) 7.87(\mathrm{~d}, J=9.0 \mathrm{~Hz}, 2 \mathrm{H}), 7.86-7.84(\mathrm{~m}, 2 \mathrm{H}), 7.70(\mathrm{~d}, J=8.5 \mathrm{~Hz}$, 4H), 7.55-7.33 (m, 2H), $7.49(\mathrm{~d}, J=9.0 \mathrm{~Hz}, 2 \mathrm{H}), 7.41(\mathrm{~d}, J=8.5 \mathrm{~Hz}, 4 \mathrm{H}), 7.39-7.37(\mathrm{~m}, 4 \mathrm{H})$, $5.13(\mathrm{~s}, 4 \mathrm{H}), 3.34(\mathrm{~s}, 6 \mathrm{H}) ;{ }^{13} \mathrm{C} \mathrm{NMR}\left(126 \mathrm{MHz}, \mathrm{CDCl}_{3}\right) \delta(\mathrm{ppm}) 151.5,136.8,133.5,131.6$, $131.3,130.0,129.5,128.1,126.7,126.6,125.5,124.3,122.2,117.3,95.7,89.8,56.3$; IR (ATR) 3199, 1952, 1083, 1000, 834, $811 \mathrm{~cm}^{-1}$; HRMS (ESI+): $\mathrm{m} / z$ Calcd. for $\mathrm{C}_{38} \mathrm{H}_{30} \mathrm{O}_{4}$ $(\mathrm{M}+\mathrm{Na})^{+}:$573.2036. Found 573.2080; Anal. Calcd. for $\mathrm{C}_{38} \mathrm{H}_{30} \mathrm{O}_{4}: \mathrm{C}, 82.89 ; \mathrm{H}, 5.49$. Found: $\mathrm{C}$, 82.37; H, 5.30 .

19b: $74 \%$ yield as a white solid; mp. $133-134{ }^{\circ} \mathrm{C} ;{ }^{1} \mathrm{H}$ NMR $\left(500 \mathrm{MHz}, \mathrm{CDCl}_{3}\right) \delta(\mathrm{ppm}) 7.79$ $(\mathrm{d}, J=9.0 \mathrm{~Hz}, 2 \mathrm{H}), 7.69(\mathrm{~d}, J=9.0 \mathrm{~Hz}, 4 \mathrm{H}), 7.61(\mathrm{~s}, 2 \mathrm{H}), 7.46(\mathrm{~d}, J=8.5 \mathrm{~Hz}, 2 \mathrm{H}), 7.44(\mathrm{~d}, J$ $=9.0 \mathrm{~Hz}, 2 \mathrm{H}), 7.40(\mathrm{~d}, J=8.5 \mathrm{~Hz}, 4 \mathrm{H}), 7.23(\mathrm{~d}, J=8.5 \mathrm{~Hz}, 2 \mathrm{H}), 5.10(\mathrm{~s}, 4 \mathrm{H}), 3.32(\mathrm{~s}, 6 \mathrm{H})$, $2.75(\mathrm{t}, J=5.0 \mathrm{~Hz}, 4 \mathrm{H}), 1.70-1.64(\mathrm{~m}, 4 \mathrm{H}), 1.40$ (sextet, $J=7.5 \mathrm{~Hz}, 2 \mathrm{H}), 0.94(\mathrm{t}, J=7.3 \mathrm{~Hz}$, $6 \mathrm{H}) ;{ }^{13} \mathrm{C}$ NMR $\left(126 \mathrm{MHz}, \mathrm{DMSO}-d_{6}\right) \delta(\mathrm{ppm}) 150.9,138.9,136.9,131.8,131.5,131.2,130.2$, 128.9.3, 128.2, 126.7, 126.5, 125.4, 122.1, 117.4, 95.8, 89.8, 56.2.4, 35.6; IR (ATR) 2953, 2022, 1594, 1493, 1070, 1027, 836 $\mathrm{cm}^{-1}$; HRMS (ESI+): $m / z$. Calcd. for $\mathrm{C}_{46} \mathrm{H}_{46} \mathrm{Na}_{1} \mathrm{O}_{4}[\mathrm{M}+\mathrm{Na}]^{+}$: 685.32938. Found: 685.33017; Anal. Calcd. for $\mathrm{C}_{46} \mathrm{H}_{46} \mathrm{O}_{4}$ : C, 83.35; H, 7.00, Found: C, 83.56; H, 7.10.

\section{Preparation of 2a: a General Procedure}


A $50 \mathrm{~mL}$ 2-necked round-bottomed flask was charged with 19a $(0.72 \mathrm{~g}, 1.31 \mathrm{mmol})$, and THF $(7 \mathrm{~mL})$ at nitrogen atmosphere, and the solution was cooled to $-78{ }^{\circ} \mathrm{C}$. To this solution was added $n$-BuLi $(2.5 \mathrm{~mL}$ of $1.63 \mathrm{M} n$-hexane solution, $0.4 \mathrm{mmol})$ dropwise at $-78{ }^{\circ} \mathrm{C}$, and the mixture was stirred at $0{ }^{\circ} \mathrm{C}$ for $2.5 \mathrm{~h}$. Trimethoxyborane $(0.4 \mathrm{~mL}, 3.93 \mathrm{mmol})$ was then added dropwise at intact temperature, and the mixture was stirred at room temperature for 12 h. After the volatiles were removed under reduced pressure, benzene $(6.8 \mathrm{~mL})$ and $30 \% \mathrm{H}_{2} \mathrm{O}_{2}$ aq. $(0.4 \mathrm{~mL})$ was added to the residue, and the mixture was refluxed for $5 \mathrm{~h}$. After allowed to room temperature, the mixture was filtered, and the precipitate was washed with $\mathrm{CHCl}_{3}$ and $\mathrm{H}_{2} \mathrm{O}$ to give $2 \mathrm{a}$ as a pale pink solid $\left(0.55 \mathrm{~g}\right.$, $72 \%$ yield); mp. $205-207{ }^{\circ} \mathrm{C}$ (decomp.); ${ }^{1} \mathrm{H}$ NMR $\left(500 \mathrm{MHz}, \mathrm{CDCl}_{3}\right) \delta(\mathrm{ppm}) 7.74(\mathrm{~d}, J=8.5 \mathrm{~Hz}, 2 \mathrm{H}), 7.72(\mathrm{~d}, J=8.5 \mathrm{~Hz}, 4 \mathrm{H}), 7.47(\mathrm{~d}, J=8.5$ $\mathrm{Hz}, 2 \mathrm{H}), 7.42-7.38(\mathrm{~m}, 6 \mathrm{H}), 7.26-7.15(\mathrm{~m}, 2 \mathrm{H}), 7.10(\mathrm{~s}, 2 \mathrm{H}), 4.78(\mathrm{~s}, 4 \mathrm{H}), 3.46(\mathrm{~s}, 6 \mathrm{H}) ;{ }^{13} \mathrm{C}$ NMR $\left(126 \mathrm{MHz}, \mathrm{CDCl}_{3}\right) \delta(\mathrm{ppm}) 147.9,143.7 .0,136.1,132.2,131.7,130.9,130.9,127.8$, 126.7, 125.7, 125.6, 124.0, 122.5, 111.3, 100.1, 89.8, 57.6; IR (ATR) 3352, 2969, 1739, 1447 , 1366, 1216, 1096, $771 \mathrm{~cm}^{-1}$; HRMS (ESI+): $\mathrm{m} / z$ Calcd. for $\mathrm{C}_{38} \mathrm{H}_{30} \mathrm{O}_{4}(\mathrm{M}+\mathrm{Na})^{+}: 605.1953$. Found 605.1935.

2b: A $50 \mathrm{~mL}$ 2-necked round-bottomed flask was charged with $\mathbf{1 9 b}(0.91 \mathrm{~g}, 1.38 \mathrm{mmol})$, and THF $(7 \mathrm{~mL})$ at nitrogen atmosphere, and the solution was cooled to $-78{ }^{\circ} \mathrm{C}$. To this solution was added $n$-BuLi (2.7 $\mathrm{mL}$ of $1.60 \mathrm{M} n$-hexane solution, $0.43 \mathrm{mmol})$ dropwise at $-78^{\circ} \mathrm{C}$, and the mixture was stirred at $0{ }^{\circ} \mathrm{C}$ for $2.5 \mathrm{~h}$. Trimethoxyborane $(0.5 \mathrm{~mL}, 4.14 \mathrm{mmol})$ was then added dropwise at intact temperature, and the mixture was stirred at room temperature for 12 h. After the volatiles were removed under reduced pressure, benzene $(7.2 \mathrm{~mL})$ and $30 \% \mathrm{H}_{2} \mathrm{O}_{2}$ aq. $(0.5 \mathrm{~mL})$ was added to the residue, and the mixture was refluxed for $5 \mathrm{~h}$. After allowed to room temperature, the mixture was poured onto $\mathrm{H}_{2} \mathrm{O}(50 \mathrm{~mL})$, and extracted with EtOAc (ca. $50 \mathrm{~mL}$ ). The organic layer was washed with brine, and dried over $\mathrm{Na}_{2} \mathrm{SO}_{4}$. After evaporation, the residue was subjected to column chromatography on silica gel $(n$-hexane/EtOAc $=3 / 1)$ to give $2 \mathbf{b}$ as a pink solid $(0.70 \mathrm{~g}, 73 \%$ yield $)$; mp. $110-112{ }^{\circ} \mathrm{C} ;{ }^{1} \mathrm{H}$ NMR $\left(500 \mathrm{MHz}, \mathrm{CDCl}_{3}\right) \delta$ (ppm) $7.71(\mathrm{~d}, J=6.25 \mathrm{~Hz}, 4 \mathrm{H}), 7.51(2,2 \mathrm{H}), 7.40(\mathrm{~d}, J=6.5 \mathrm{~Hz}, 4 \mathrm{H}), 7.39$ (d, $J=9.0 \mathrm{~Hz}$, 2H), 7.34 (s, 2H), $7.10(\mathrm{~d}, J=8.5 \mathrm{~Hz}, 2 \mathrm{H}), 7.05(\mathrm{~s}, 2 \mathrm{H}), 4.76(\mathrm{~s}, 4 \mathrm{H}), 3.45(\mathrm{~s}, 6 \mathrm{H}), 2.72(\mathrm{t}, J=$ $7.8 \mathrm{~Hz}, 4 \mathrm{H}), 1.66(\mathrm{~m}, 4 \mathrm{H}), 1.38$ (sextet, $J=7.5 \mathrm{~Hz}, 4 \mathrm{H}), 0.94(\mathrm{t}, J=7.3 \mathrm{~Hz}, 6 \mathrm{H}) ;{ }^{13} \mathrm{C} \mathrm{NMR}$ $\left(126 \mathrm{MHz}, \mathrm{CDCl}_{3}\right) \delta(\mathrm{ppm}) 147.9,143.1,140.5,136.3,132.5,131.7,131.0,130.8,126.2$, $125.7,125.6,125.6,125.3,122.5,110.9,100.3,89.9,57.7,35.7,33.6,22.5,14,1$; IR (ATR) 3361, 2934, 1744, 1367, 1216, 1070, 829, $779 \mathrm{~cm}^{-1}$; HRMS (ESI+): $\mathrm{m} / \mathrm{z}$ Calcd. for $\mathrm{C}_{46} \mathrm{H}_{46} \mathrm{~K}_{1} \mathrm{O}_{6}[\mathrm{M}+\mathrm{K}]^{+}:$733.29314. Found: 733.29371; Anal. Calcd. for $\mathrm{C}_{46} \mathrm{H}_{46} \mathrm{O}_{6}: \mathrm{C}, 79.51 ; \mathrm{H}$, 6.67. Found: C, 79.66; H, 6.60. 


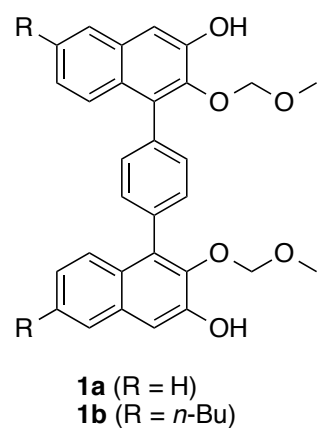

or

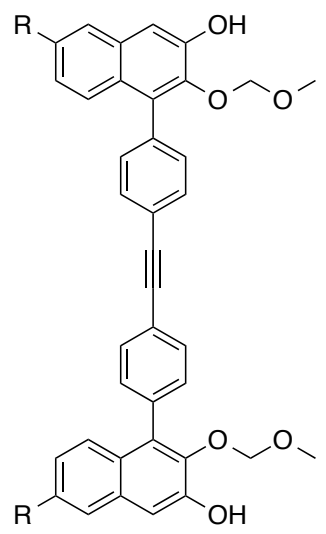

2a $(\mathrm{R}=\mathrm{H})$ $2 \mathrm{~b}(\mathrm{R}=n-\mathrm{Bu})$

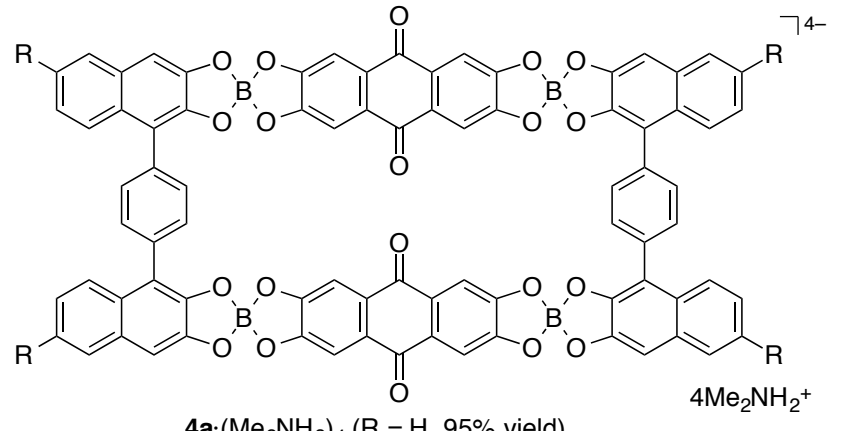

$4 a \cdot\left(\mathrm{Me}_{2} \mathrm{NH}_{2}\right)(\mathrm{R}=\mathrm{H}, 95 \%$ yield $)$

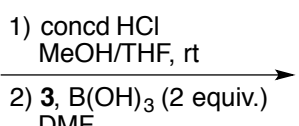

4b $\left(\mathrm{Me}_{2} \mathrm{NH}_{2}\right)_{4}(\mathrm{R}=n-\mathrm{Bu}, 93 \%$ yield $)$

or

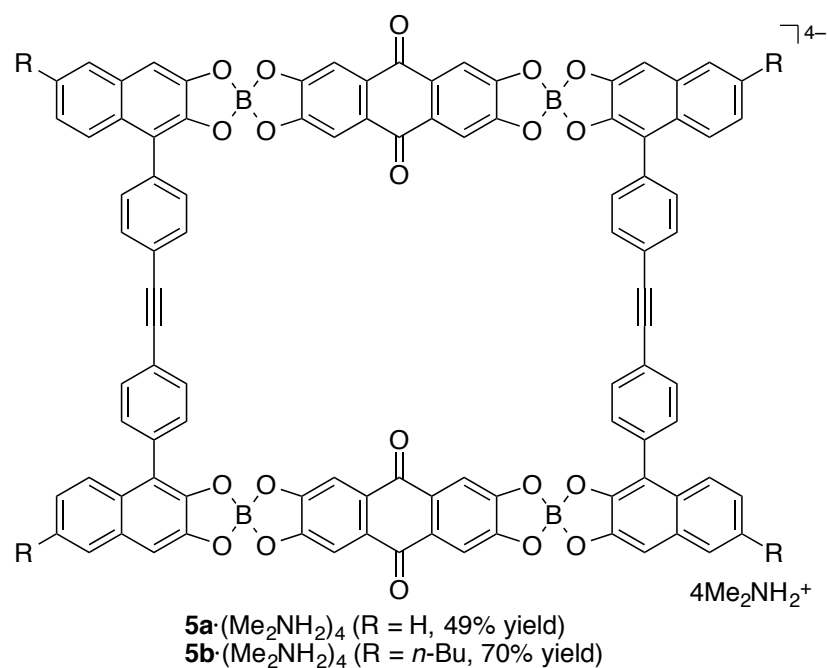

$\mathbf{5 b} \cdot\left(\mathrm{Me}_{2} \mathrm{NH}_{2}\right)_{4}(\mathrm{R}=n-\mathrm{Bu}, 70 \%$ yield $)$

\section{Preparation of $4 \mathrm{a} \cdot\left(\mathrm{Me}_{2} \mathrm{NH}_{2}\right)_{4}$ : a General Procedure ${ }^{11)}$}

In a $10 \mathrm{~mL}$ branched test tube, $1 \mathbf{a}(48.3 \mathrm{mg}, 0.1 \mathrm{mmol})$ was suspended in MeOH/THF (1:1 $(\mathrm{v} / \mathrm{v}), 3 \mathrm{~mL})$, and conc. $\mathrm{HCl}$ aq. $(0.26 \mathrm{~mL})$ was added dropwise at $0{ }^{\circ} \mathrm{C}$. After stirred at room temperature for $3 \mathrm{~h}$, the volatiles were removed under reduced pressure. The residual pale pink powder (1a'), boric acid (12.4 $\mathrm{mg}, 0.2 \mathrm{mmol})$, and $\mathbf{3}(27.2 \mathrm{mg}, 0.1 \mathrm{mmol})$ were then dissolved in DMF $(2.5 \mathrm{~mL})$ at nitrogen atmosphere, and the mixture was stirred at $150{ }^{\circ} \mathrm{C}$ for $14 \mathrm{~h}$. After allowed to room temperature, the resulting solution was concentrated under the pressure, and the residue was washed by $\mathrm{Et}_{2} \mathrm{O}$. The precipitate was collected by filtration, and under reduced pressure to give as a dark brown solid $(73.2 \mathrm{mg}, 95 \%$ yield $)$; mp. $>240{ }^{\circ} \mathrm{C}$ (decomp.); ${ }^{1} \mathrm{H}$ NMR (500 MHz, DMSO- $d_{6}$ ) $\delta 8.13$ (br s, 8H), 7.71-7.69 (m, 4H), 7.68-7.66 $(\mathrm{m}, 4 \mathrm{H}), 7.53(\mathrm{~s}, 8 \mathrm{H}), 7.22-7.20(\mathrm{~m}, 8 \mathrm{H}), 7.07(\mathrm{~s}, 8 \mathrm{H}), 6.96(\mathrm{~s}, 4 \mathrm{H}) ;{ }^{13} \mathrm{C}$ NMR $(126 \mathrm{MHz}$, DMSO $\left.-d_{6}\right) \delta 182.4,156.5,152.1,150.1,134.6,131.0,130.1,128.4,127.9,127.0,124.1$, 122.7, 122.6, 115.4, 105.1, 102.6, 34.8; IR (ATR) 3486, 3122, 1655, 1459, 1328, 1242, 1047, 930, $744 \mathrm{~cm}^{-1}$; HRMS (ESI-): $\mathrm{m} / \mathrm{z}$. Calcd. for $\mathrm{C}_{80} \mathrm{H}_{36} \mathrm{~B}_{4} \mathrm{O}_{20}[\mathrm{M}]^{4-}: 340.05431$. Found: 340.05534 .

4b· $\left(\mathbf{M e}_{2} \mathbf{N H}_{2}\right)_{4}: 93 \%$ yield as a dark brown solid (15 h); mp. $>249{ }^{\circ} \mathrm{C}$ (decomp.); ${ }^{1} \mathrm{H}$ NMR $\left(500 \mathrm{MHz}, \mathrm{DMSO}-d_{6}\right) \delta(\mathrm{ppm}) 8.09(\mathrm{br} \mathrm{s}, 8 \mathrm{H}), 7.61(\mathrm{~d}, J=8.5 \mathrm{~Hz}, 4 \mathrm{H}), 7.51(\mathrm{~s}, 8 \mathrm{H}), 7.44(\mathrm{~s}$, $4 \mathrm{H}), 7.07-7.06(\mathrm{~s}, \mathrm{~d}, 12 \mathrm{H}), 6.88(\mathrm{~s}, 4 \mathrm{H}), 2.67$ (t, $J=7.5 \mathrm{~Hz}, 8 \mathrm{H}), 1.64$ (quint, $J=7.5 \mathrm{~Hz}, 8 \mathrm{H}$ ), 
1.37 (sextet, $J=7.5 \mathrm{~Hz}, 8 \mathrm{H}), 0.93(\mathrm{t}, J=7.5 \mathrm{~Hz}, 12 \mathrm{H}) ;{ }^{13} \mathrm{C} \mathrm{NMR}\left(126 \mathrm{MHz}, \mathrm{DMSO}-d_{6}\right) \delta$ (ppm) 182.4, 156.6, 152.1, 149.4, 136.2, 134.6, 130.9, 130.2, 127.8, 126.7, 125.7, 124.1, 123.9, 115.3, 105.1, 102.3, 35.3, 34.9, 33.8, 22.4, 14.4; IR (ATR) 3636, 3095, 1656, 1457, 1326, 1227, 1042, 818, $744 \mathrm{~cm}^{-1}$; HRMS (ESI-): $m / z$ Calcd. for $\mathrm{C}_{96} \mathrm{H}_{68} \mathrm{~B}_{4} \mathrm{O}_{20}[\mathrm{M}]^{4-}: 396.11690$. Found: 396.11808.

\section{Preparation of $5 \mathrm{a} \cdot\left(\mathrm{Me}_{2} \mathrm{NH}_{2}\right)_{4}$ : a General Procedure ${ }^{11)}$}

In a $20 \mathrm{~mL}$ test tube, $2 \mathbf{a}(19.7 \mathrm{mg}, 40 \mu \mathrm{mol})$ was suspended in MeOH/THF (1:1 (v/v), 0.8 $\mathrm{mL})$, and conc. $\mathrm{HCl}$ aq. $(0.2 \mathrm{~mL})$ was added dropwise at room temperature. After stirred at intact temperature for $4 \mathrm{~h}$, the volatiles were removed under reduced pressure. The residual white powder (2a'), boric acid $(5.0 \mathrm{mg}, 80 \mu \mathrm{mol})$, and $\mathbf{3}$ were then dissolved in DMF (4.0 $\mathrm{mL}$ ), and the solution was transferred into a $5 \mathrm{~mL}$ branched test tube equipped with a condenser under nitrogen atmosphere. The mixture was stirred at $80{ }^{\circ} \mathrm{C}$ for $94 \mathrm{~h}$. After allowed to room temperature, the resulting solution was concentrated under the pressure, and the residue was washed by $\mathrm{Et}_{2} \mathrm{O}$. The precipitate was collected by filtration, and under reduced pressure to give as a dark brown solid (16.9 $\mathrm{mg}$, 49\% yield). For further purification, the product was converted into its tetra $\left(n\right.$-butylammonium) salt $\left(\mathbf{5 a} \cdot(\mathrm{TBA})_{4}\right)$ by treatment with 4 equiv of TBAOH $\left(10 \mathrm{w} / \mathrm{w} \%\right.$ in $\left.\mathrm{H}_{2} \mathrm{O}\right)$ and reprecipitated by $\mathrm{H}_{2} \mathrm{O} ; \mathrm{mp} .>260{ }^{\circ} \mathrm{C}$ (decomp.); ${ }^{1} \mathrm{H}$ NMR (500 MHz, DMSO- $\left.d_{6}\right) \delta(\mathrm{ppm})$ 7.69-7.65 (m, 14H), 7.50-7.47 (m, 14H), 7.22-7.17 (m, $4 \mathrm{H}), 7.17(\mathrm{~s}, 8 \mathrm{H}), 7.16-7.12(\mathrm{~m}, 4 \mathrm{H}), 6.99(\mathrm{~s}, 4 \mathrm{H}), 3.10-3.05(\mathrm{~m}, 32 \mathrm{H}), 1.50$ (quint, $J=7.5$ $\mathrm{Hz}, 32 \mathrm{H}), 1.25$ (sextet, $J=7.5 \mathrm{~Hz}, 32 \mathrm{H}), 0.88(\mathrm{t}, J=7.0 \mathrm{~Hz}, 48 \mathrm{H}) ;{ }^{13} \mathrm{C} \mathrm{NMR}(126 \mathrm{MHz}$, $\left.\mathrm{CDCl}_{3}\right) \delta(\mathrm{ppm}) 182.3,156.6,151.9,149.9,136.9,131.7,130.1,128.0,127.9,127.1,123.7$, 122.9, 121.1, 111.7, 105.3, 103.2, 90.0, 58.0, 23.5, 19.6, 13.9; IR (ATR) 3476, 2957, 1573, 1458, 1326, 1247, 1044, $749 \mathrm{~cm}^{-1}$; HRMS (ESI-): $\mathrm{m} / \mathrm{z}$ Calcd. for $\mathrm{C}_{96} \mathrm{H}_{44} \mathrm{~B}_{4} \mathrm{O}_{20}[\mathrm{M}]^{4}$ : 1560.27981. Found: 1560.28207.

$\mathbf{5 b} \cdot\left(\mathbf{M e}_{2} \mathbf{N H}_{2}\right)_{4}: 70 \%$ yield as a pale gray solid (72 h); mp. $>214{ }^{\circ} \mathrm{C}$ (decomp.); ${ }^{1} \mathrm{H}$ NMR (500 $\left.\mathrm{MHz}, \mathrm{DMSO}-d_{6}\right) \delta(\mathrm{ppm}) 8.11(\mathrm{br} \mathrm{s}, 8 \mathrm{H}), 7.67(\mathrm{~d}, J=8.0 \mathrm{~Hz}, 18 \mathrm{H}), 7.48(\mathrm{~d}, J=8.0 \mathrm{~Hz}, 8 \mathrm{H})$, $7.44(\mathrm{~s}, 4 \mathrm{H}), 7.40(\mathrm{~d}, J=8.5 \mathrm{~Hz}, 8 \mathrm{H}), 7.15(\mathrm{~s}, 8 \mathrm{H}), 7.00(\mathrm{~d}, J=9.5 \mathrm{~Hz}, 4 \mathrm{H}), 6.91(\mathrm{~s}, 4 \mathrm{H}), 7.12$ (t, $J=8.0 \mathrm{~Hz}, 6 \mathrm{H}), 6.92(\mathrm{~s}, 6 \mathrm{H}), 2.64(\mathrm{t}, J=9.8 \mathrm{~Hz}, 8 \mathrm{H}), 1.62-1.58(\mathrm{~m}, 4 \mathrm{H}), 1.36-1.32(\mathrm{~m}$, $8 \mathrm{H}), 0.91(\mathrm{t}, J=7.8 \mathrm{~Hz}, 12 \mathrm{H}) ;{ }^{13} \mathrm{C}$ NMR $\left(126 \mathrm{MHz}, \mathrm{DMSO}-d_{6}\right) \delta(\mathrm{ppm}) 183.2,157.6,152.9$, $150.2,138.0, \quad 137.4,132.6,131.1,128.8,127.1,126.8,125.1,121.9,115.5,106.2,103.8$, 90.9, 37.2, 36.2, 32.2, 23.3, 15.3; IR (ATR) 3378, 2969, 1738, 1454, 1217, 1044, $772 \mathrm{~cm}^{-1}$; HRMS (ESI-): $m / z$ Calcd. for $\mathrm{C}_{112} \mathrm{H}_{76} \mathrm{~B}_{4} \mathrm{O}_{20}[\mathrm{M}]^{4-}: 446.13255$. Found: 446.13168 . 


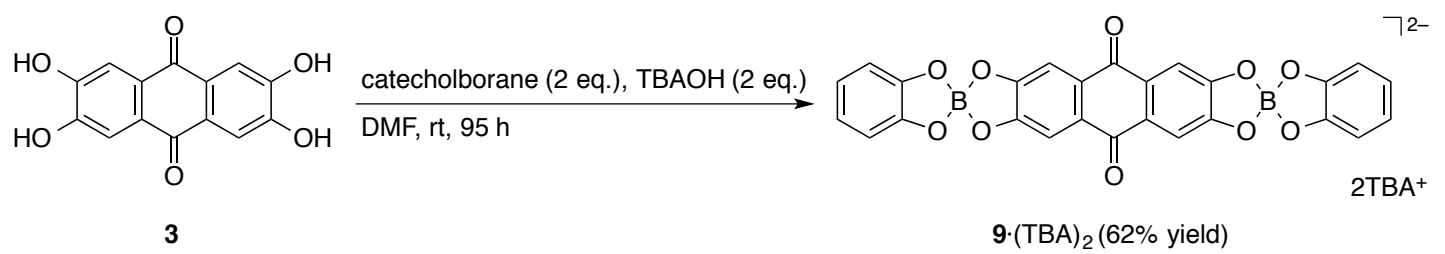

\section{Preparation of 9.(TBA)}

A branched test tube was charged with $3(46.1 \mathrm{mg}, 169 \mu \mathrm{mol})$ and DMF (2.7 mL) at nitrogen atmosphere. To the solution were added tetra(n-butyl)ammonium hydroxide ( $880 \mu \mathrm{L}$ of 10 wt $\%$ aqueous soluton, $339 \mu \mathrm{mol}$ ) and catecholborane (340 $\mu \mathrm{L}$ of 1.0 M THF solution, 339 $\mu \mathrm{mol})$, and the mixture was stirred at room temperature. After $95 \mathrm{~h}$, the solution was poured dropwise into $\mathrm{Et}_{2} \mathrm{O}$ (ca. $50 \mathrm{~mL}$ ) and the resulting precipitate was collected by filtration. The residual solid was washed with $\mathrm{MeOH}$ and dried under reduced pressure to give 9.(TBA) ${ }_{2}$ as a pale yellow solid (104.6 mg, 62\% yield); mp. > $281{ }^{\circ} \mathrm{C}$ (decomp.); ${ }^{1} \mathrm{H}$ NMR (500 MHz, DMSO- $\left.d_{6}\right) \delta(\mathrm{ppm}) 7.14(\mathrm{~s}, 4 \mathrm{H}), 6.55-6.53(\mathrm{~m}, 8 \mathrm{H}), 3.14(\mathrm{t}, J=7.5 \mathrm{~Hz}, 16 \mathrm{H}), 1.55(\mathrm{bs}, 16 \mathrm{H})$, $1.31-1.27(\mathrm{~m}, 16 \mathrm{H}), 0.92(\mathrm{t}, J=7.0 \mathrm{~Hz}, 24 \mathrm{H}) ;{ }^{13} \mathrm{C}$ NMR $\left(126 \mathrm{MHz}, \mathrm{DMSO}-d_{6}\right) \delta(\mathrm{ppm})$ 182.4 156.9, 151.6, 127.8, 118.4, 108.5, 105.0, 58.0, 23.5, 19.7, 14.0; IR (ATR) 3228, 2963, 1486, 1320, 1246, 1225, 1045, 729, $701 \mathrm{~cm}^{-1}$; Anal. Calcd. for $\mathrm{C}_{58} \mathrm{H}_{84} \mathrm{~B}_{2} \mathrm{~N}_{2} \mathrm{O}_{10} \cdot \mathrm{H}_{2} \mathrm{O}: \mathrm{C}, 69.05$; H, 8.59; N, 2.78. Found: C, 69.23; H, 8.37; N, 2.71. 
14, ${ }^{1} \mathrm{H}$ NMR $\left(500 \mathrm{MHz}\right.$, in $\left.\mathrm{CDCl}_{3}, 25^{\circ} \mathrm{C}\right)$

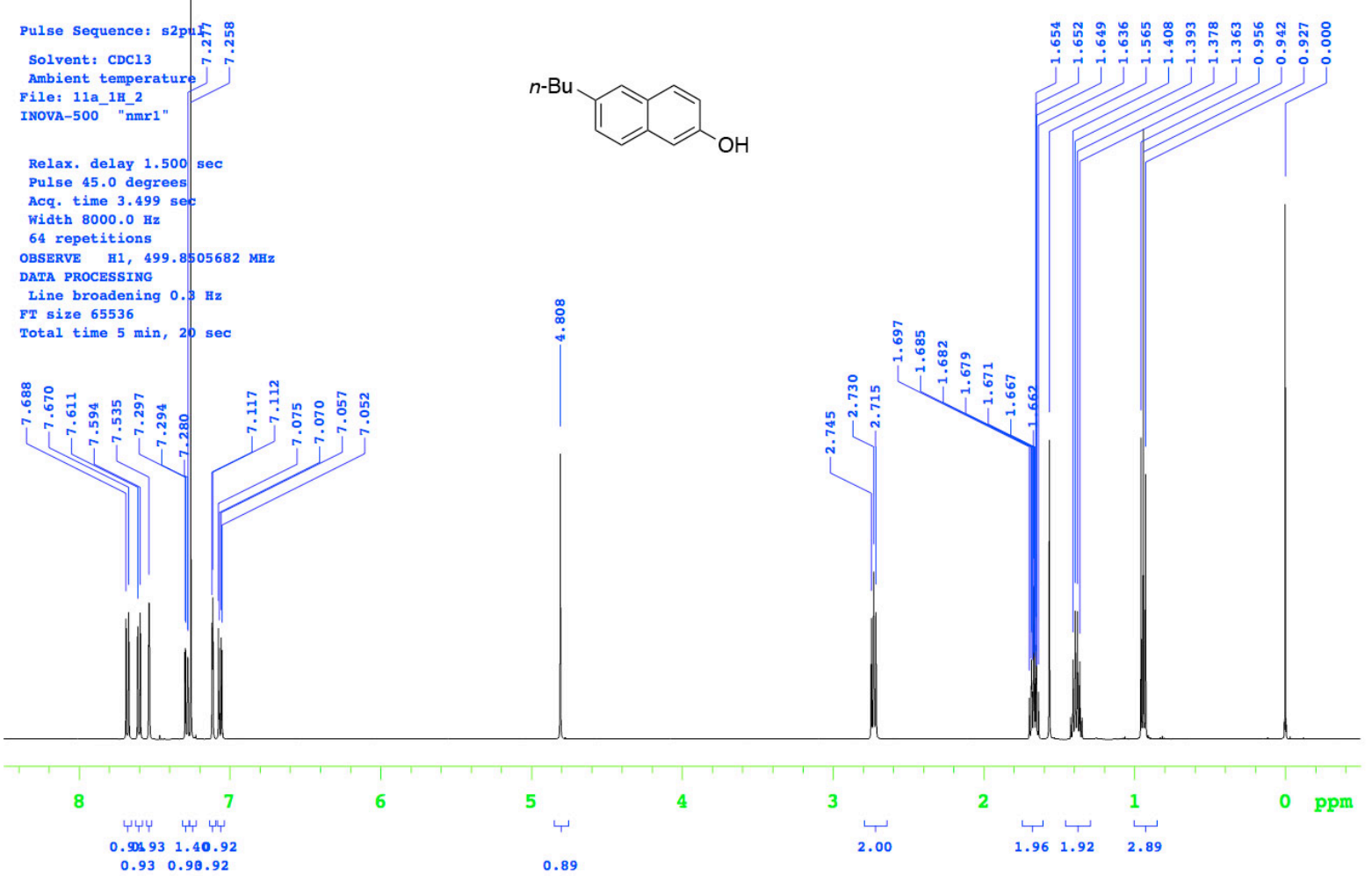

14, ${ }^{13} \mathrm{C}$ NMR $\left(126 \mathrm{MHz}\right.$, in $\left.\mathrm{CDCl}_{3}, 25^{\circ} \mathrm{C}\right)$

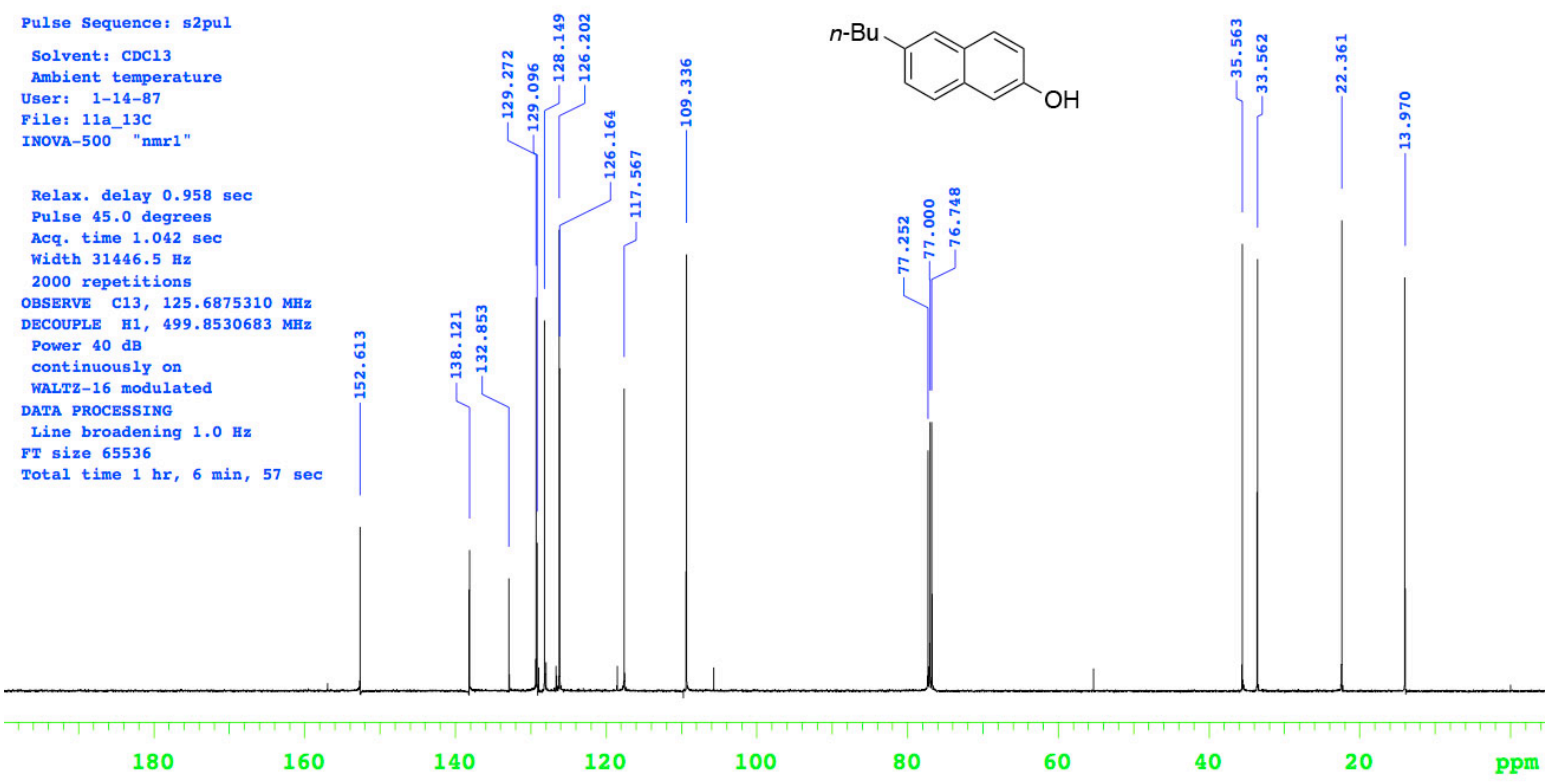


15, ${ }^{1} \mathrm{H}$ NMR $\left(500 \mathrm{MHz}\right.$, in $\left.\mathrm{CDCl}_{3}, 25^{\circ} \mathrm{C}\right)$

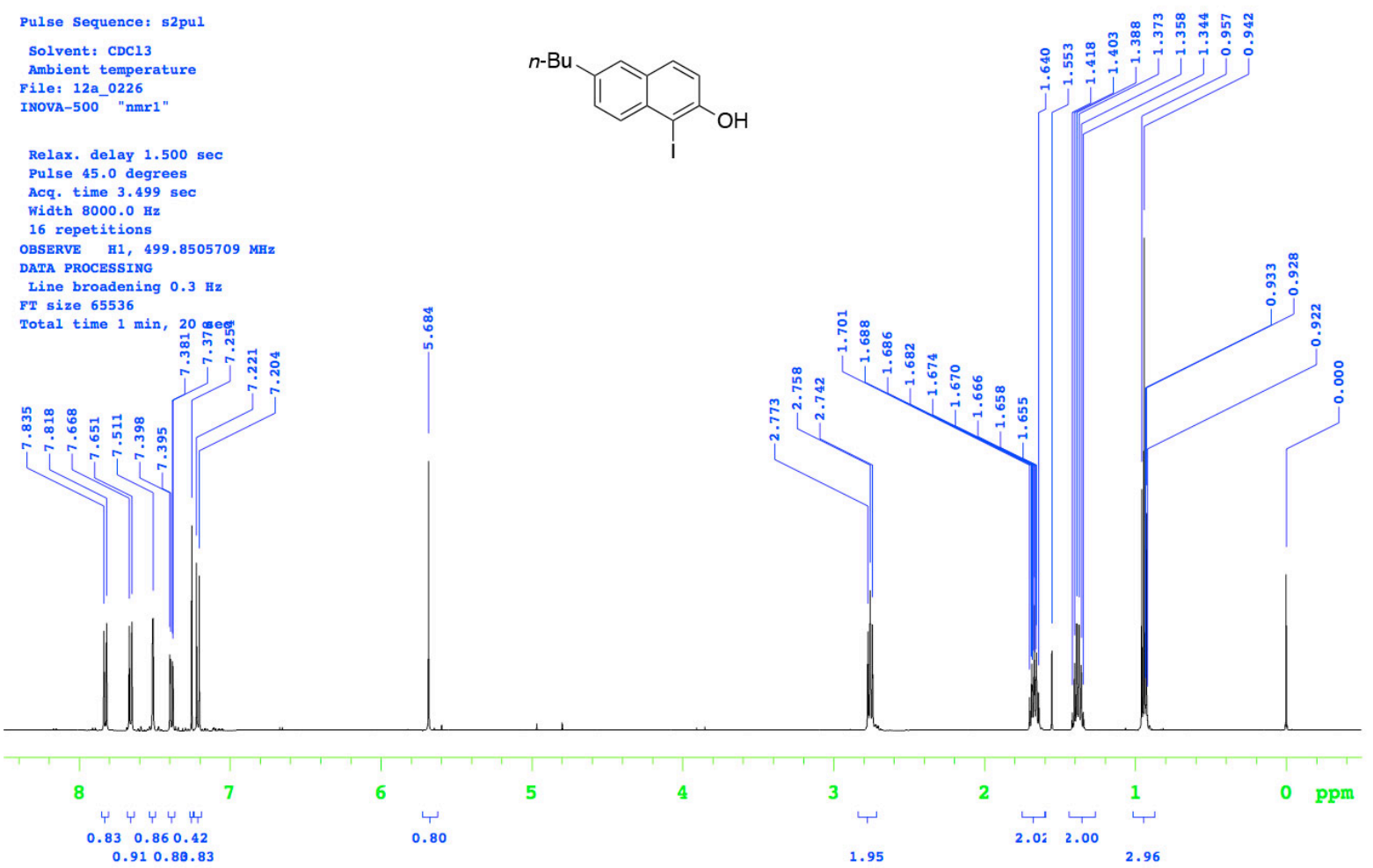

15, ${ }^{13} \mathrm{C}$ NMR $\left(126 \mathrm{MHz}\right.$, in $\left.\mathrm{CDCl}_{3}, 25^{\circ} \mathrm{C}\right)$

Pulse Sequence: s2pul

Solvent: $\mathrm{CDC} 13$

Ambient temperature

User: 1-14-87

File: 12a_0226_13C

INOVA -500 "nmr1"

Relax. delay $0.958 \mathrm{sec}$

Pulse 45.0 degrees

Acq. time $1.042 \mathrm{sec}$

width $31446.5 \mathrm{~Hz}$

4000 repetition

OBSERVE C13, 125.6874945 MH

DECOUPLE H1, 499.8530683 MH

Power $40 \mathrm{~dB}$

continuousiy on

WaLI2-16 modula

DATA PROCESSING

$1.0 \mathrm{Bz}$

Total time $2 \mathrm{hr}, 13 \mathrm{~min}, 55 \mathrm{sec}$
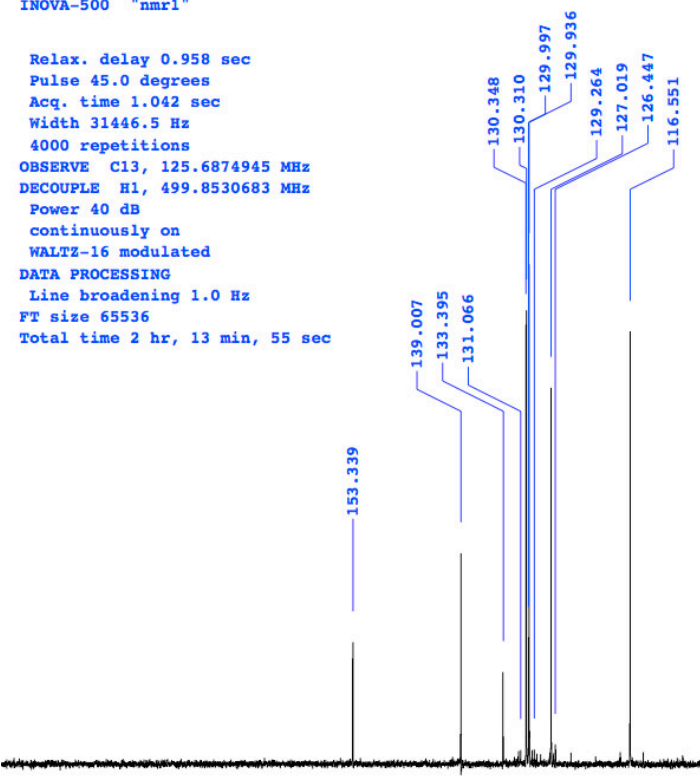

180<smiles>CCCCc1ccc2c(I)c(O)ccc2c1</smiles> 
16, ${ }^{1} \mathrm{H}$ NMR $\left(500 \mathrm{MHz}\right.$, in $\left.\mathrm{CDCl}_{3}, 25^{\circ} \mathrm{C}\right)$

Pulse Sequence: s2pul

Solvent: $\mathrm{CDC} 13$

Ambient temperature

Nova-500 ${ }^{2}$ nmr1

Relax. delay $1.500 \mathrm{sec}$

Pulse 45.0 degrees

Acq. time $3.499 \mathrm{sec}$

32 repetitions

OBSERVE H1, 499.8505714 MHE

DATA PROCESSING

Line broadening $0.3 \mathrm{~Hz}$

T size 65536

Total time $5 \mathrm{~min}, 20 \mathrm{sec}$
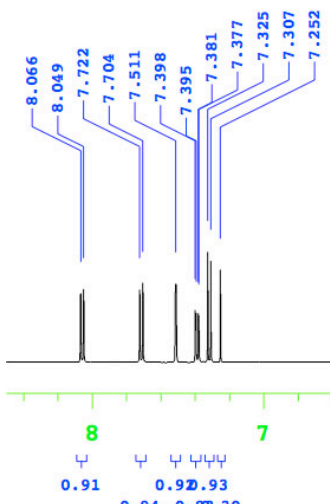

$0.91 \quad 0.92 .93$

6

16, ${ }^{13} \mathrm{C}$ NMR $\left(126 \mathrm{MHz}\right.$, in $\left.\mathrm{CDCl}_{3}, 25^{\circ} \mathrm{C}\right)$

Pulse Sequence: s2pul

Solvent: CDC13

Temp. $25.0 \mathrm{C} / 298.1 \mathrm{~K}$

User: $1-14-87$

File: 13a_13C

INOVA- 500 " nmr 1"

Relax. delay $0.958 \mathrm{sec}$

Pulse 45.0 degrees

Acq. time $1.042 \mathrm{sec}$

width 31446.5 Hz

000 repetitions

BSERVE C13, 125.6875249 MH

DECOUPLE H1, 499.8530683 MH

Power $40 \mathrm{~dB}$

continuous $I_{Y}$ on

DATA PROCESSING

Total time $1 \mathrm{hr}, 6 \mathrm{~min}, 57 \mathrm{sec}$

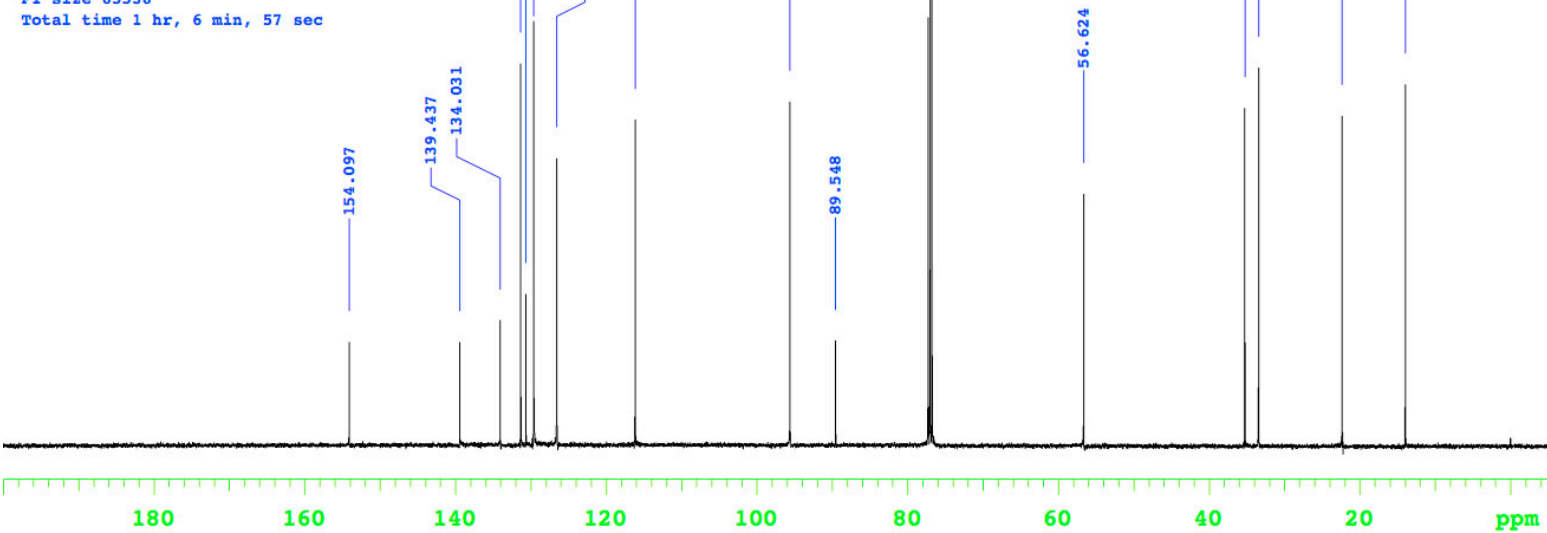


17b, ${ }^{1} \mathrm{H}$ NMR $\left(500 \mathrm{MHz}\right.$, in $\left.\mathrm{CDCl}_{3}, 25^{\circ} \mathrm{C}\right)$

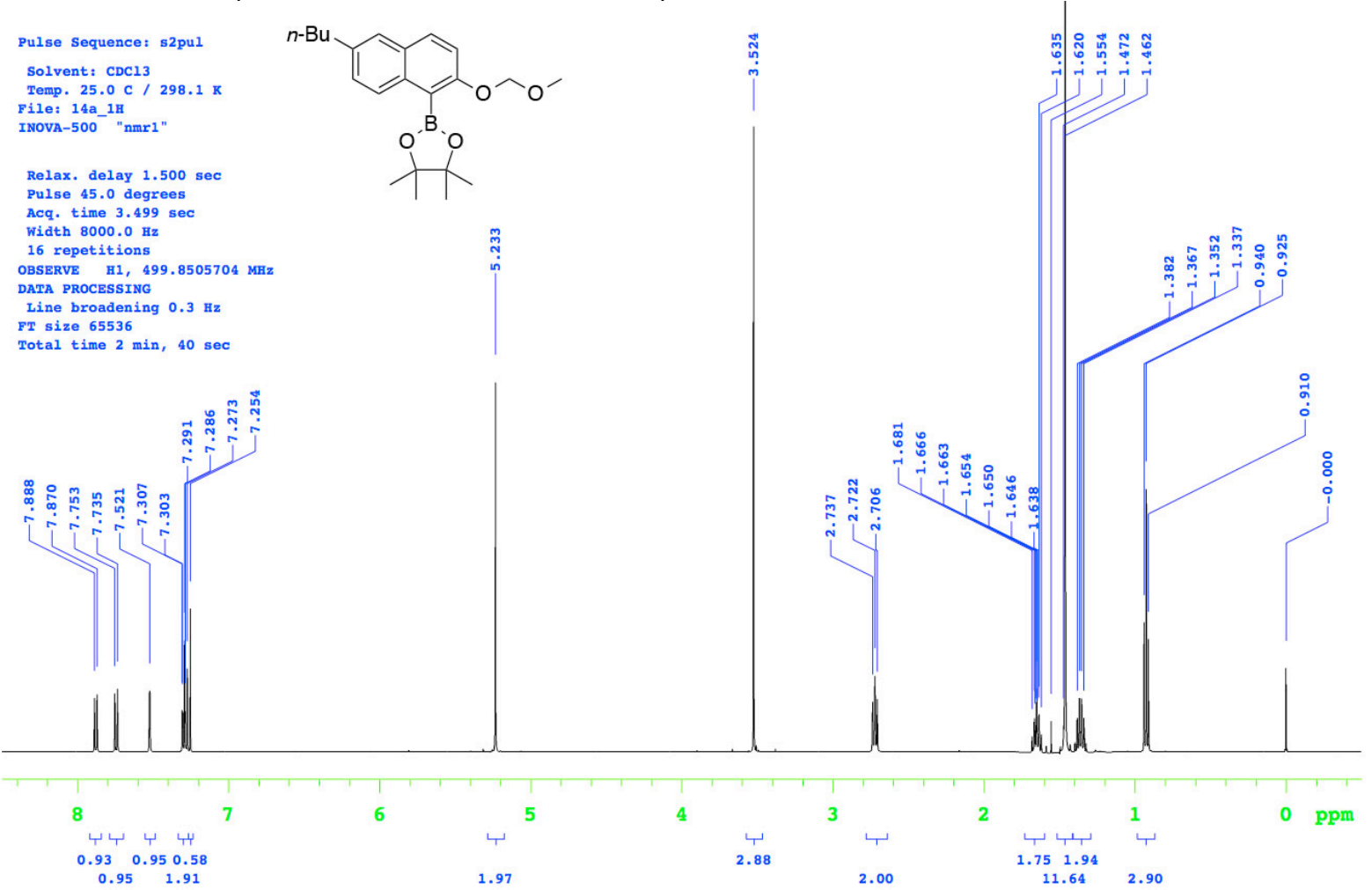

17b, ${ }^{13} \mathrm{C}$ NMR $\left(126 \mathrm{MHz}\right.$, in $\left.\mathrm{CDCl}_{3}, 25^{\circ} \mathrm{C}\right)$

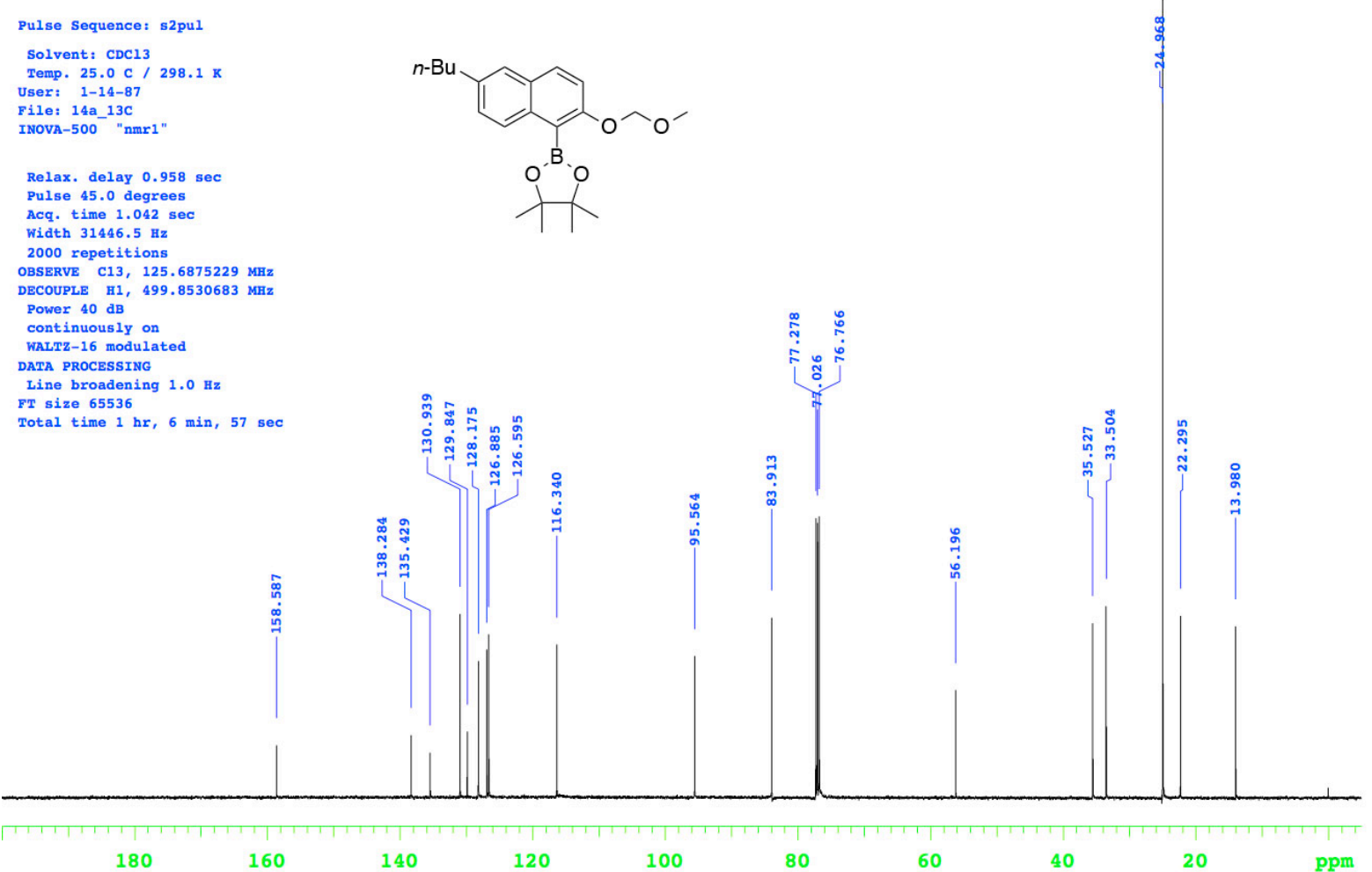


18, ${ }^{1} \mathrm{H}$ NMR $\left(500 \mathrm{MHz}\right.$, in $\left.\mathrm{CDCl}_{3}, 25^{\circ} \mathrm{C}\right)$

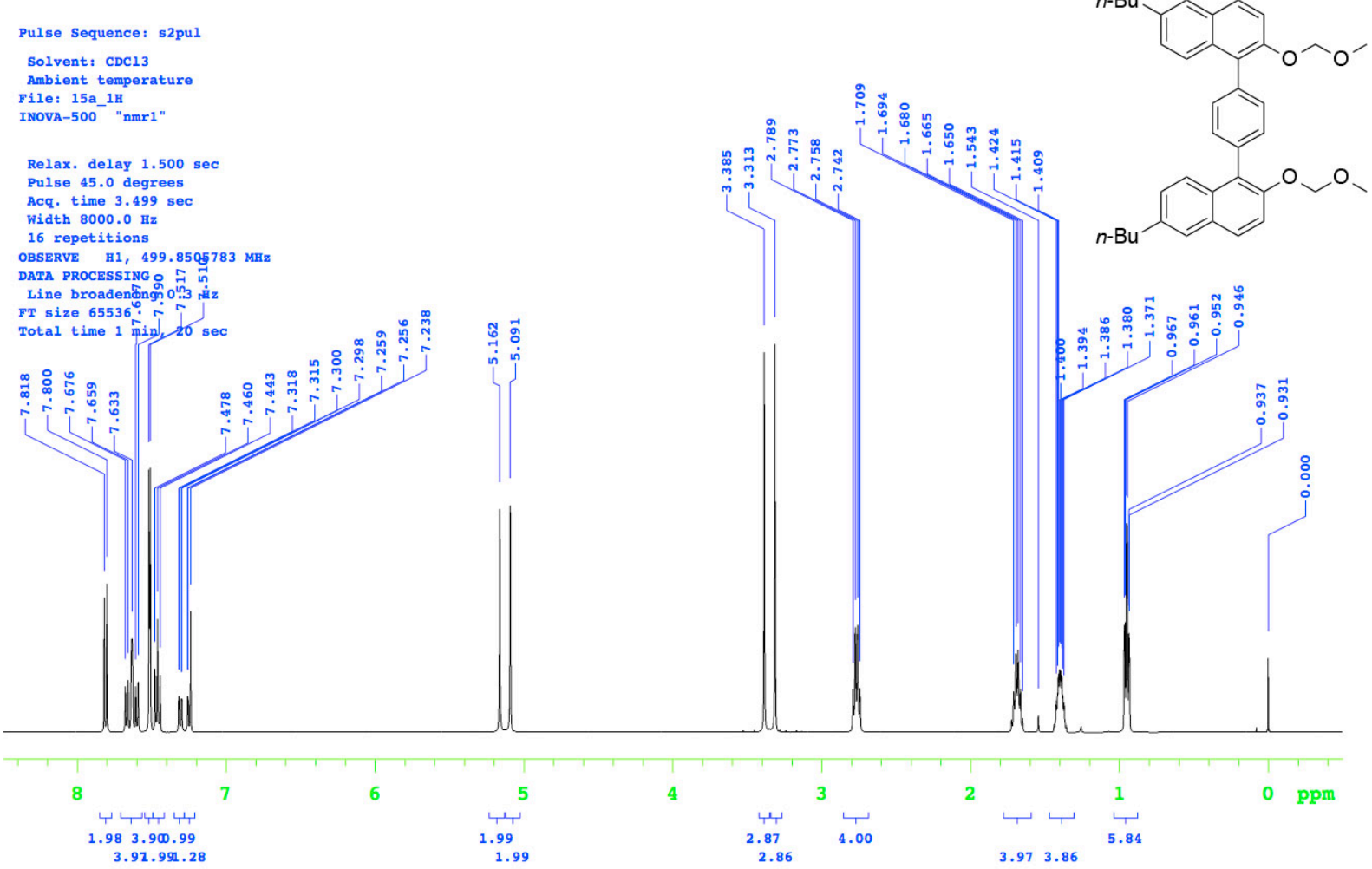

18, ${ }^{13} \mathrm{C}$ NMR $\left(126 \mathrm{MHz}\right.$, in $\left.\mathrm{CDCl}_{3}, 25^{\circ} \mathrm{C}\right)$

Pulse Sequence: s2pul

Solvent: $\mathrm{CDC} 13$

Ambient temperature

User: 1-14-87

File: $15 a_{-} 13 \mathrm{C}$
INova-500 "nmr1"

Relax. delay $0.958 \mathrm{sec}$ Pulse 45.0 degrees

Acq. time 1.042 sec

Width $31446.5 \mathrm{~Hz}$

2000 repetitions

DECRE C13, 125.6875290 MHE

Power $40 \mathrm{~dB}$

WaLTz-16 modulated

DATA PROCESSING
Line broadening $1.0 \mathrm{~Hz}$

Line broadening $1.0 \mathrm{~Hz}$
$\mathrm{FT}$ size 65536

1

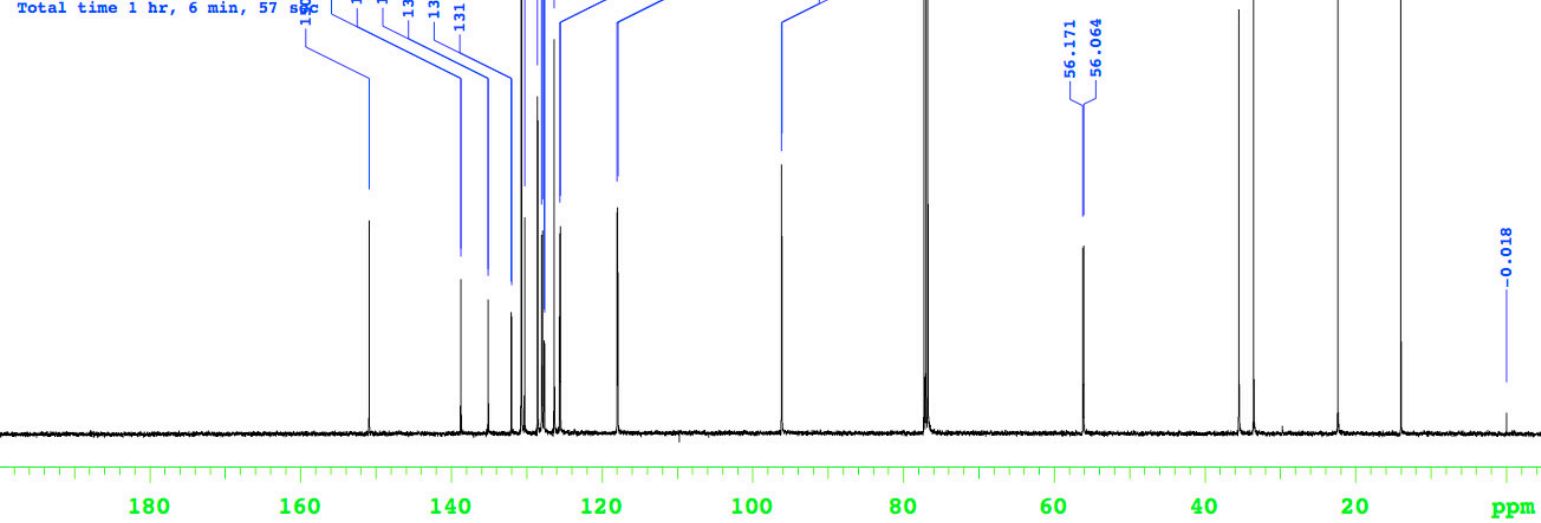


1b, ${ }^{1} \mathrm{H}$ NMR $\left(500 \mathrm{MHz}\right.$, in $\left.\mathrm{CDCl}_{3}, 25^{\circ} \mathrm{C}\right)$

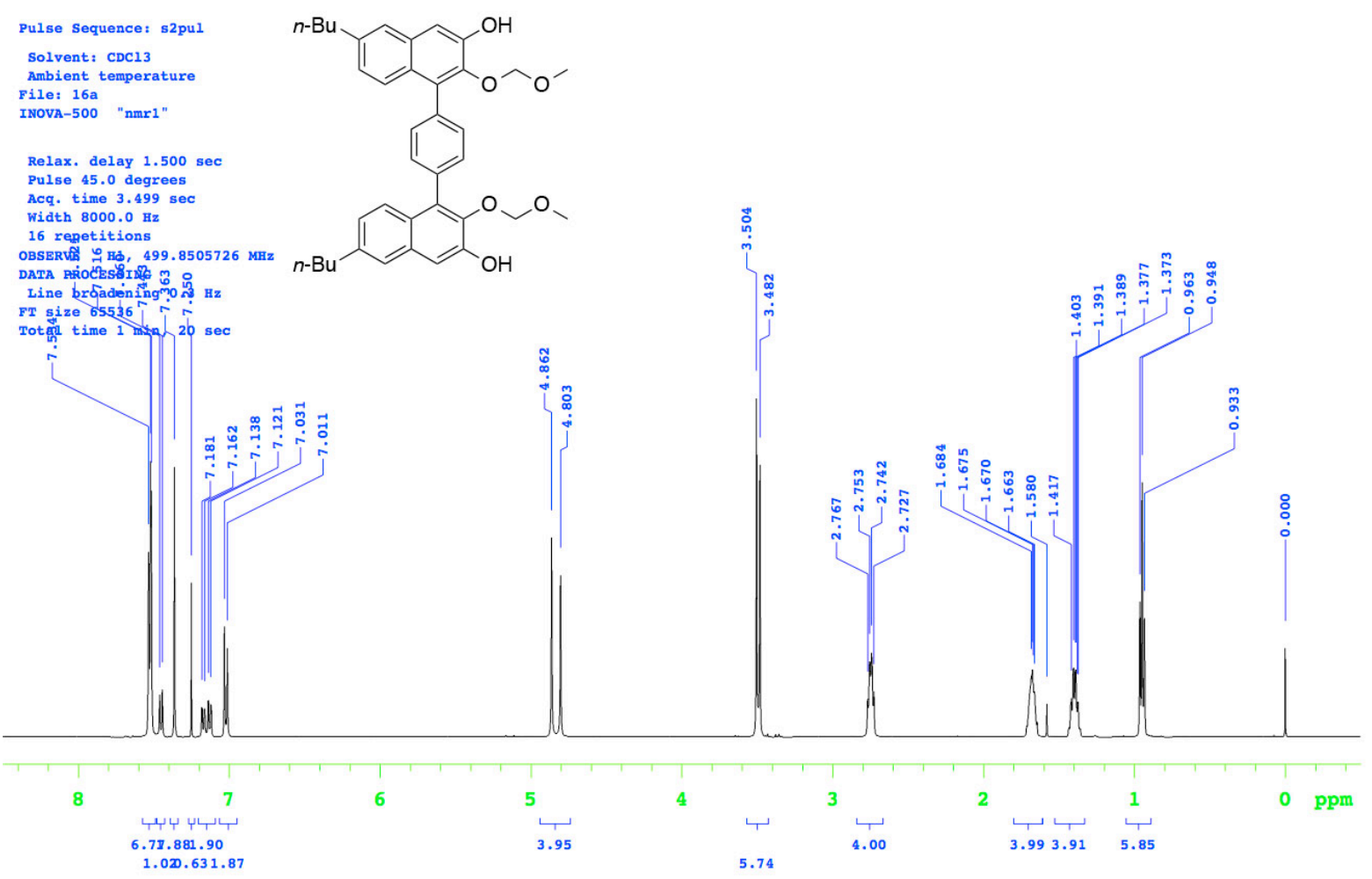

1b, ${ }^{13} \mathrm{C}$ NMR $\left(126 \mathrm{MHz}\right.$, in $\left.\mathrm{CDCl}_{3}, 25^{\circ} \mathrm{C}\right)$

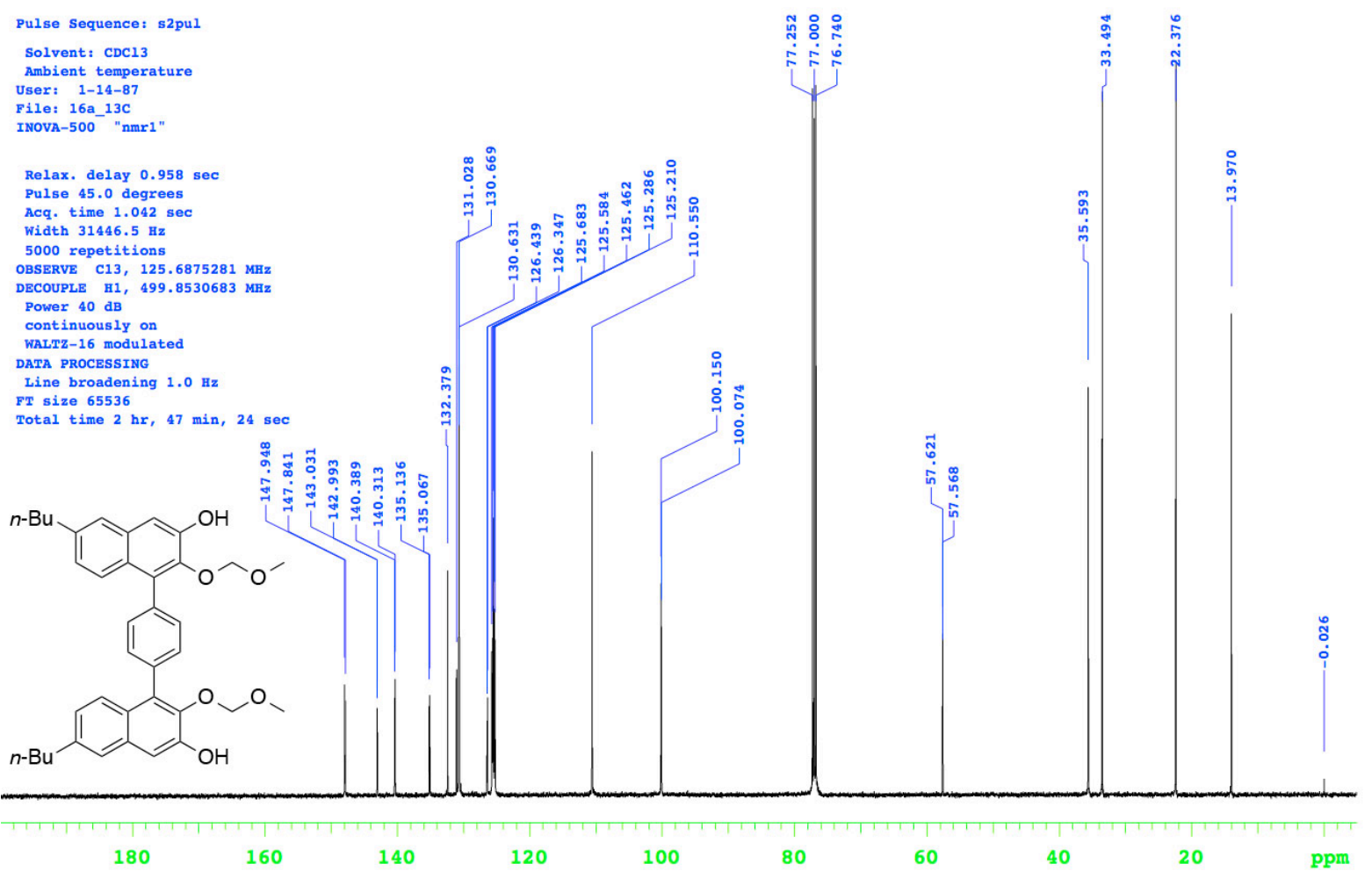


19a, ${ }^{1} \mathrm{H}$ NMR $\left(500 \mathrm{MHz}\right.$, in $\left.\mathrm{CDCl}_{3}, 25^{\circ} \mathrm{C}\right)$

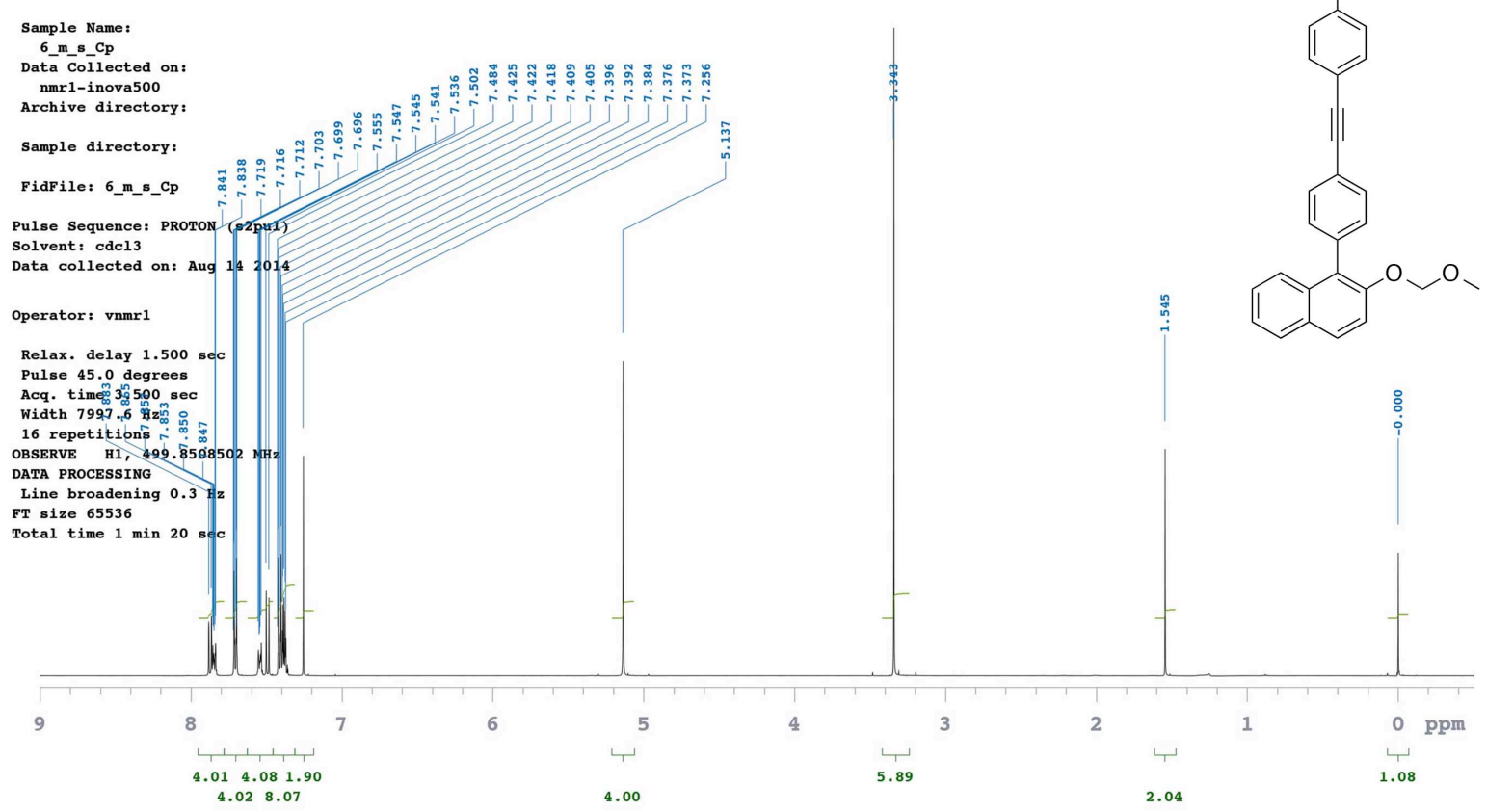

19a, ${ }^{13} \mathrm{C}$ NMR $\left(126 \mathrm{MHz}\right.$, in $\left.\mathrm{CDCl}_{3}, 25^{\circ} \mathrm{C}\right)$

Sample Name:
6_m_s_Cp
Data Collected on:
nmr1-inova500
Archive directory:
Sample directory:
Fidfile: 6_m_s_Cp_13C

Pulse Sequence: CARBON (s2pul)

on: Aug 142014

Operator: vnmr

Relax. delay $1.957 \mathrm{sec}$ Pulse 45.0 degrees Acq. time $1.043 \mathrm{~s}$ 2368 repetition

OBSERVE C13, 125.6875708 MH

DECOUPLE H1，499.8533353 MH

Power $40 \mathrm{~dB}$

continuously on

WALTZ-16 modulated

DATA PROCESSING

Line broadening $1.0 \mathrm{~Hz}$

Total time $2 \mathrm{hr}, 30 \mathrm{~min}$

180

160

140

100

80

40

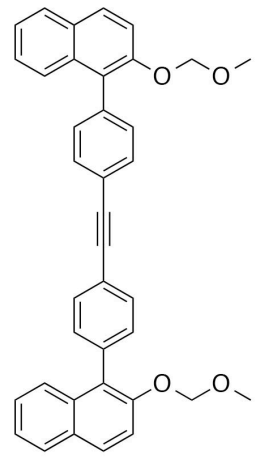


19b, ${ }^{1} \mathrm{H}$ NMR $\left(500 \mathrm{MHz}\right.$, in $\left.\mathrm{CDCl}_{3}, 25^{\circ} \mathrm{C}\right)$

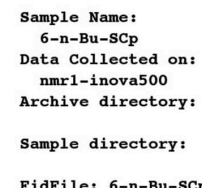

FidFile: $6-n-B u-S C p-1 H$

Pulse Sequence: PROTON (s2pul)

Solvent: cdc13

172019 工

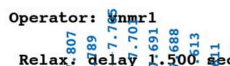

Pulse 45.0 degrees

Acq. time 3.500 se

Width $7997.6 \mathrm{~Hz}$

16 repetitions

OBSERVE H1,

DATA PROCESSING

FT size 65536

tal time $1 \mathrm{~min} 20 \mathrm{sec}$

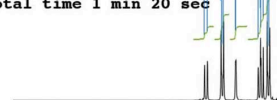

$9 \quad 8$

8

$2.12 .15 \quad 4.32$
4.098 .34

2.12 .15
4.098 .34

19b, ${ }^{13} \mathrm{C}$ NMR $\left(126 \mathrm{MHz}\right.$, in $\left.\mathrm{CDCl}_{3}, 25^{\circ} \mathrm{C}\right)$

Sample Name:

Data Collected on:

nmr1-inova500

Sample directory

FidFile: 6-n-Bu-SCp-13C

Pulse Sequence: CARBON (s2pul)

Data collected on: Nov 172014

Operator: vnmr1

Relax. delay $1.957 \mathrm{sec}$ Pulse 45.0 degrees

Acq. time $1.043 \mathrm{sec}$

width $31421.8 \mathrm{~Hz}$

5000 repetition

OBSERVE C13，125.6875714 MH

DECOUPLE H1，499.8557096 MH

Power $40 \mathrm{~dB}$

continuousiy on

Tra

Line broadening $1.0 \mathrm{~Hz}$

FT size 65536

Total time $4 \mathrm{hr}, 10 \mathrm{~min}$
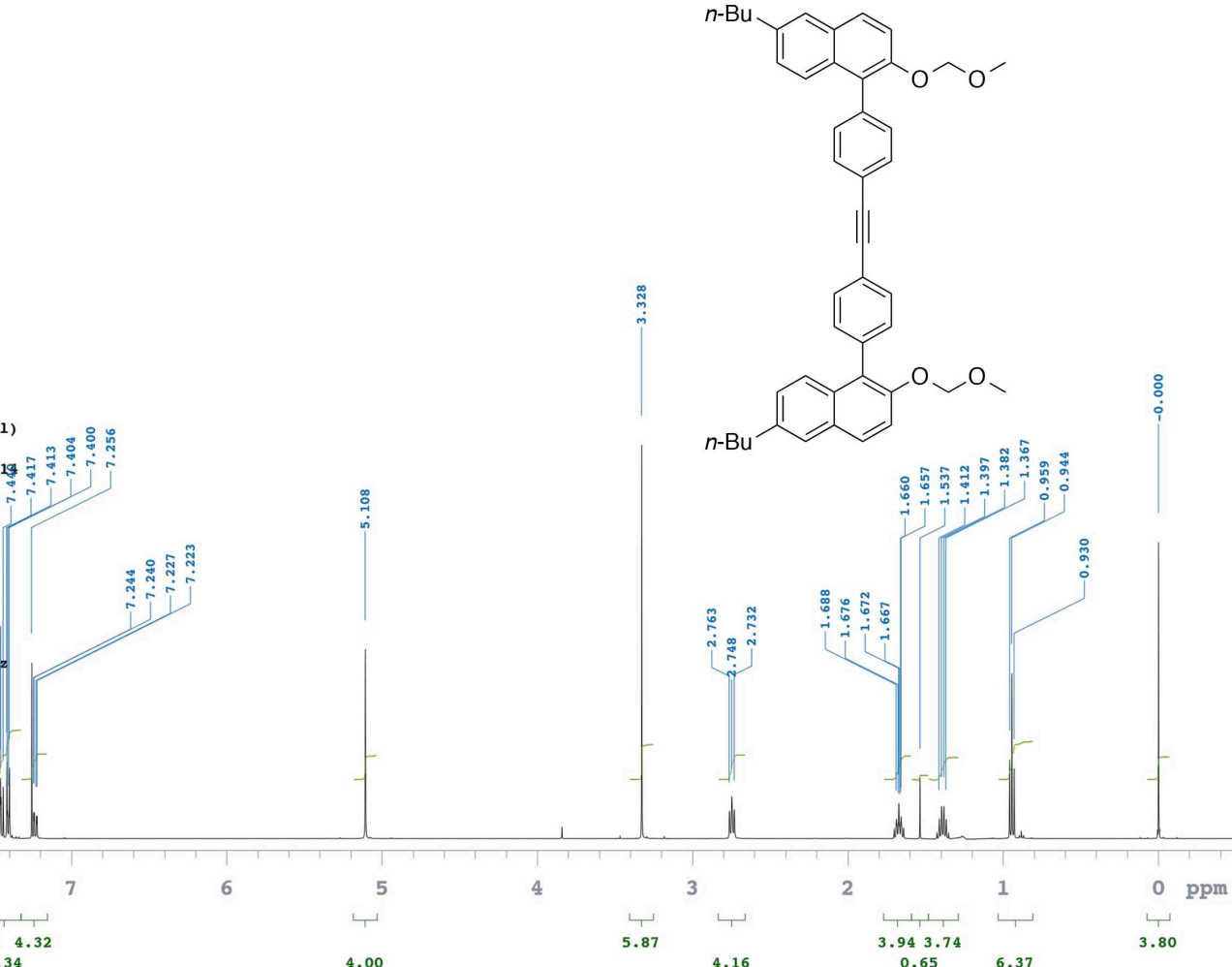
2a, ${ }^{1} \mathrm{H}$ NMR $\left(500 \mathrm{MHz}\right.$, in $\left.\mathrm{CDCl}_{3}, 25^{\circ} \mathrm{C}\right)$

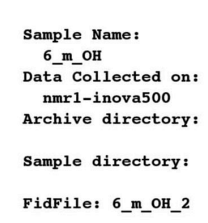

Pulse Sequence: PROTON (s2pul)

Solvent: cdc13

n: Ju1 252014

Operator: vnmr1

Relax. delay $1.500 \mathrm{sec}$

Pulse 45.0 degrees

Acq. time 3.500 sec

Width $7997.6 \mathrm{HZ}$

16 repetitions
OBSERVE H1, 499.8508499 MH

DSERe Hi,

Line broadening $0.3 \mathrm{~Hz}$

FT size 65536
Total time 1 min $20 \mathrm{sec}$
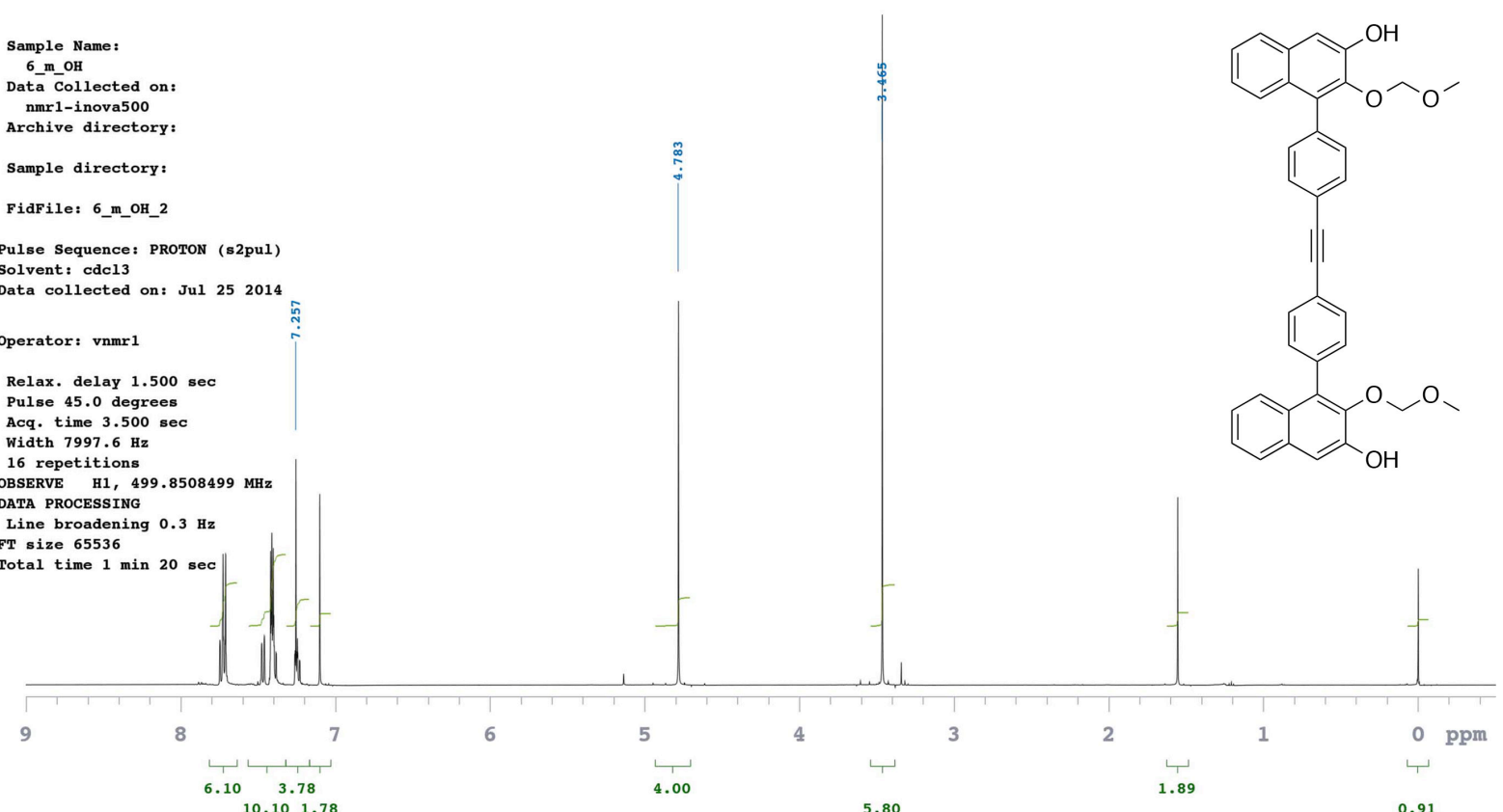

2a, ${ }^{13} \mathrm{C}$ NMR $\left(126 \mathrm{MHz}\right.$, in $\left.\mathrm{CDCl}_{3}, 25^{\circ} \mathrm{C}\right)$

Pulse Sequence: CARBoN (s2pul)

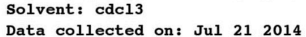

operator: vnmr1

Relax. delay $1.957 \mathrm{sec}$

Pulse 45.0 degrees

Acq. time $1.043 \mathrm{se}$

Width $31421.8 \mathrm{~Hz}$

1000 repetitions

OBSERVE C13，125.6875853 MHz

DECOUPLE H1, 499.8533353 MH

Power $40 \mathrm{~dB}$

continuousiy on

(a)

Line brocsing 1.0

FT size 65536

Total time $50 \mathrm{~min}$

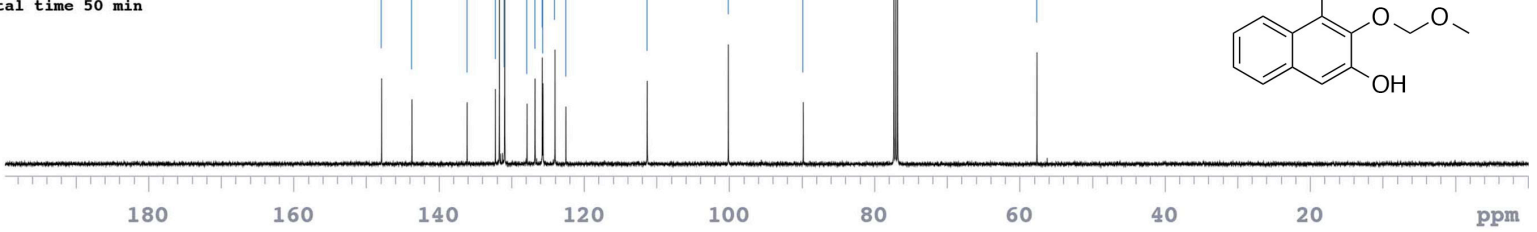


2b, ${ }^{1} \mathrm{H}$ NMR (500 MHz, in $\left.\mathrm{CDCl}_{3}, 25^{\circ} \mathrm{C}\right)$

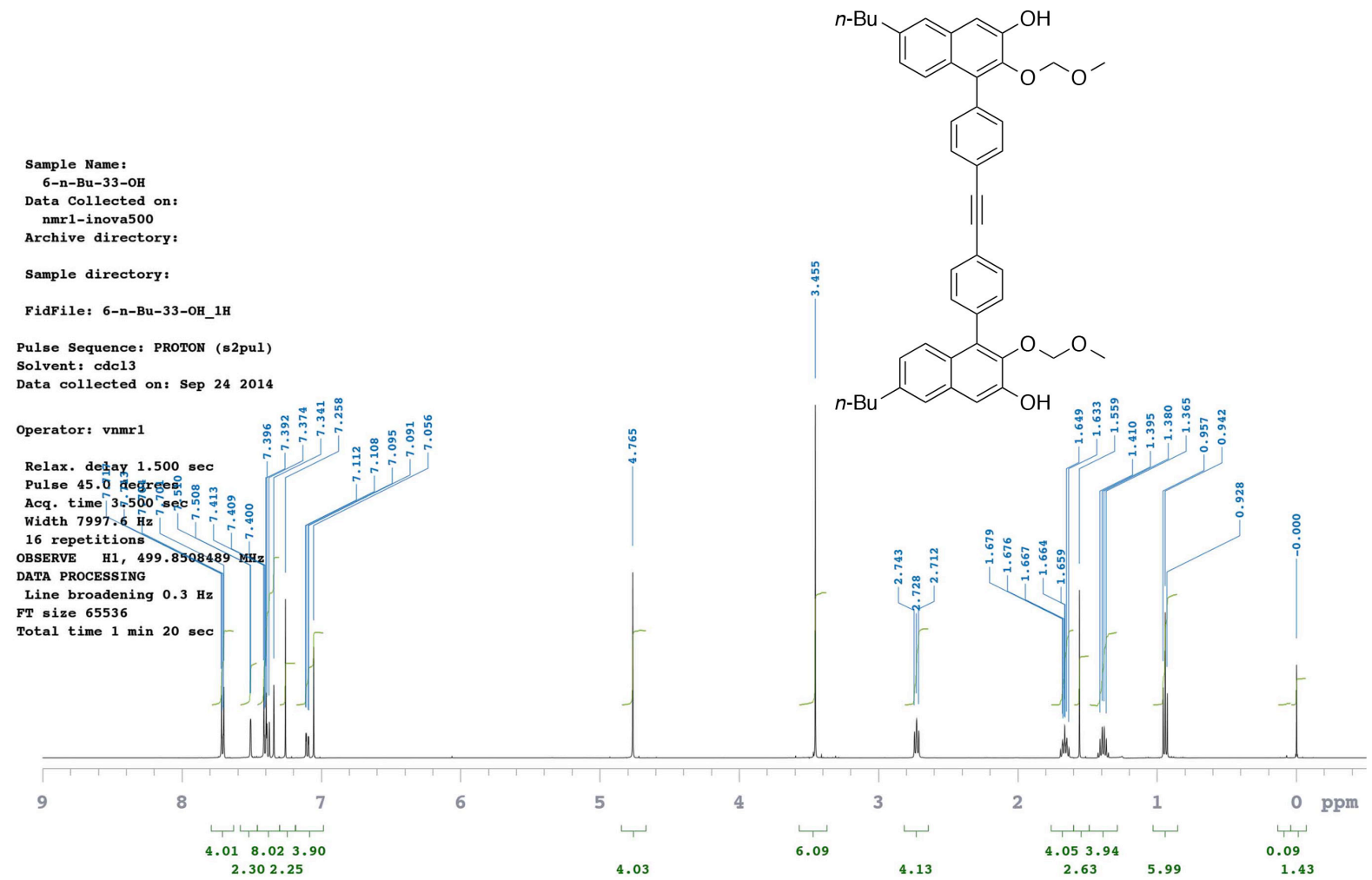

2b, ${ }^{13} \mathrm{C}$ NMR $\left(126 \mathrm{MHz}\right.$, in $\left.\mathrm{CDCl}_{3}, 25^{\circ} \mathrm{C}\right)$
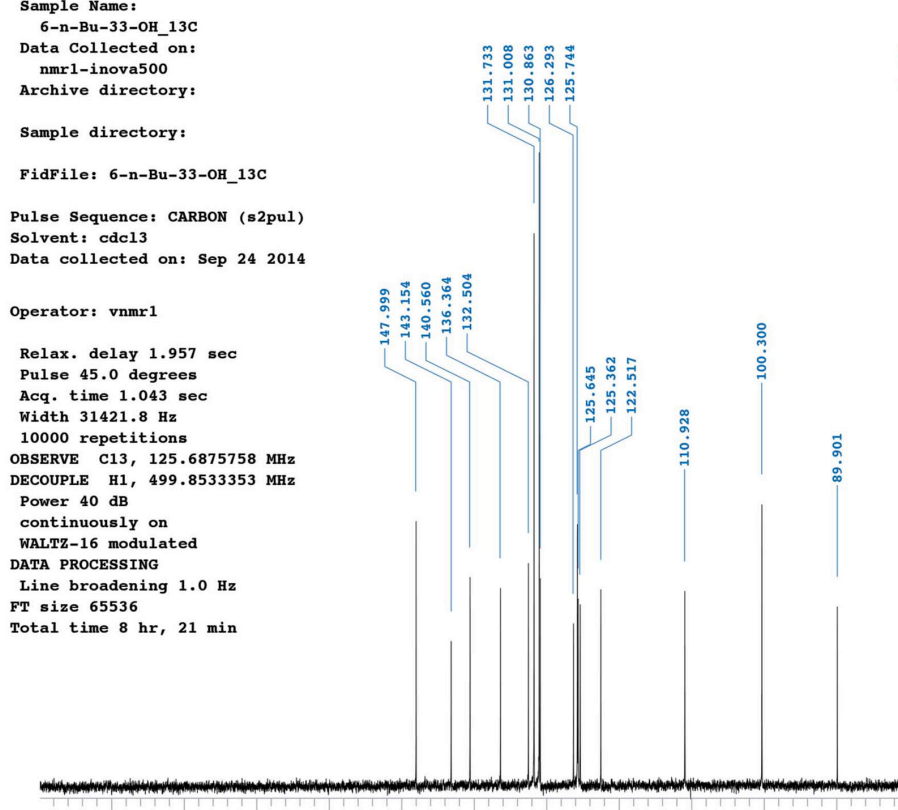
4a. $\left(\mathrm{Me}_{2} \mathrm{NH}_{2}\right)_{4},{ }^{1} \mathrm{H}$ NMR $\left(500 \mathrm{MHz}\right.$, in DMSO- $\left.d_{6}, 25^{\circ} \mathrm{C}\right)$

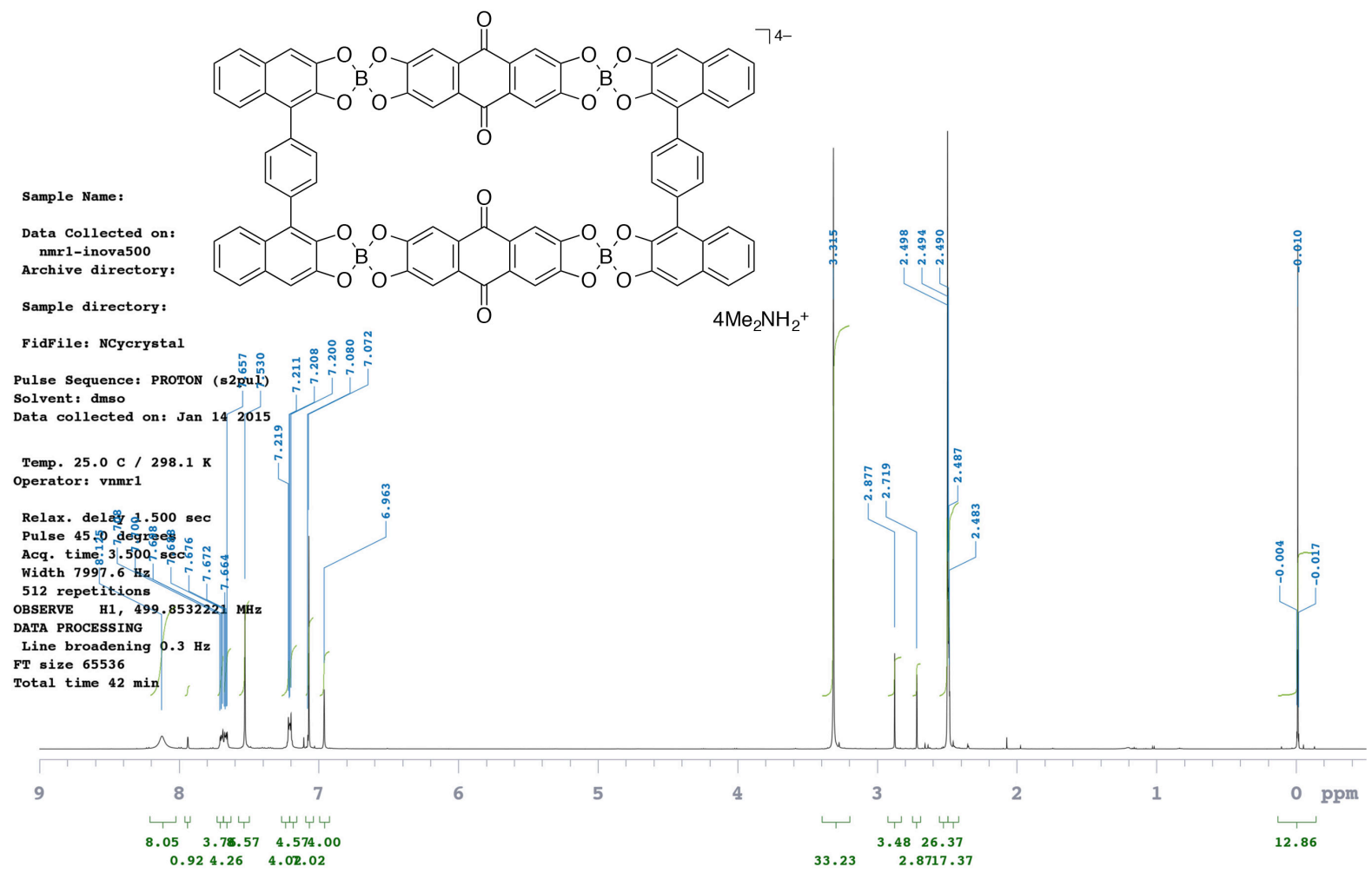

4a. $\left(\mathrm{Me}_{2} \mathrm{NH}_{2}\right)_{4},{ }^{13} \mathrm{C}$ NMR $\left(126 \mathrm{MHz}\right.$, in DMSO- $\left.d_{6}, 25^{\circ} \mathrm{C}\right)$

Sample Name:

Data Collected on: nmr1-inova500

Sample directory :

Fidfile: NCycrystal_13C

Pulse Sequence: CARBoN (s2pul)

Solvent: dmso
Data collected on: Jan 142015

Temp. $25.0 \mathrm{C} / 298.1 \mathrm{~K}$

operator: vnmr

Relax. delay $1.957 \mathrm{sec}$ Pulse 45.0 degrees Acq. time $1.043 \mathrm{sec}$

20000 repetition

OBSERVE C13

DECOUPLE H1 « 499.8557096 MH

Power $40 \mathrm{~dB}-1$

continuously on

WALTZ-16 modulat

DATA PROCESSING

Line broadening $1.0 \mathrm{~Hz}$

Total time $16 \mathrm{hr}, 42 \mathrm{~min}$

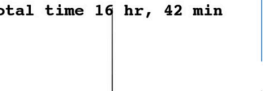

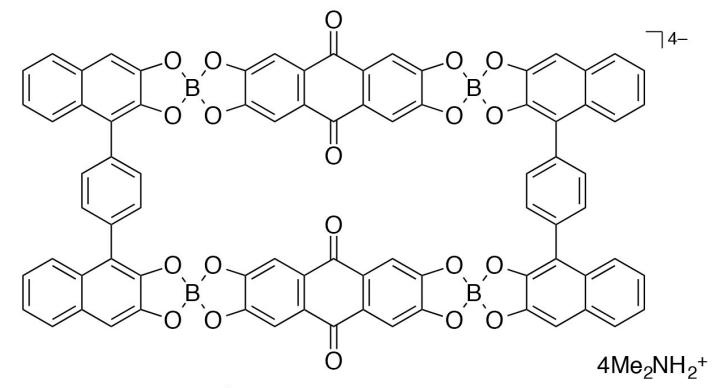

$4 \mathrm{Me}_{2} \mathrm{NH}_{2}{ }^{+}$

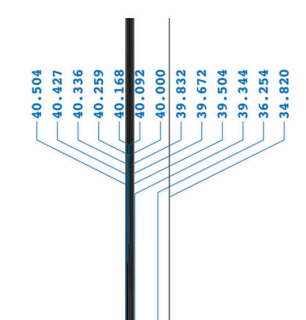

\section{مึ:}

ㄷำ

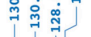

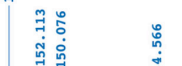

苞

J

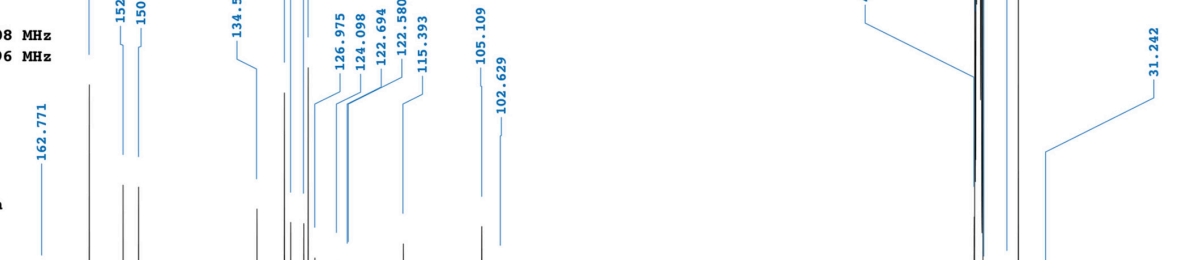

ฺัต 
4b. $\left(\mathrm{Me}_{2} \mathrm{NH}_{2}\right)_{4},{ }^{1} \mathrm{H} \mathrm{NMR}\left(500 \mathrm{MHz}\right.$, in DMSO- $\left.d_{6}, 25^{\circ} \mathrm{C}\right)$
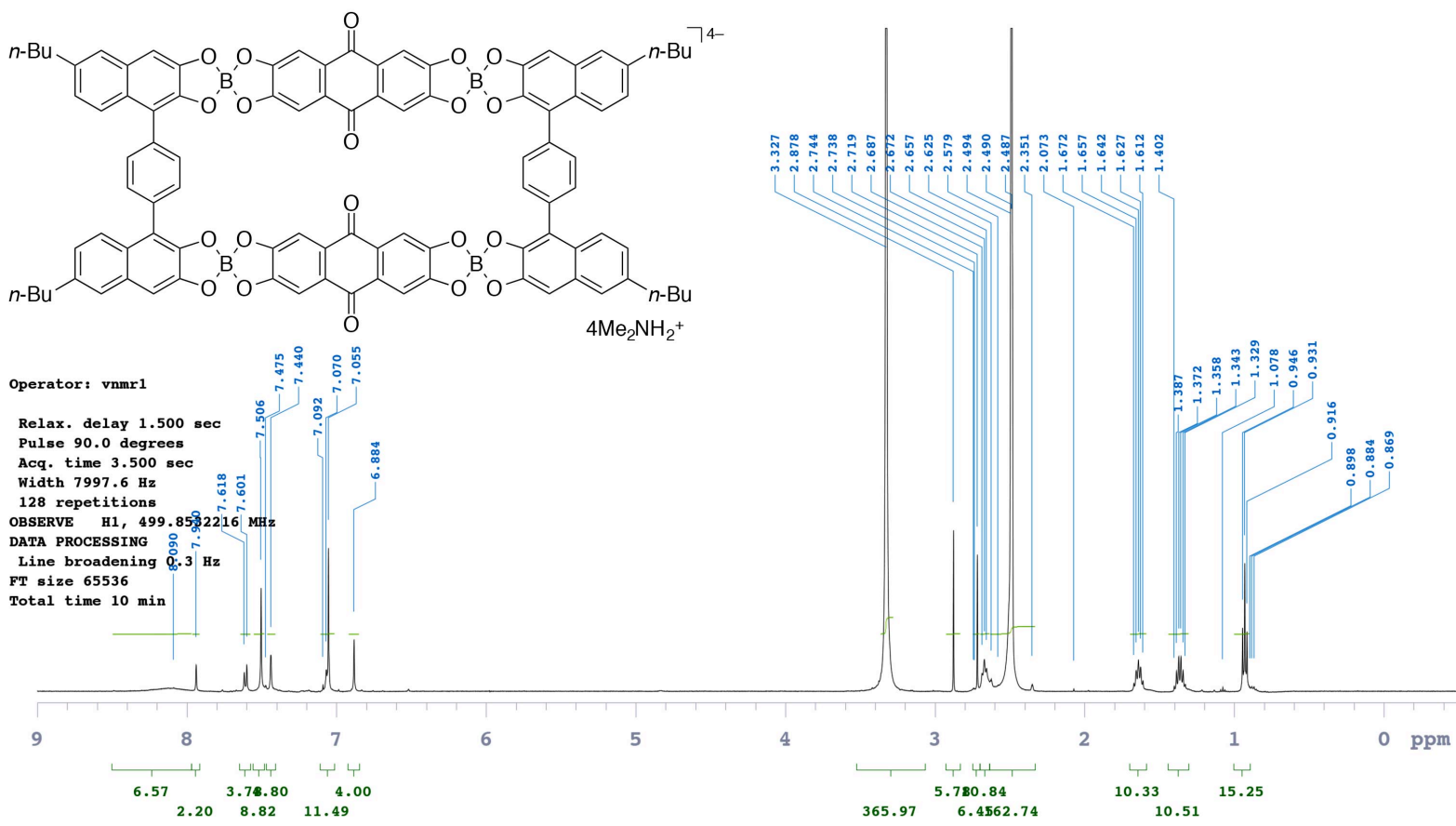

4b $\left(\mathrm{Me}_{2} \mathrm{NH}_{2}\right){ }_{4},{ }^{13} \mathrm{C}$ NMR $\left(126 \mathrm{MHz}\right.$, in DMSO- $\left.d_{6}, 25^{\circ} \mathrm{C}\right)$

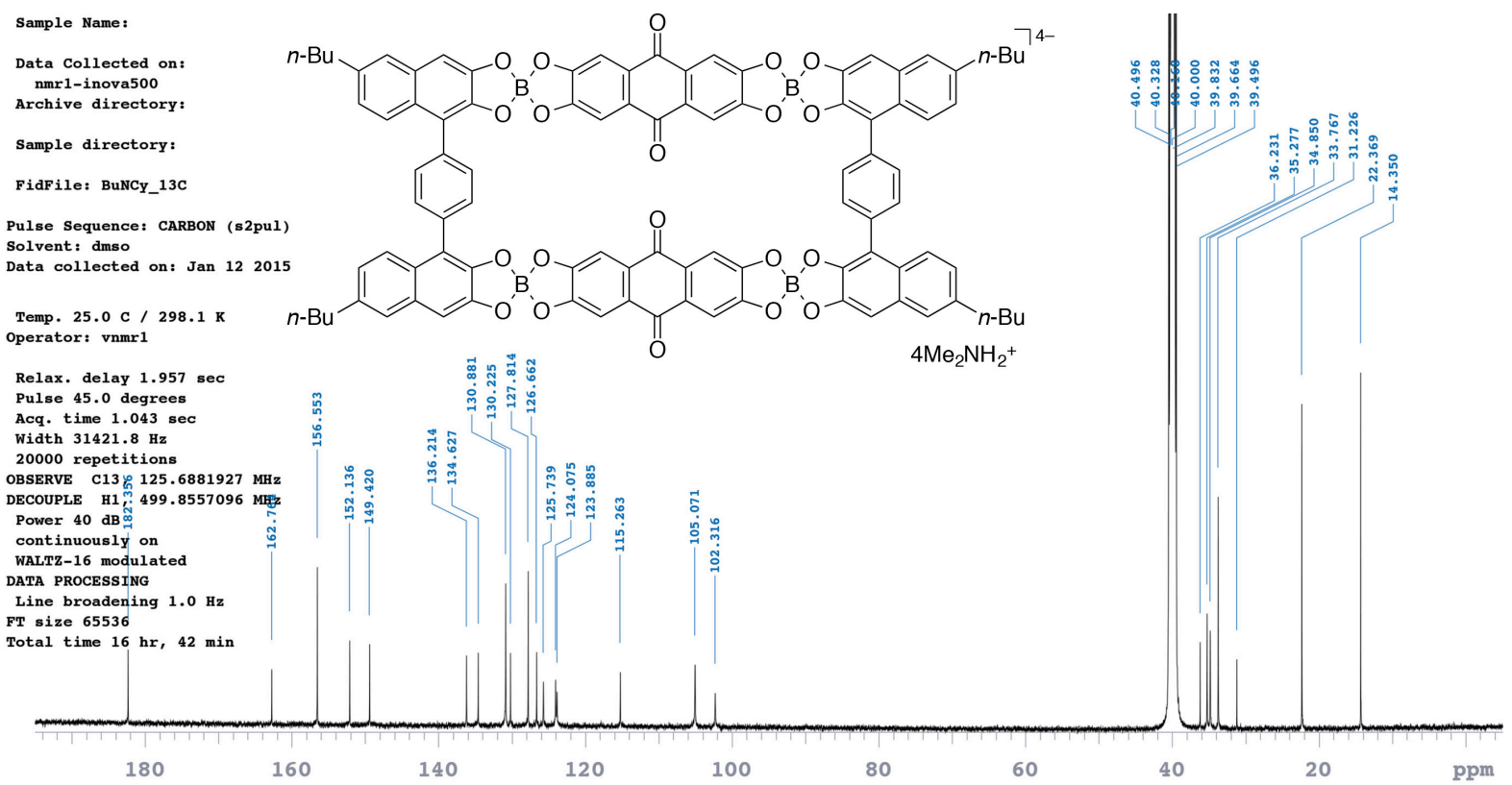


5a.(TBA $)_{4},{ }^{1} \mathrm{H}$ NMR $\left(500 \mathrm{MHz}\right.$, in DMSO- $\left.d_{6}, 25^{\circ} \mathrm{C}\right)$

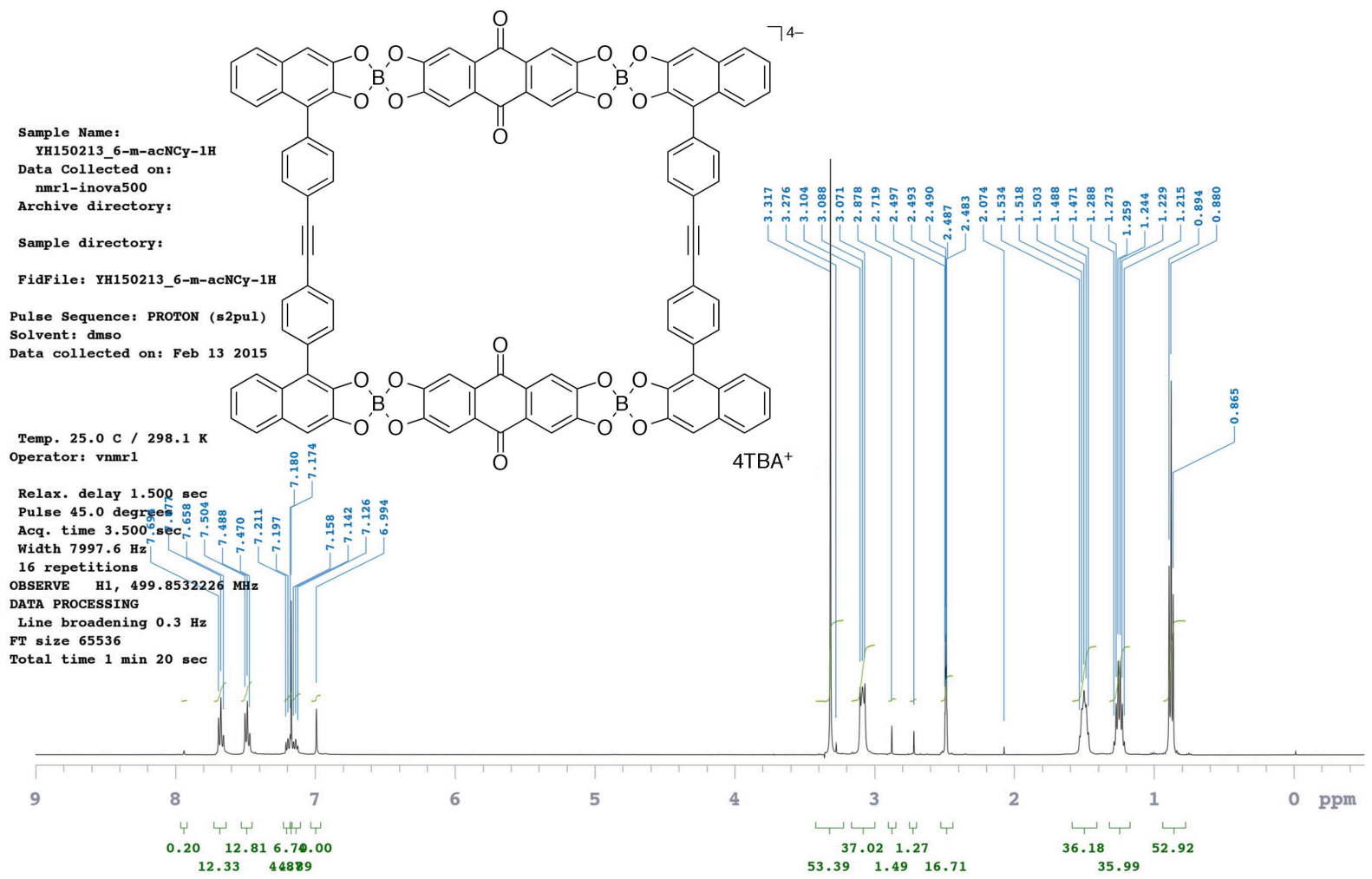

5a.(TBA $)_{4},{ }^{13} \mathrm{C}$ NMR $\left(126 \mathrm{MHz}\right.$, in DMSO- $\left.d_{6}, 25^{\circ} \mathrm{C}\right)$

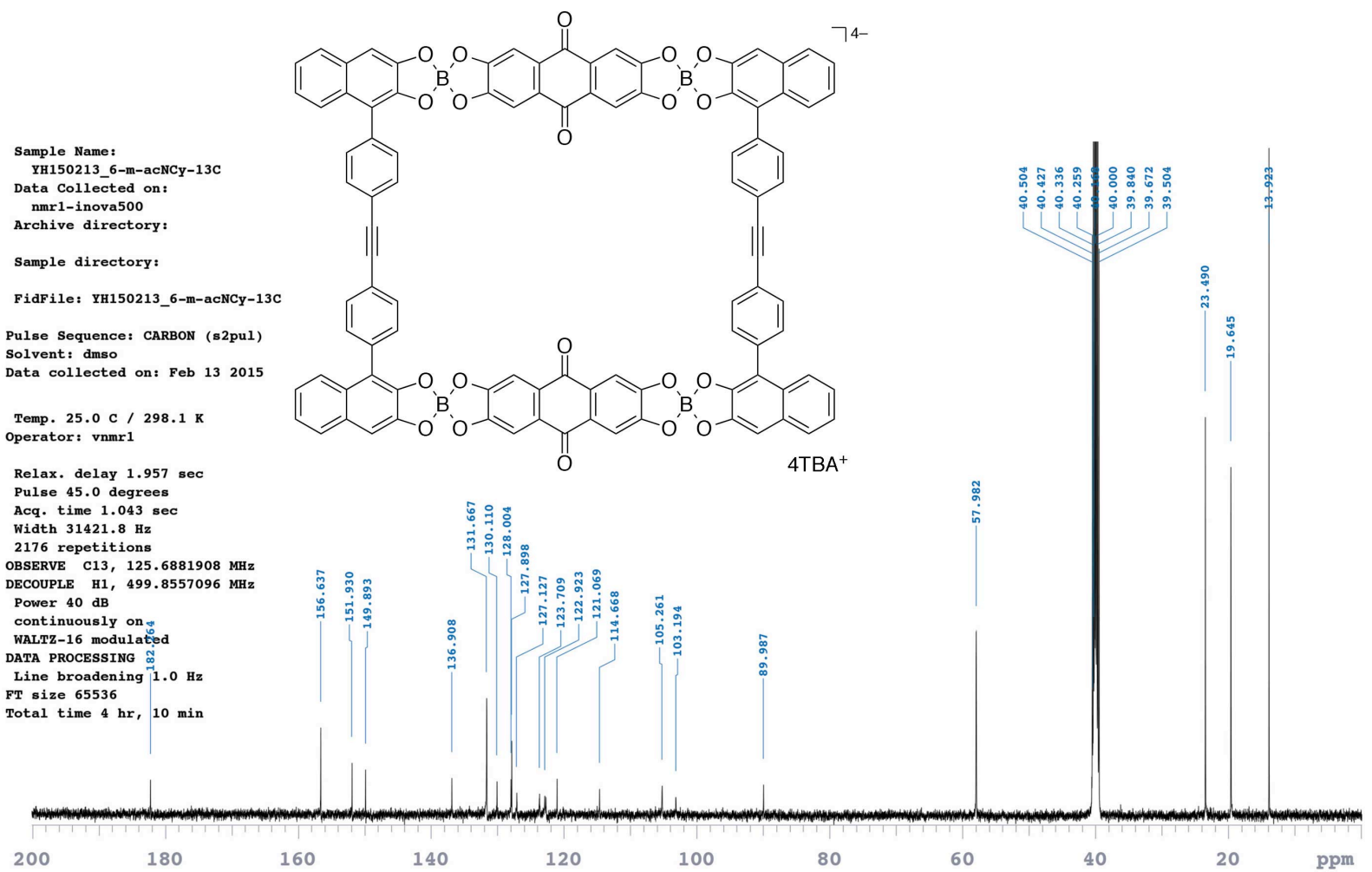


Supporting Information

sb $\left(\mathrm{Me}_{2} \mathrm{NH}_{2}\right)_{4},{ }^{1} \mathrm{H}$ NMR $\left(500 \mathrm{MHz}\right.$, in DMSO- $\left.d_{6}, 25^{\circ} \mathrm{C}\right)$

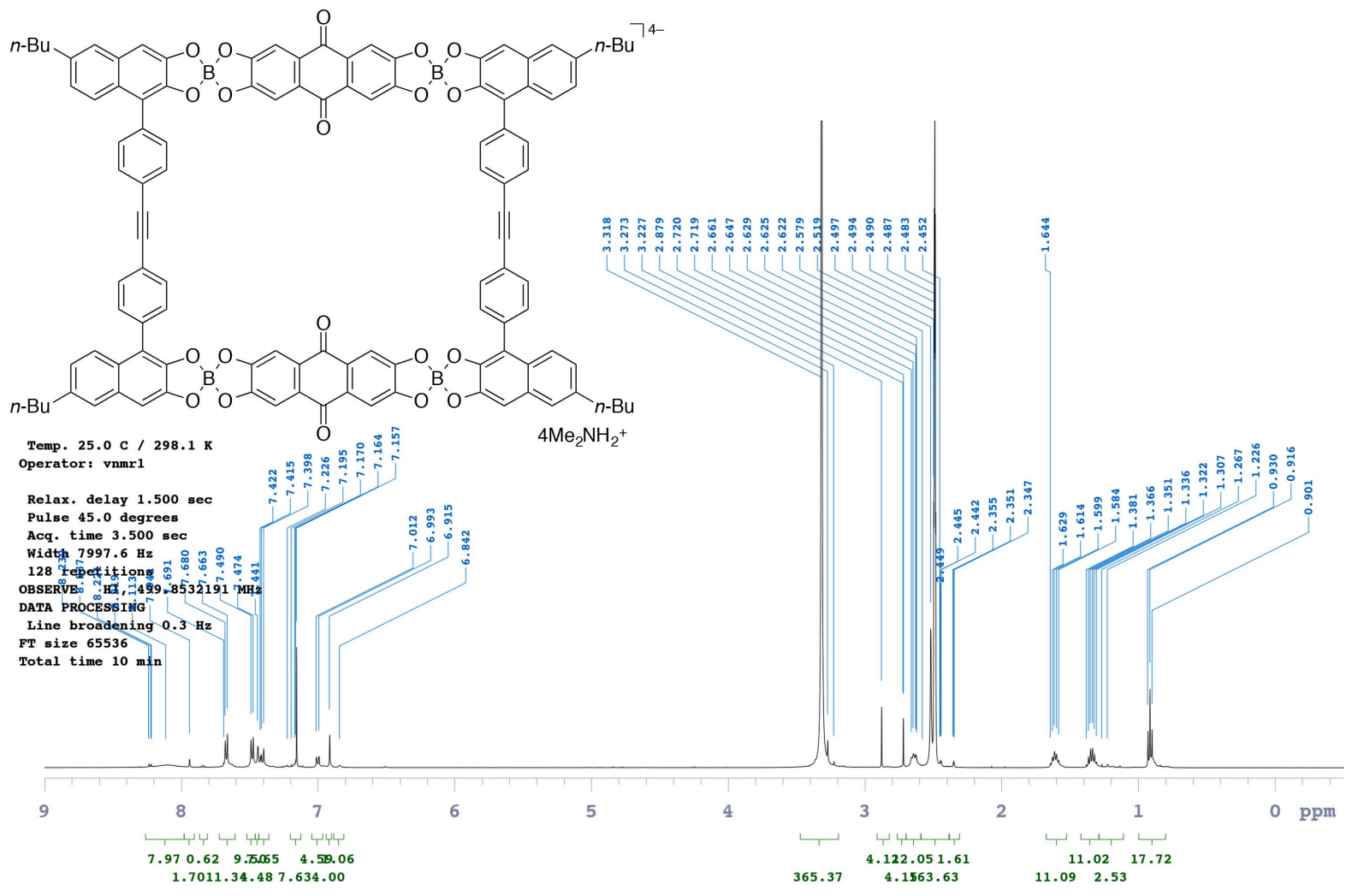

sb $\left(\mathrm{Me}_{2} \mathrm{NH}_{2}\right)_{4},{ }^{13} \mathrm{C}$ NMR $\left(126 \mathrm{MHz}\right.$, in DMSO- $\left.d_{6}, 25^{\circ} \mathrm{C}\right)$

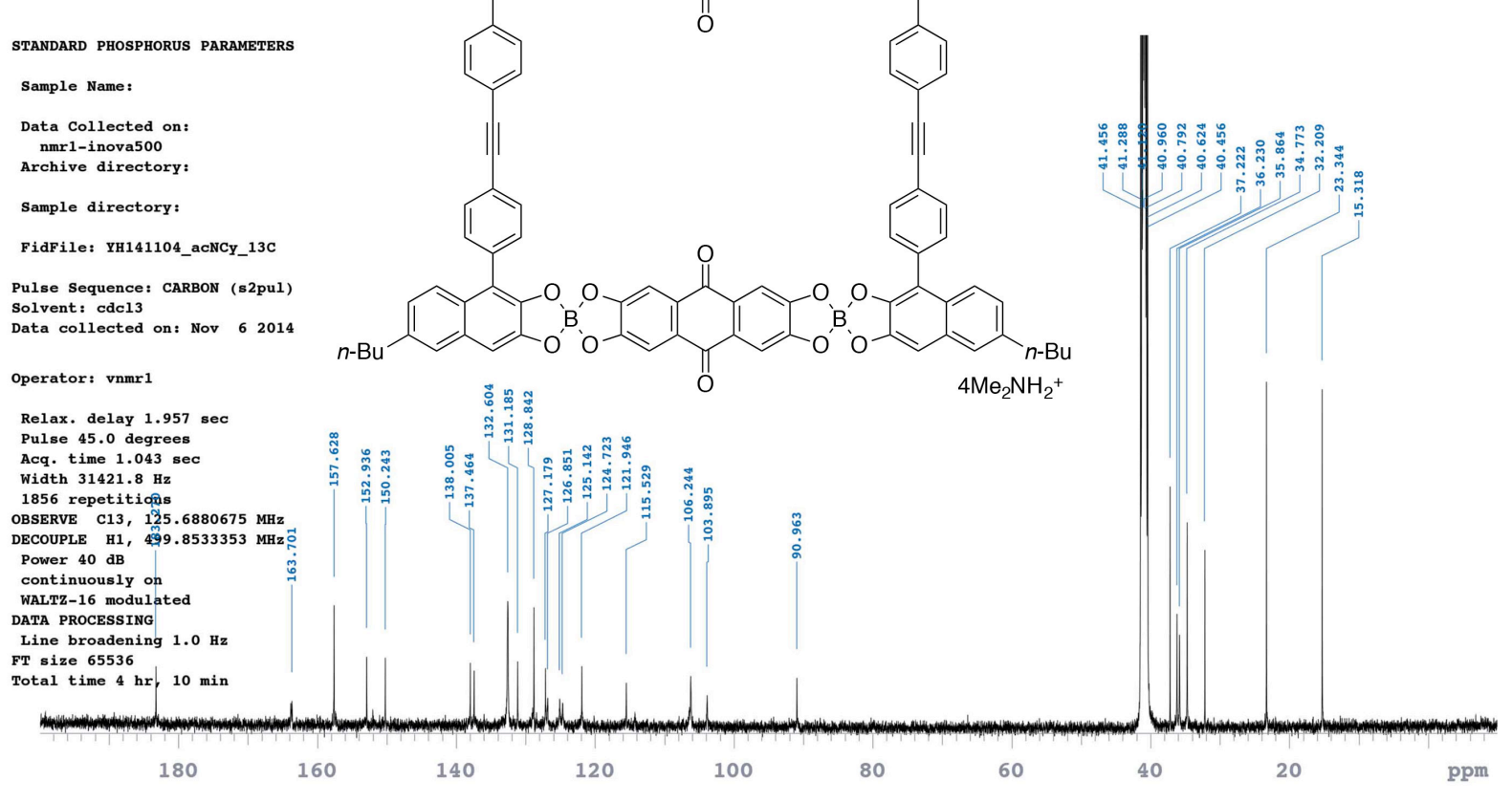

S25 
9.(TBA $)_{2},{ }^{1} \mathrm{H}$ NMR $\left(500 \mathrm{MHz}\right.$, in DMSO- $\left.d_{6}, 25^{\circ} \mathrm{C}\right)$

Sample Name:

Data Collected on: nmr1-inova500 Archive directory:

Sample directory:

FidFile: YK141224_HG10

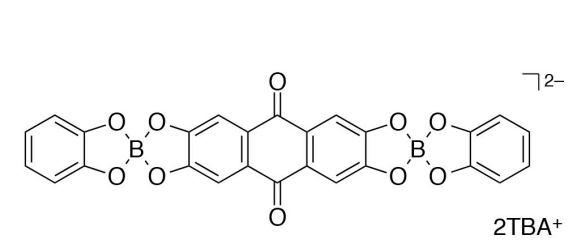

Pulse Sequence: PROTON (s2pul)

Data collected on: Dec 242014

operator: vnmr1

Relax. delay $1.500 \mathrm{sec}$ Pulse 45.0 degrees

Width $7997.6 \mathrm{~Hz}$

256 repetitions

OBSERVE H1，499.8532224 MH

DATA PROCESSING

Line broadening $0.3 \mathrm{~Hz}$

FT size 65536
Total time $21 \mathrm{~min}$

$2 \mathrm{TBA}^{+}$

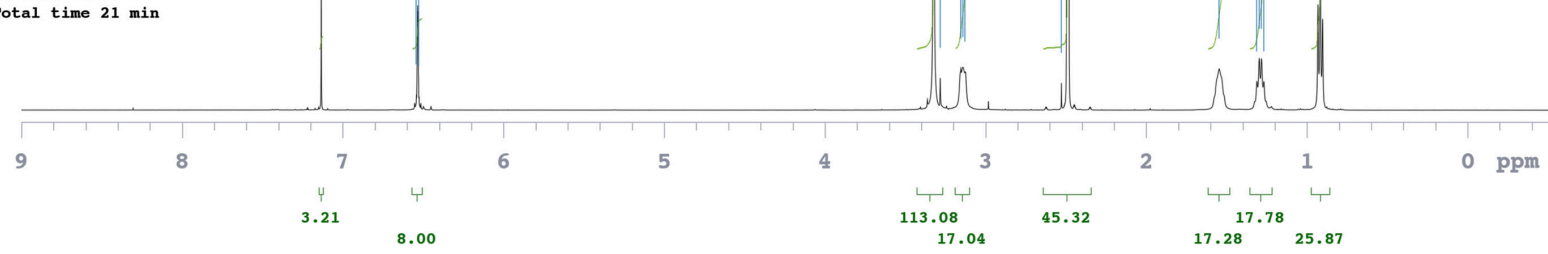

9·(TBA $)_{2},{ }^{13} \mathrm{C}$ NMR $\left(126 \mathrm{MHz}\right.$, in DMSO- $\left.d_{6}, 25^{\circ} \mathrm{C}\right)$

Sample Name:

Data Collected on:

Archive directory:

Sample directory:

FidFile: YK141224_HG10_13C

Pulse Sequence: CARBON (s2pul)

Solvent: dmso

242014

Operator: vnmr1

Relax. delay $1.957 \mathrm{sec}$

Pulse 45.0 degrees

Acq. time 1.043 sec

18000 repetition

OBSERVE C13, $125.6881879 \mathrm{MHz}$

DECOUPLE H1，499.8557096 MH

Power $40 \mathrm{~dB}$

continuously on

WALTZ-16 modulate

DATA PROCESSING

Line broadeñing $1.0 \mathrm{~Hz}$

FT size 65536

Total time $16 \mathrm{hr}, 42 \mathrm{~min}$
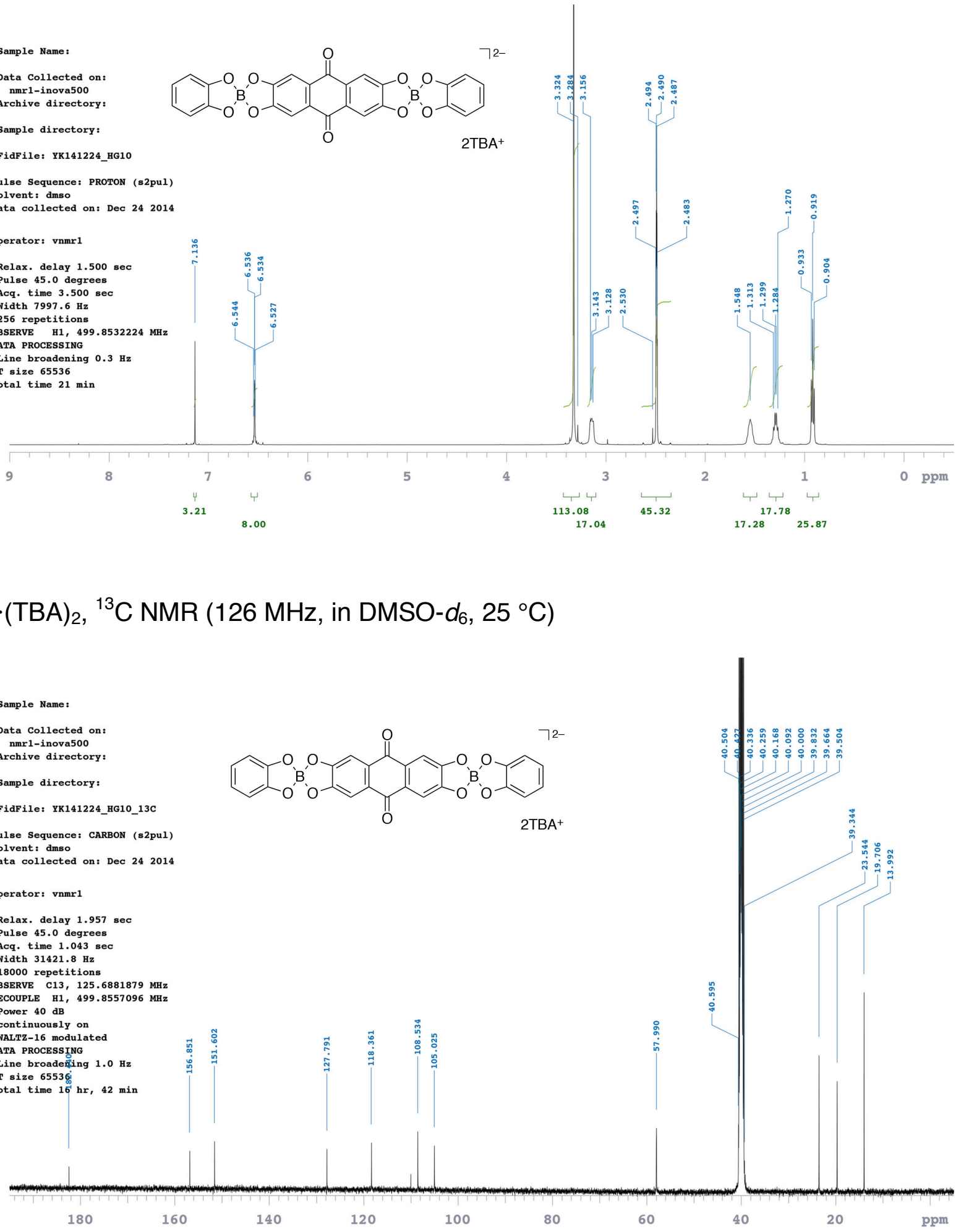


\section{${ }^{1}$ H NMR experiment (Figure 1)}

NMR experiments were performed on a Varian UNITY INOVA 500 (500 MHz) at $298 \mathrm{~K}$. In preparation of sample solutions, $1 \mathbf{a}(11.0 \mathrm{mg}, 28 \mu \mathrm{mol})$ was dissolved in DMF- $d_{7}(0.7 \mathrm{~mL})$ in a $5 \mathrm{~mm}$ quartz NMR tube (for (a)); to the solution (a) was added $\mathrm{B}(\mathrm{OH})_{3}(1.7 \mathrm{mg}, 28 \mu \mathrm{mol}$ ) and then heated at $150{ }^{\circ} \mathrm{C}$ for $24 \mathrm{~h}$ (for (b)); to the solution (b) were added 3 (7.6 mg, 28 $\mu \mathrm{mol})$ and $\mathrm{B}(\mathrm{OH})_{3}(1.7 \mathrm{mg}, 28 \mu \mathrm{mol})$, and then heated at $150{ }^{\circ} \mathrm{C}$ for $3 \mathrm{~h}$ (for (c)).

\section{Single crystal X-ray diffraction experiment (Figure 2)}

The crystal was obtained from DMF solution of $4 \mathbf{a} \cdot(\mathrm{TBA})_{4}$, that was prepared from $4 \mathbf{a} \cdot\left(\mathrm{Me}_{2} \mathrm{NH}_{2}\right)_{4}$ and excess of $\mathrm{TBABr}$, by vapor diffusion crystallization with $\mathrm{Et}_{2} \mathrm{O}$. The crystal was put on a micromount with coating Paratone oil, and placed in a cold nitrogen gas stream at $100 \mathrm{~K}$. X-ray diffraction images of the crystal were collected using a Rayonix MX225HE CCD area detector with synchrotron radiation at a wavelength of $0.80 \AA$ at the BL38B1 station of SPring-8 (Hyogo, Japan). The distance between the crystal and the detector was 55 mm. Images were processed using software HKL2000 (HKL Research). Structure solution and refinement was performed by using SHELXS-97 and SHELXL-2014/7 (Sheldrick).

The orange plate $\left(0.45 \times 0.10 \times 0.03 \mathrm{~mm}^{3}\right), \mathrm{C}_{152.5} \mathrm{H}_{199} \mathrm{~B}_{4} \mathrm{~N}_{7} \mathrm{O}_{23}, M \mathrm{r}=2541.42$; monoclinic, space group $P 2_{1} / \mathrm{c}, a=11.705(2), b=28.512(6), c=22.406(5) \AA, \alpha=90.00, \beta=98.94(3), \gamma$ $=90.00^{\circ}, V=7387(3) \AA^{3}, Z=2, D_{\text {calc }}=1.143 \mathrm{~g} \cdot \mathrm{cm}^{-3}, 6416$ unique and 5228 observed $[I>$ $2 \sigma(I)]$ reflections, 868 parameters, final $[I>2 \sigma(I)] \mathrm{R}_{1}=0.1828, w \mathrm{R}_{2}=0.4757, S=2.579$. CCDC 1045669.

\section{${ }^{1}$ H NMR experiment (Figure 4)}

NMR experiments were performed on a Varian UNITY INOVA $500(500 \mathrm{MHz})$ at $298 \mathrm{~K}$. In preparation of sample solutions, $\mathbf{5 b} \cdot\left(\mathrm{Me}_{2} \mathrm{NH}_{2}\right)_{4}(0.5 \mathrm{mM})$ (for (a)), 10 $\left(\mathrm{PF}_{6}\right)_{4}(0.5 \mathrm{mM})$ (for (b)), $11(0.5 \mathrm{mM})$ (for (c)), $0.5 \mathrm{mM} \mathrm{DMSO}-d_{6}$ solution of $\mathbf{1 0} \cdot\left(\mathrm{PF}_{6}\right)_{4}(1.5 \mathrm{mM}, 0.25 \mathrm{~mL})$ and $11(1.5 \mathrm{mM}, 0.25 \mathrm{~mL})$ and DMSO- $d_{6}(0.25 \mathrm{~mL})$ were mixed in a vial. For $(\mathrm{d}), 0.5 \mathrm{mM}$ DMSO- $d_{6}$ solution of $\mathbf{5 b} \cdot\left(\mathrm{Me}_{2} \mathrm{NH}_{2}\right)_{4}(1.5 \mathrm{mM}, 0.25 \mathrm{~mL})$ and $\mathbf{1 0} \cdot\left(\mathrm{PF}_{6}\right)_{4}(1.5 \mathrm{mM}, 0.25 \mathrm{~mL})$ and DMSO- $d_{6}(0.25 \mathrm{~mL})$ were mixed in a vial. For (e), $0.5 \mathrm{mM} \mathrm{DMSO}-d_{6}$ solution of $\mathbf{5 b} \cdot\left(\mathrm{Me}_{2} \mathrm{NH}_{2}\right)_{4}(1.5 \mathrm{mM}, 0.25 \mathrm{~mL})$ and $\mathbf{1 0} \cdot\left(\mathrm{PF}_{6}\right)_{4}(1.5 \mathrm{mM}, 0.25 \mathrm{~mL})$ and $11(1.5 \mathrm{mM}, 0.25$ $\mathrm{mL}$ ) were mixed in a vial. For (f). All solutions were transferred into a $5 \mathrm{~mm}$ NMR tube with adjusting $50 \mathrm{~mm}$ of solution height.

\section{Single crystal X-ray diffraction experiment (Figure 5)}

The crystal was obtained from DMF solution of the mixture of $\mathbf{5 a} \cdot\left(\mathrm{Me}_{2} \mathrm{NH}_{2}\right)_{4}, \mathbf{1 0} \cdot\left(\mathrm{PF}_{6}\right)_{4}$, and 11. The crystal was put on a micromount with coating Paratone oil, and placed in a cold nitrogen gas stream at $100 \mathrm{~K}$. X-ray diffraction images of the crystal were collected using a Rayonix MarMosaic225 CCD area detector with synchrotron radiation at a wavelength of 
$0.70 \AA$ at the BL26B2 station of SPring-8 (Hyogo, Japan). The distance between the crystal and the detector was $85 \mathrm{~mm}$. Images were processed using software HKL2000 (HKL Research). Structure solution and refinement was performed by using SIR-2011 and SHELXL-2013 (Sheldrick).

The brown block $\left(0.06 \times 0.04 \times 0.02 \mathrm{~mm}^{3}\right), \mathrm{C}_{186} \mathrm{H}_{168} \mathrm{~B}_{4} \mathrm{~N}_{14} \mathrm{O}_{31}, M \mathrm{r}=3138.57$; triclinic, space group $P-1, a=16.134(3) \AA, b=16.609(3) \AA, c=16.766(3) \AA, \alpha=85.82(3)^{\circ}, \beta=65.15(3)^{\circ}$, $\gamma=75.26(3)^{\circ}, V=3939.9(17) \AA^{3}, Z=1, D_{\text {calc }}=1.323 \mathrm{~g} \cdot \mathrm{cm}^{-3}, 10361$ unique and 4962 observed $[I>2 \sigma(I)]$ reflections, 1084 parameters, final $[I>2 \sigma(I)] R_{1}=0.0878, w R_{2}=0.2443$, $S=1.041$. CCDC 1045668 . 


\section{${ }^{1}$ H NMR experiment (Figure S1)}

NMR experiments were performed on a Varian UNITY INOVA 500 (500 MHz) at $298 \mathrm{~K}$. In preparation of sample solutions of (b) and (g), $2 \mathrm{mM} \mathrm{DMSO}-d_{6}$ solution of $\mathbf{4 b} \cdot\left(\mathrm{Me}_{2} \mathrm{NH}_{2}\right)_{4}$ $(0.35 \mathrm{~mL})$ and guest $(0.35 \mathrm{~mL})$ were mixed in a vial. For $(\mathrm{c}), 1.4 \mu \mathrm{L}$ of $2 \mathrm{M} \mathrm{HCl}$ aq. was added to $0.7 \mathrm{~mL}$ of (a). For (d), $0.7 \mu \mathrm{L}$ of $2 \mathrm{M} \mathrm{HCl}$ aq. was added to $0.7 \mathrm{~mL}$ of (b). All solutions were transferred into a $5 \mathrm{~mm}$ NMR tube with adjusting $50 \mathrm{~mm}$ of solution height.

a) 7

b) $7+4 \mathrm{~b} \cdot\left(\mathrm{Me}_{2} \mathrm{NH}_{2}\right)_{4}$

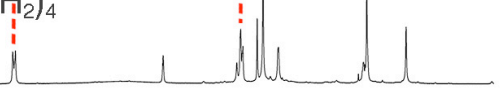

c) $7 \cdot 2 \mathrm{HCl}$

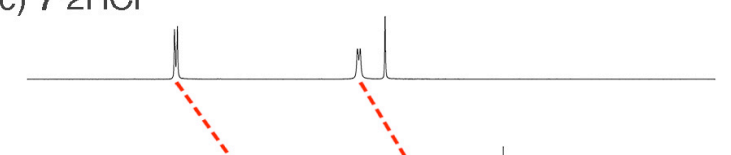

d) $7+4 b \cdot\left(\mathrm{Me}_{2} \mathrm{NH}_{2}\right)_{4}+2 \mathrm{HCl}$

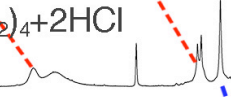

e) $4 b \cdot\left(\mathrm{Me}_{2} \mathrm{NH}_{2}\right)_{4}$

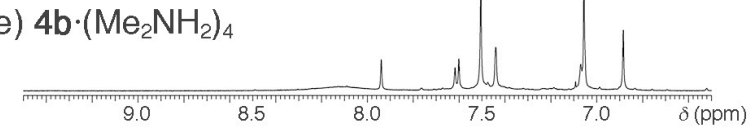

f) $8 \cdot\left(\mathrm{PF}_{6}\right)_{2}$

g) $8 \cdot\left(\mathrm{PF}_{6}\right)_{2}+4 \mathrm{~b} \cdot\left(\mathrm{Me}_{2} \mathrm{NH}_{2}\right)_{4}$

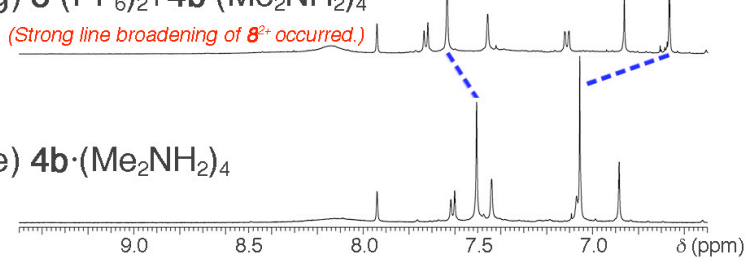

Figure S1. Partial ${ }^{1} \mathrm{H}$ NMR spectra $\left(500 \mathrm{MHz}, 25^{\circ} \mathrm{C}\right.$ in DMSO- $d_{6}$ ) of (a) 7 (2 mM), (b) 7 (1 $\mathrm{mM})$ and $\mathbf{4 b} \cdot\left(\mathrm{Me}_{2} \mathrm{NH}_{2}\right)_{4}(1 \mathrm{mM})$, (c) $7(2 \mathrm{mM})$ and $\mathrm{HCl}(4 \mathrm{mM})$, (d) 7 (1 mM), 4b $\left(\mathrm{Me}_{2} \mathrm{NH}_{2}\right)_{4}$ $(1 \mathrm{mM})$, and $\mathrm{HCl}(2 \mathrm{mM})$, (e) $4 \mathbf{b} \cdot\left(\mathrm{Me}_{2} \mathrm{NH}_{2}\right)_{4}(2 \mathrm{mM})$, (f) $\mathbf{8} \cdot\left(\mathrm{PF}_{6}\right)_{2}(2 \mathrm{mM})$, and $(\mathrm{g}) \mathbf{8} \cdot\left(\mathrm{PF}_{6}\right)_{2}(1$ $\mathrm{mM})$ and $\mathbf{4 b} \cdot\left(\mathrm{Me}_{2} \mathrm{NH}_{2}\right)_{4}(1 \mathrm{mM})$. 


\section{${ }^{1}$ H NMR experiment (Figure S2)}

NMR experiments were performed on a Varian UNITY INOVA 500 (500 MHz) at $298 \mathrm{~K}$. In preparation of sample solutions, $0.35 \mathrm{~mL}$ of $4 \mathrm{mM}$ (for (b)) or $2 \mathrm{mM}$ (for (c)) of DMSO- $d_{6}$ solution of $\mathbf{8} \cdot\left(\mathrm{PF}_{6}\right)_{2}$ was added to $0.35 \mathrm{~mL}$ of $2 \mathrm{mM} \mathrm{DMSO}-d_{6}$ solution of 9.(TBA $)_{2}$. All solutions were transferred into a $5 \mathrm{~mm}$ NMR tube with adjusting $50 \mathrm{~mm}$ of solution height.

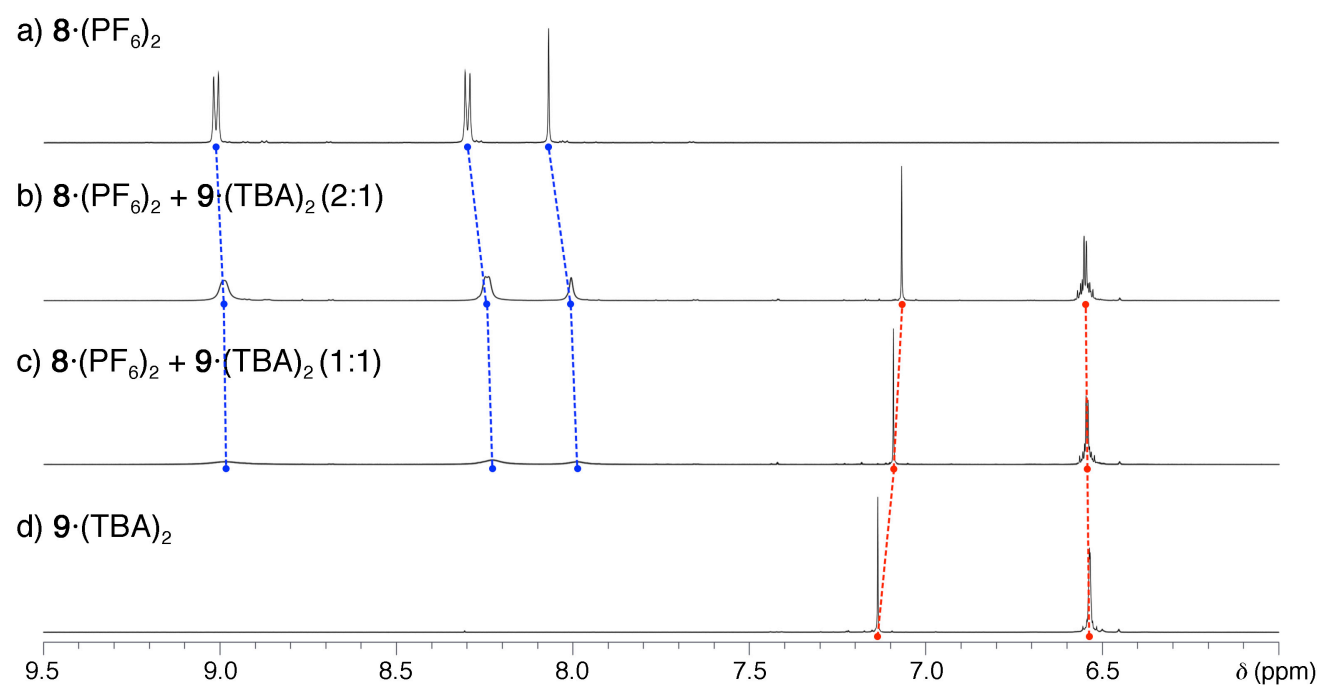

Figure S2. Partial ${ }^{1} \mathrm{H}$ NMR spectra $\left(500 \mathrm{MHz}, 25^{\circ} \mathrm{C}\right.$ in DMSO- $\left.d_{6}\right)$ of (a) 8·( $\left.\mathrm{PF}_{6}\right)_{2}(2 \mathrm{mM})$, (b) 8. $\left(\mathrm{PF}_{6}\right)_{2}(2 \mathrm{mM})$ and 9.(TBA $)_{2}(1 \mathrm{mM})$, (c) $\mathbf{8} \cdot\left(\mathrm{PF}_{6}\right)_{2}(1 \mathrm{mM})$ and $\mathbf{9} \cdot(\mathrm{TBA})_{2}(1 \mathrm{mM})$, and $(\mathrm{d})$ 9. $(\mathrm{TBA})_{2}(2 \mathrm{mM})$. 


\section{Job plot (Figure S3)}

NMR measurements were performed on a Varian UNITY INOVA $500(500 \mathrm{MHz})$ at $298 \mathrm{~K}$. Each sample solution was prepared by mixing $2 \mathrm{mM}$ DMSO- $d_{6}$ solutions of $\mathbf{4 b} \cdot\left(\mathrm{Me}_{2} \mathrm{NH}_{2}\right)_{4}$ and $\mathbf{8} \cdot\left(\mathrm{PF}_{6}\right)_{2}$ in the appropriate ratio with adjusting the total concentration of $1 \mathrm{mM}$.

a)

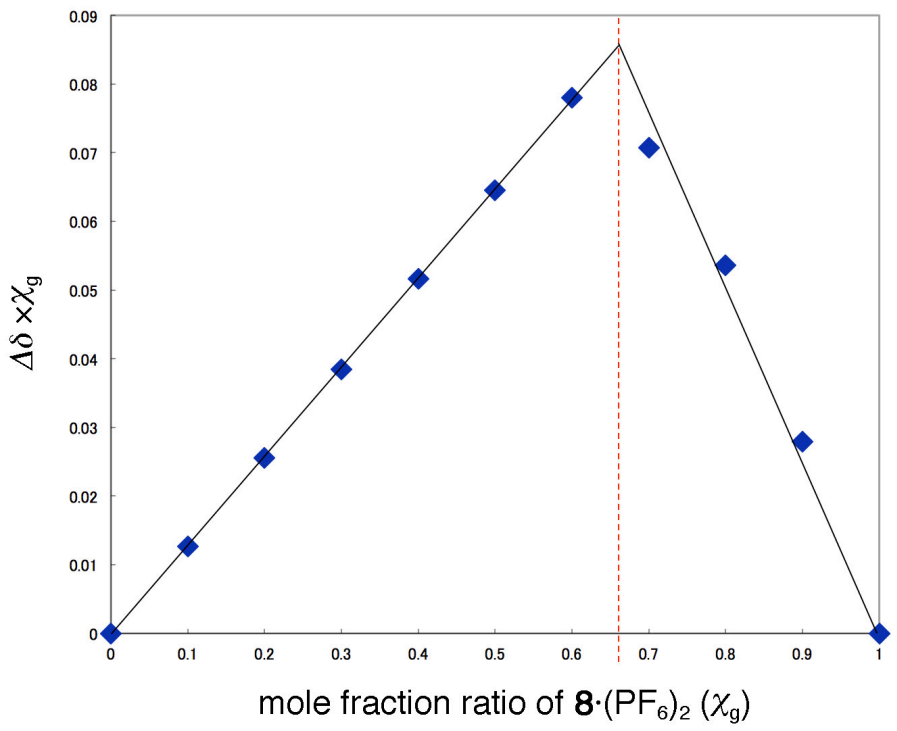

b) $4 \mathrm{~b}^{4} \cdot 8^{2+}=$

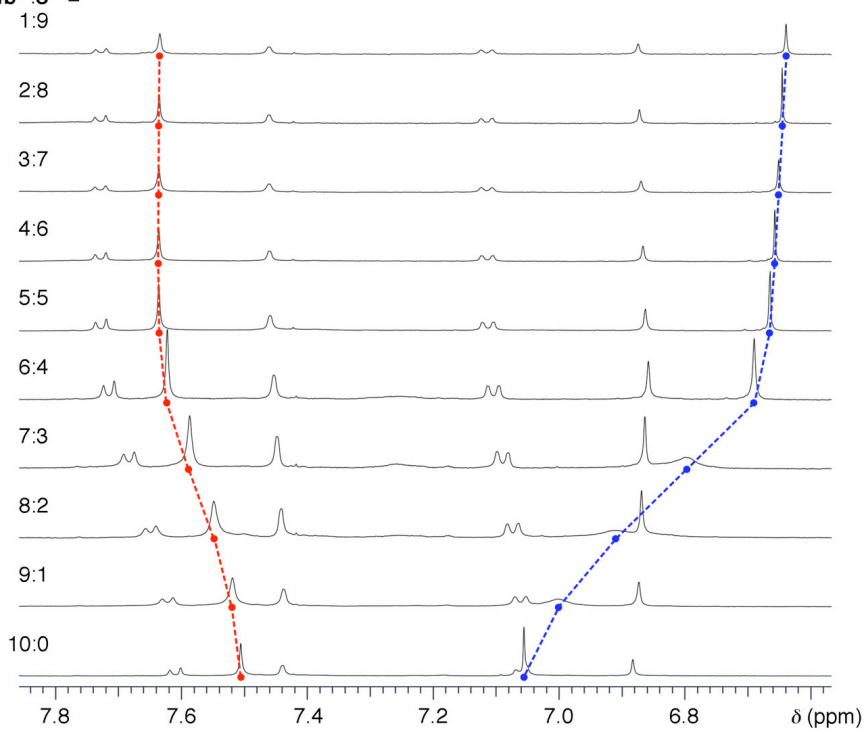

Figure S3. (a) Job plot showing the $1: 2$ binding of $\mathbf{4 b}^{4-}$ to $\mathbf{8}^{2+}$ from ${ }^{1} \mathrm{H}$ NMR signal assigned to the phenylene proton of $\mathbf{4} \mathbf{b}^{4-}$ in DMSO- $d_{6}$ at $25{ }^{\circ} \mathrm{C}$. (b) Partial ${ }^{1} \mathrm{H}$ NMR spectra $(500 \mathrm{MHz}$, $25^{\circ} \mathrm{C}$ in DMSO- $\left.d_{6}\right)$ of mixtures of $\mathbf{4 b} \cdot\left(\mathrm{Me}_{2} \mathrm{NH}_{2}\right)_{4}$ and $\mathbf{8} \cdot\left(\mathrm{PF}_{6}\right)_{2}$ in various mole fraction ratio (1 $\mathrm{mM}$ of total concentration). Signals marked by red and blue circles were assigned to the phenylene and the anthraquinone protons of $\mathbf{4} \mathbf{b}^{4-}$, respectively. 


\section{Single crystal X-ray diffraction experiment (Figure S4)}

The crystal was obtained from DMSO solution of 9.(TBA) $)_{2}$ by vapor diffusion crystallization with EtOAc. The crystal was put on a micromount with coating Paratone oil, and placed in a cold nitrogen gas stream at $100 \mathrm{~K}$. X-ray diffraction images of the crystal were collected using a Rayonix MX225HE CCD area detector with synchrotron radiation at a wavelength of $0.80 \AA$ at the BL38B1 station of SPring-8 (Hyogo, Japan). The distance between the crystal and the detector was $55 \mathrm{~mm}$. Images were processed using software HKL2000 (HKL Research). Structure solution and refinement was performed by using SHELXS-97 and SHELXL-2014/7 (Sheldrick).

The pale yellow prism $\left(0.60 \times 0.20 \times 0.20 \mathrm{~mm}^{3}\right), \mathrm{C}_{117} \mathrm{H}_{171} \mathrm{~B}_{4} \mathrm{~N}_{4} \mathrm{O}_{20}, M \mathrm{r}=1996.81$; monoclinic, space group $P 2_{1} / \mathrm{c}, a=42.193(8) \AA, b=8.3560(17) \AA, c=15.630(3) \AA, \alpha=90^{\circ}, \beta=$ $90.76(3)^{\circ}, \gamma=90^{\circ}, V=5510.1(19) \AA^{3}, Z=2, D_{\text {calc }}=1.204 \mathrm{~g} \cdot \mathrm{cm}^{-3}, 14644$ unique and 13912 observed $[I>2 \sigma(I)]$ reflections, 677 parameters, final $[I>2 \sigma(I)] R_{1}=0.0697, w R_{2}=0.1964, S$ $=1.032$. CCDC 1045670 .
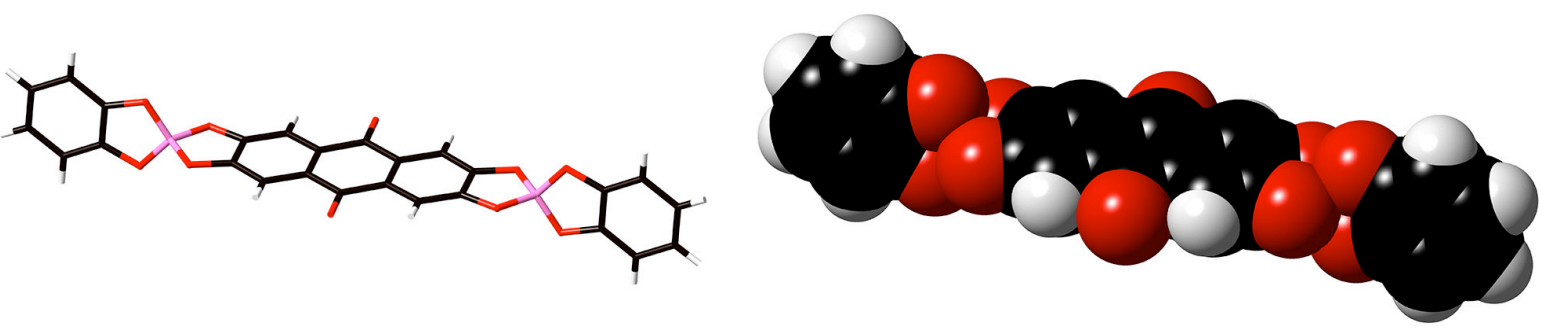

Figure S4. Crystal structure of 9.(TBA) $)_{2}$ drawn in stick (left) and space filling (right) models. Counterions $\left(\mathrm{TBA}^{+}\right)$are omitted for clarity. 


\section{Single crystal X-ray diffraction experiment (Figure S5)}

The crystal was obtained from DMF solution of the mixture of $\mathbf{1 0} \cdot\left(\mathrm{PF}_{6}\right)_{4}$ and $\mathbf{1 1}$. The crystal was put on a micromount with coating Paratone oil, and placed in a cold nitrogen gas stream at $100 \mathrm{~K}$. X-ray diffraction images of the crystal were collected using a Rayonix MarMosaic225 CCD area detector with synchrotron radiation at a wavelength of $0.70 \AA$ at the BL26B2 station of SPring-8 (Hyogo, Japan). The distance between the crystal and the detector was $85 \mathrm{~mm}$. Images were processed using software HKL2000 (HKL Research). Structure solution and refinement was performed by using SIR-2011 and SHELXL-2013 (Sheldrick).

The pale yellow prism $\left(0.10 \times 0.10 \times 0.10 \mathrm{~mm}^{3}\right), \mathrm{C}_{50} \mathrm{H}_{44} \mathrm{~F}_{24} \mathrm{~N}_{4} \mathrm{P}_{4}, M r=1280.77$; monoclinic, space group $P 2_{1} / \mathrm{n}, a=13.488(3) \AA, b=14.281(3) \AA, c=14.287(3) \AA, \alpha=90^{\circ}, \beta=$ 108.36(3) $)^{\circ}, \gamma=90^{\circ}, V=2611.9(10) \AA^{3}, Z=2, D_{\text {calc }}=1.629 \mathrm{~g} \cdot \mathrm{cm}^{-3}, 5974$ unique and 4567 observed $[I>2 \sigma(I)]$ reflections, 497 parameters, final $[I>2 \sigma(I)] R_{1}=0.0625, w R_{2}=0.1881, S$ $=1.097$. CCDC 1045666 .
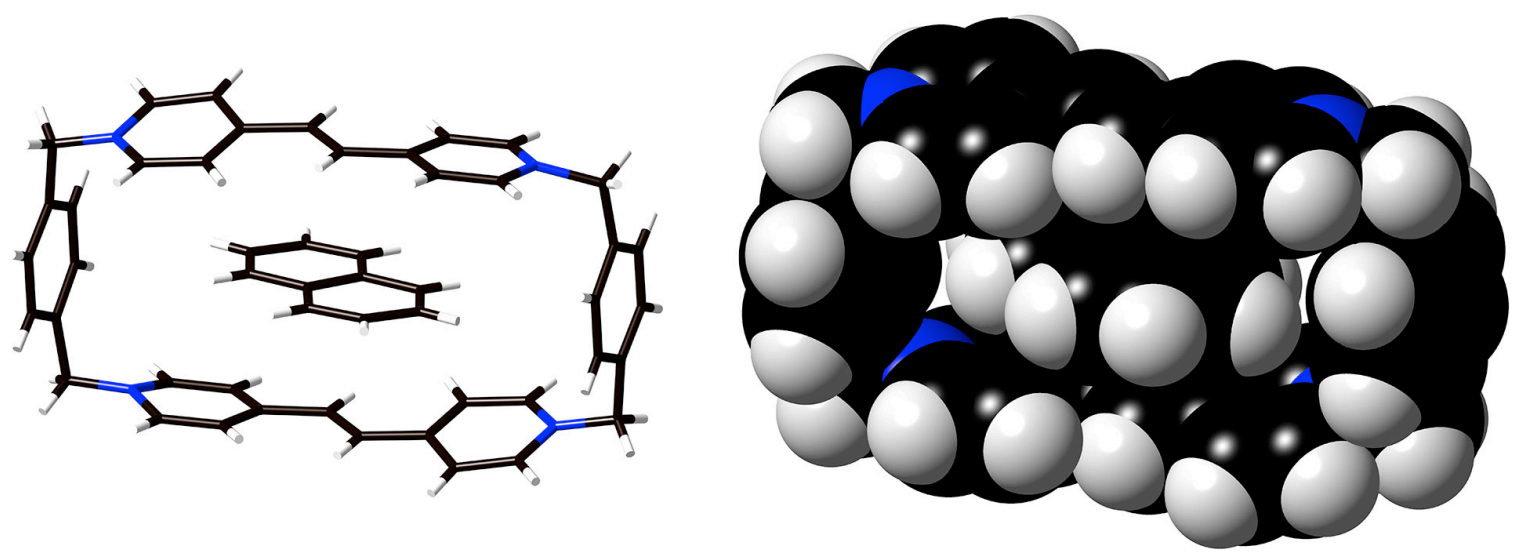

Figure S5. Crystal structure of $[11 @ 10] \cdot\left(\mathrm{PF}_{6}\right)_{4}$ drawn in stick (left) and space filling (right) models. Counterions $\left(\mathrm{PF}_{6}^{-}\right)$are omitted for clarity. 


\section{NOESY NMR experiment (Figure S6)}

NMR experiments were performed on a Varian UNITY INOVA 500 (500 MHz) at $298 \mathrm{~K}$. In preparation of sample solutions, $0.5 \mathrm{mM}$ DMSO- $d_{6}$ solution of $\mathbf{5 b} \cdot\left(\mathrm{Me}_{2} \mathrm{NH}_{2}\right)_{4}(1.5 \mathrm{mM}, 0.25$ $\mathrm{mL})$ and 10 $\left(\mathrm{PF}_{6}\right)_{4}(1.5 \mathrm{mM}, 0.25 \mathrm{~mL})$ and $11(1.5 \mathrm{mM}, 0.25 \mathrm{~mL})$ were mixed in a vial. and transferred into a $5 \mathrm{~mm}$ NMR tube with adjusting $50 \mathrm{~mm}$ of solution height.
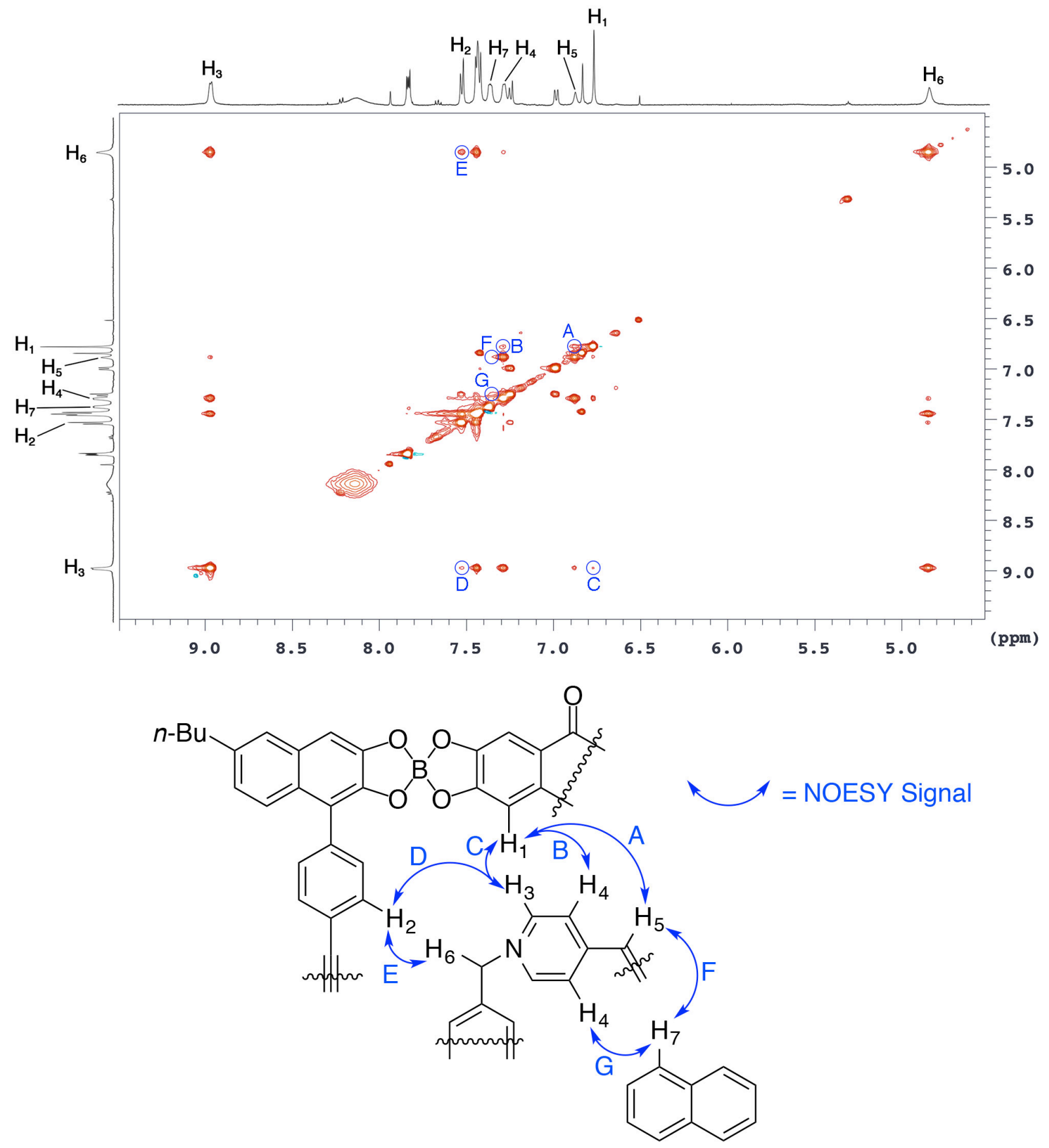

Figure S6. ${ }^{1} \mathrm{H}-{ }^{1} \mathrm{H}$ NOESY spectrum of $\mathbf{1 1} @ \mathbf{1 0} @ \mathbf{5 b}$. 


\section{CSI-MS experiment (Figure S7) ${ }^{12)}$}

Cold spray ionization MS (CSI-MS) experiments were performed on a JMS-T100LP mass spectrometer. The measurement was carried out with the following conditions;

Sample concentration: $10 \mathrm{mg} / \mathrm{L}$ in DMF/MeOH (8:2)

Ion source: CSI+

Needle voltage: $2000 \mathrm{~V}$

Ring lens voltage: $30 \mathrm{~V}$

Oriffice 1 voltage: $231 \mathrm{~V}$

Oriffice 1 temperature: $35^{\circ} \mathrm{C}$

Oriffice 2 voltage: $10 \mathrm{~V}$

Desolvating chamber temperature: $33^{\circ} \mathrm{C}$
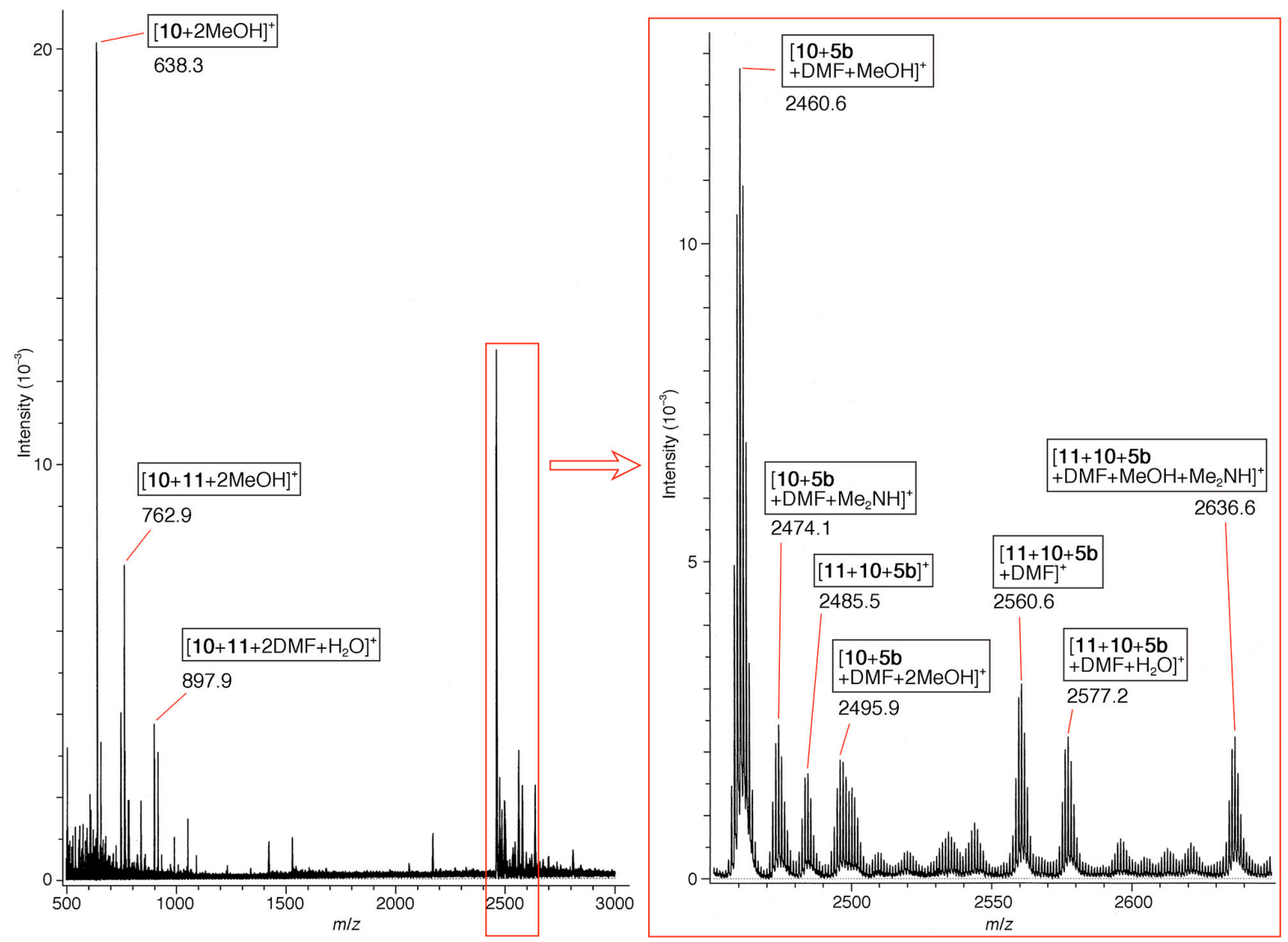

Figure S7. Positive CSI-Mass spectra of a mixture of $\mathbf{5 b} \cdot\left(\mathrm{Me}_{2} \mathrm{NH}_{2}\right)_{4}, \mathbf{1 0} \cdot\left(\mathrm{PF}_{6}\right)_{4}$, and $\mathbf{1 1}$ (in 1:1:6 molar ratio). 


\section{Single crystal X-ray diffraction experiment (Figure S8)}

The crystal was obtained from DMSO- $d_{6}$ solution of the mixture of $\mathbf{5 b} \cdot\left(\mathrm{Me}_{2} \mathrm{NH}_{2}\right)_{4}$ and 10. $\left(\mathrm{PF}_{6}\right)_{4}$ (1:1 molar ratio). The crystal was put on a micromount with coating Paratone oil, and placed in a cold nitrogen gas stream at $100 \mathrm{~K}$. X-ray diffraction images of the crystal were collected using a Rayonix MX225HE CCD area detector with synchrotron radiation at a wavelength of $0.80 \AA$ at the BL38B1 station of SPring-8 (Hyogo, Japan). The distance between the crystal and the detector was $55 \mathrm{~mm}$. Images were processed using software HKL2000 (HKL Research). Structure solution and refinement was performed by using SHELXS-97 and SHELXL-2014 (Sheldrick).

The orange prism $\left(0.20 \times 0.10 \times 0.03 \mathrm{~mm}^{3}\right), \mathrm{C}_{180} \mathrm{H}_{112} \mathrm{~B}_{4} \mathrm{D}_{84} \mathrm{~N}_{4} \mathrm{O}_{34} \mathrm{~S}_{14}, M r=3535.99$; triclinic, space group $P-1, a=16.670(3) \AA, b=17.440(4) \AA, c=19.659(4) \AA, \alpha=81.21(3)^{\circ}, \beta=$ $74.06(3)^{\circ}, \gamma=62.19(3)^{\circ}, V=4859(2) \AA^{3}, Z=1, D_{\text {calc }}=1.208 \mathrm{~g} \cdot \mathrm{cm}^{-3}, 16254$ unique and 9444 observed $[I>2 \sigma(I)]$ reflections, 1190 parameters, final $[I>2 \sigma(I)] R_{1}=0.1643, w R_{2}=0.2091$, $S=1.787$. CCDC 1045667 .
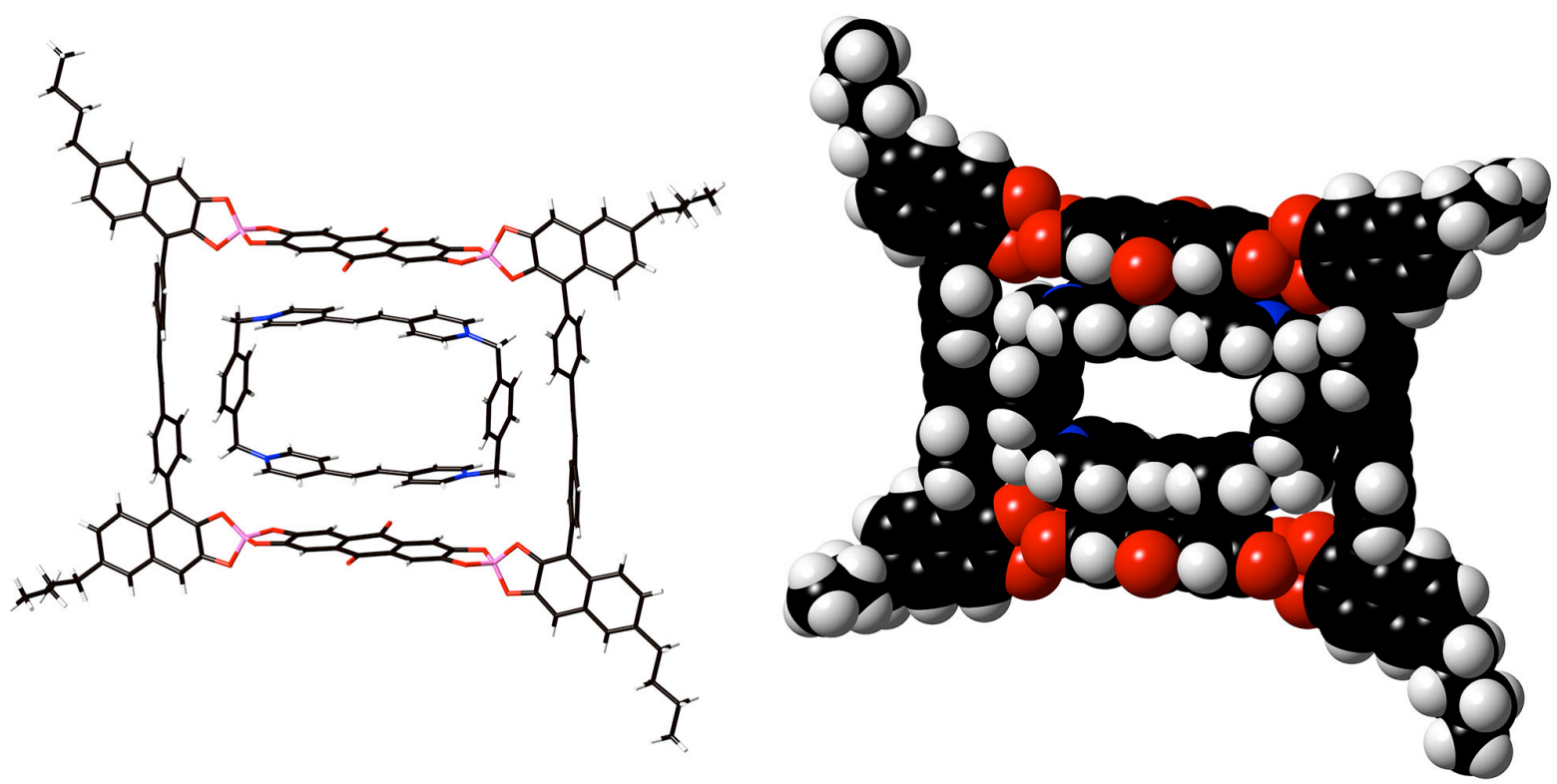

Figure S8. Crystal structure of $\mathbf{1 0} @ \mathbf{5 b}$ drawn in stick (left) and space filling (right) models. Solvent molecules (DMSO- $d_{6}$ ) are omitted for clarity. 


\section{References}

(1) Danjo, H.; Mitani, N.; Muraki, Y.; Kawahata, M.; Azumaya, I.; Yamaguchi, K.; Miyazawa, T. Chem. Asian J. 2012, 7, 1529-1532.

(2) Balaban, T. S.; Eichhöfer, A.; Krische, M. J.; Lehn, J.-M. Helv. Chim. Acta 2006, 89, 333-351.

(3) Horner, M.; Hünig, S. Liebigs Annalen Chem. 1982, 1409-1422.

(4) Ashton, P. R.; Ballardini, R.; Balzani, V.; Gandolfi, M. T.; Marquis, D. J.-F.; Pérez-Garcia, L.; Prodi, L.; Stoddart, J. F.; Venturi, M. J. Chem. Soc., Chem. Commun. 1994, 177-180.

(5) Balaban, T. S.; Eichhöfer, A.; Krische, M. J.; Lehn, J.-M. Helv. Chim. Acta 2006, 89, 333-351.

(6) Taniguchi, T.; Fukuba, T.-A.; Nakatsuka, S.; Hayase, S.; Kawatsura, M.; Uno, H.: Itoh, T. J. Org. Chem. 2008, 73, 3875-3884.

(7) Nájera, C.; Gil-Moltó, J.; Karlstöm, S. Adv. Synth. Catal. 2004, 346, 1798-1811.

(8) Iskra, J.; Stavber, S.; Zupan, M. Synthesis 2004, 1869-1873.

(9) Zhu,W.; Ma, D. Org. Lett. 2006, 8, 261-263.

(10) Ooi, T.; Kameda, M.; Maruoka, K. J. Am. Chem. Soc. 2003, 125, 5139-5151.

(11) Voisin, E.; Maris, T.; Wuest, J. D. Cryst. Growth Des. 2008, 8, 308-318.

(12) Yamaguchi, K. J. Mass Spectromet. 2003, 38, 473-490. 